\title{
Modeling of Differential Stripline in the Presence of Oblique Crossing Traces
}

\author{
by \\ Peter Bliznyuk-Kvitko, B. S.
}

A thesis submitted to the Faculty of Graduate and Postdoctoral Affairs in partial fulfillment of the requirements

for the degree of

Master of Applied Science

in

Electrical and Computer Engineering

Carleton University

Ottawa, Ontario

(C) 2020, Peter Bliznyuk-Kvitko 
The undersigned recommend to the Faculty of Graduate Studies and Research acceptance of this thesis,

"Modeling of Differential Stripline in the Presence of Oblique Crossing Traces"

submitted by Peter Bliznyuk-Kvitko (B. S.) in partial fulfillment of the requirements for the degree of Master of Applied Sciences

Thesis Supervisor
Professor Ram Achar

Carleton University, May 2020 


\section{Abstract}

Due to continually increasing operating frequencies previously neglected effects of interconnects such as delay, crosstalk and attenuation are becoming major bottlenecks in high-speed designs. Effect of crossing trace angle on signal integrity of differential edge-coupled stripline is studied in this thesis. The closed-form solutions exist for simple TEM geometries such as symmetric or asymmetric differential striplines. In the literature analytical modeling for the case of $90^{\circ}$ trace crossings is available. However, for more complicated structures, such as differential stripline with crossing trace at oblique angles, computationally expensive 3D numerical methods are often employed. In this

thesis, an efficient modeling and analysis method is presented for high-speed interconnects represented by differential asymmetrical transmission lines with oblique crossings. The method is based on dividing the structure into several sections, then using an appropriate technique for estimating the capacitance matrix of each section and subsequently evaluating the impedance matrix using the chain parameters. The thesis also investigates the implication of different angles for crossing traces on signal integrity metrics. 


\section{Acknowledgements}

I would like to show my deep appreciation to my supervisor, professor Ram Achar, who helped me finalise my thesis. I also would like to express my gratitude to professor Ihsan Erdin, the author of the paper on $90^{\circ}$ crossings, who helped me to get started on the work in this thesis. I wish to acknowledge the help provided by the technical and support staff in the Electronics department of the Carleton University.

My special thanks to my family and friends who supported and encouraged me during my studies and writing of this thesis. 


\section{Contents}

1 Introduction 12

1.1 Background and Motivation . . . . . . . . . . . 12

1.2 Contributions . . . . . . . . . . . . . . . . . . 17

1.3 Organization of the Thesis . . . . . . . . . . 18

2 Literature Survey: Multiconductor Transmission Line Anal$\begin{array}{ll}\text { ysis } & 19\end{array}$

2.1 The Transmission-Line Equations for Multiconductor Lines . . 20

2.2 The PUL Parameters of Frequency-Dependent Multiconductor Lines . . . . . . . . . . . . . . . . . . . . 25

2.3 Frequency-Domain Analysis of Multiconductor Lines . . . . . 27

2.4 Differential and Common-Mode Impedances for Lossless Case 34

3 Literature Survey: Crossing Junctions with $90^{\circ}$ Intersection 38

3.1 Introduction . . . . . . . . . . . . . . . . 38

3.2 Modeling of Asymmetric Differential Striplines Including Crossing Junction Discontinuities . . . . . . . . . . . . 39 
3.3 Analytical Relations for the PUL Parameters of Asymmetrical Stripline Structures . . . . . . . . . . . . . . . . . . . . . . . . 42

3.4 Modeling 90 Crossing Discontinuity . . . . . . . . . . . 44

3.5 Transmission Line Representation as $A B C D$ and $Z$ parameters 45

4 Proposed Method for Analysis of Differential Transmission Lines in the Presence of Oblique Crossings 48

4.1 Outline of the Proposed Method . . . . . . . . . . . . . 48

4.2 Computation of Capacitance Matrix . . . . . . . . . . . . . 54

4.2.1 Solution of Integral Equation . . . . . . . . . . . . . 54

4.2.2 Segmentation of the Geometry . . . . . . . . . . . . 56

4.2.3 Selection of the Expansion Functions . . . . . . . . . . 60

4.2.4 Selection of the Weighting Functions . . . . . . . . 60

4.2.5 Evaluation of the Excitation Matrix Terms . . . . . . . 60

4.2.6 Evaluation of the Impedance Matrix Terms . . . . . . 61

4.2.7 Evaluation of the PUL Charge . . . . . . . . . . . . . 62

4.2.8 Evaluation of the PUL Capacitance . . . . . . . . . 62

5 Numerical Results $\quad 64$

5.1 Example 1...................... 65

5.1.1 Capacitance Matrix Elements at Different Angles . . . 68

5.1.2 Differential and Common-Mode Impedances for Lossless Case at Different Angles . . . . . . . . . . . . 81

5.1.3 Computation of $Z$ Parameters . . . . . . . . . . . 86

5.2 An Investigation of Impact of Crossing Junctions at Various Angles on Signal Integrity Performance . . . . . . . . . . . . . 100 
A Matlab Code for Calculation of Per-Unit-Length Capacitance Matrices

B Matlab Code for Calculation of Characteristic Impedance for Lossless Case

C Matlab Code for Calculation of $Z$ parameters 


\section{List of Figures}

1.1 Signal Integrity Effects of Interconnects . . . . . . . . . . 13

1.2 Cross-section of a Differential Stripline Structure . . . . . . . . 15

1.3 Differential Stripline with Crossing Trace at 90 Angle . . . . 16

1.4 Differential Stripline with Crossing Trace at $45^{\circ}$ Angle . . . . 17

2.1 Return Current Spreading in Ground Plane . . . . . . . . . . 22

2.2 Definition of Even and Odd Modes of Asymmetrical Differential Stripline . . . . . . . . . . . . . . . 34

3.1 Asymmetric Coupled Stripline (ASL) Topology . . . . . . . . 40

3.2 Modeling of ASL as Two Symmetrical Striplines: (a) Modeling of Top Part of the Traces and (b) Modeling of Bottom Part of the Traces. . . . . . . . . . . . . . . . . . 41

3.3 Cross-section of the ASL in the Presence of Crossing Trace at $90^{\circ}$ Angle. . . . . . . . . . . . . . . . 41

3.4 View of the $90^{\circ}$ Crossing Trace From the Side . . . . . . . . . 44

4.1 An Illustrative Differential Stripline with Crossing Trace at $45^{\circ}$ Angle ..................... 50 
4.2 Slicing 3D Structure into $N_{s}$ Sections in XZ-plane and Approximating them with Uniform Sections. Circled (3) represents a sample section of the crossing trace and the corresponding nearest influenced region on the differential stripline is marked by entries $(2)$ and $(1) \ldots \ldots \ldots \ldots \ldots$

4.3 Cross-sectional View of One Sample Section in XY-plane: (1) and (2) Correspond to the Differential Stripline \& (3) Represents the Crossing Trace (see Figure 4.2 for additional infor-

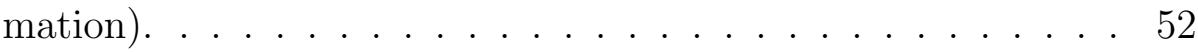

4.4 Proposed Solution for Oblique Crossing Problem . . . . . . . . 53

4.5 Segmentation of One Section of the Differential Stripline in the Presence of Crossing Trace . . . . . . . . . . . . . . . 58

4.6 Reference Segmentation Diagram with Focus on Two Segments 59

5.1 Cross-sectional View of One Sample Section in XY-plane: (1) and (2) Correspond to the Differential Stripline \& (3) Represents the Crossing Trace . . . . . . . . . . . . . . . . . . 65

5.2 Illustration of Differential Stripline with Crossing Trace at Different Angles $\left(0^{\circ}, 15^{\circ}, 30^{\circ}, 45^{\circ}\right)$ and its Segmentation into

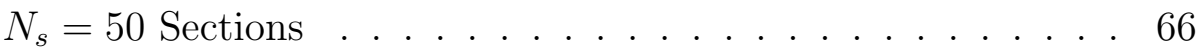

5.3 Illustration of Differential Stripline with Crossing Trace at Different Angles $\left(60^{\circ}, 75^{\circ}, 90^{\circ}\right)$ and its Segmentation into $N_{s}=50$ Sections . . . . . . . . . . . . . . . . . 67 67

5.4 Variation (of $C_{11}$ Capacitance Matrix Element) for Different Crossing Trace Angles (with $\left.N_{s}=50\right) \ldots \ldots$. . . . . 69 
5.5 Variation (of $C_{22}$ Capacitance Matrix Element) for Different Crossing Trace Angles (with $N_{s}=50$ ) . . . . . . . . . 71

5.6 Variation (of $C_{33}$ Capacitance Matrix Element) for Different Crossing Trace Angles (with $N_{s}=50$ ) . . . . . . . . . . 73

5.7 Variation (of $C_{13}$ Capacitance Matrix Element) for Different Crossing Trace Angles (with $N_{s}=50$ ) . . . . . . . . . . . . 75

5.8 Variation (of $C_{23}$ Capacitance Matrix Element) for Different Crossing Trace Angles (with $N_{s}=50$ ) . . . . . . . . . . 77

5.9 Variation (of $C_{12}$ Capacitance Matrix Element) for Different Crossing Trace Angles (with $N_{s}=50$ ) . . . . . . . . . 79

5.10 Comparative Study of Variation of Differential Impedance $Z_{d}$ for Different Crossing Trace Angles (with $N_{s}=50$ ) . . . . . 82

5.11 Comparative Study of Variation of Common-Mode Impedance $Z_{c}$ for Different Crossing Trace Angles (with $N_{s}=50$ ) . . . . 84

5.12 Experimental Setup with ASL with 1 Trace Crossing at $45^{\circ}$ Angle (Experiment 1) . . . . . . . . . . . 87

5.13 Comparison of $Z_{11}$ Parameter of the ASL with 1 Trace Crossing at $45^{\circ}$ Angle (Experiment 1) . . . . . . . . . . . 88

5.14 Comparison of $Z_{12}$ Parameter of the ASL with 1 Trace Crossing at $45^{\circ}$ Angle (Experiment 1) . . . . . . . . . . . . . . 88

5.15 Experimental Setup with ASL with 51 Trace Crossings at $45^{\circ}$ Angle (Experiment 2) . . . . . . . . . . . . . . . . . . . . . 90

5.16 Comparison of $Z_{11}$ Parameter of the ASL with 51 Trace Crossings at $45^{\circ}$ Angle (Experiment 2) . . . . . . . . . . . . 91 
5.17 Comparison of $Z_{12}$ Parameter of the ASL with 51 Trace Crossings at $45^{\circ}$ Angle (Experiment 2) . . . . . . . . . . . . 91

5.18 Experimental Setup with ASL with 51 Trace Crossings at $90^{\circ}$ Angle (Experiment 3) . . . . . . . . . . . . . . 93

5.19 Comparison of $Z_{11}$ Parameter of the ASL with 51 Trace Crossings at $90^{\circ}$ Angle (Experiment 3) . . . . . . . . . . . . . . . 94

5.20 Comparison of $Z_{12}$ Parameter of the ASL with 51 Trace Crossings at $90^{\circ}$ Angle (Experiment 3) . . . . . . . . . . . . . . . . 94

5.21 Experimental Setup for Comparison of ASLs with No Crossings, with $45^{\circ}$ Crossings and $90^{\circ}$ Crossings (Experiment 4) . . 96

5.22 Comparison of $Z_{11}$ Parameter of the ASL with 1 Trace Crossing $($ Experiment 4) . . . . . . . . . . . . . . . 97

5.23 Comparison of $Z_{12}$ Parameter of the ASL with 1 Trace Crossing (Experiment 4) . . . . . . . . . . . . . . 97

5.24 Comparison of $Z_{11}$ Parameter of the ASL with 51 Trace Crossings $($ Experiment 4) . . . . . . . . . . . . . . 98

5.25 Comparison of $Z_{12}$ Parameter of the ASL with 51 Trace Crossings $($ Experiment 4) . . . . . . . . . . . . . 98

5.26 Comparison of $Z_{11}$ Parameter of the ASL with 102 Trace Crossings (Experiment 4) . . . . . . . . . . . . . . 99

5.27 Comparison of $Z_{12}$ Parameter of the ASL with 102 Trace Crossings (Experiment 4) . . . . . . . . . . . . . . 99

5.28 ASL Cross-section Dimension Definitions . . . . . . . . . . . . 100

5.29 Eye Diagram for 7.5" ASL with No Crossings (Experiment 5) . 102 
5.30 Eye Diagram for 7.5" ASL with 102 Crossings at $90^{\circ}$ angle (Experiment 5) . . . . . . . . . . . . . . 103

5.31 Eye Diagram for 7.5" ASL with 102 Crossings at $45^{\circ}$ angle (Experiment 5) . . . . . . . . . . . . . . . . 103

5.32 Eye Diagram for 7.5" ASL with No Crossings (Experiment 8) . 107

5.33 Eye Diagram for 7.5" ASL with 102 Crossings at $90^{\circ}$ angle (Experiment 8) . . . . . . . . . . . . . . 107

5.34 Eye Diagram for 7.5" ASL with 102 Crossings at $45^{\circ}$ angle (Experiment 8) . . . . . . . . . . . . . . 108 


\section{List of Tables}

5.1 Comparison of Capacitance Matrix Element $C_{11} \ldots$. . . . 70

5.2 Comparison of Capacitance Matrix Element $C_{22} \ldots \ldots 72$

5.3 Comparison of Capacitance Matrix Element $C_{33} \ldots \ldots 74$

5.4 Comparison of Capacitance Matrix Element $C_{13} \ldots \ldots 76$

5.5 Comparison of Capacitance Matrix Element $C_{23} \ldots \ldots 78$

5.6 Comparison of Capacitance Matrix Element $C_{12} \ldots$. . . . . . 80

5.7 Comparison of Differential Impedance $Z_{d} \ldots \ldots$. . . . . . 83

5.8 Comparison of Common-Mode Impedance $Z_{c}$. . . . . . . . . 85

5.9 Cross-section Dimensions (in mils) for the Experiments 5-8 . . 101

5.10 Eye Diagram Measurements . . . . . . . . . . . . . . . 102

5.11 Eye Diagram Measurements . . . . . . . . . . . . . 104

5.12 Eye Diagram Measurements . . . . . . . . . . . . . . 105

5.13 Eye Diagram Measurements . . . . . . . . . . . . 106 


\section{Chapter 1}

\section{Introduction}

\subsection{Background and Motivation}

The rapidly increasing demand for high-speed applications coupled with significant advances in VLSI and PCB design present enormous challenges for design of next generation CAD tools for the chip, package and board levels. These challenges are diverse, such as higher transistor density, higher frequencies, sharper signal edges and lower supply voltage levels, which results in smaller timing and noise margins.

At higher frequencies and with sharpening edge rates, the interconnects can no longer be treated as simple wires, they become transmission lines propagating electromagnetic waves. In today's designs the interconnects become the major source of signal degradation due to such signal integrity effects as attenuation, delay, skew, jitter, reflections, ringing, crosstalk, skin, edge and proximity effects as well as ground bounce (see Figure 1.1). Therefore, it is increasingly important to provide accurate models of interconnects in CAD 


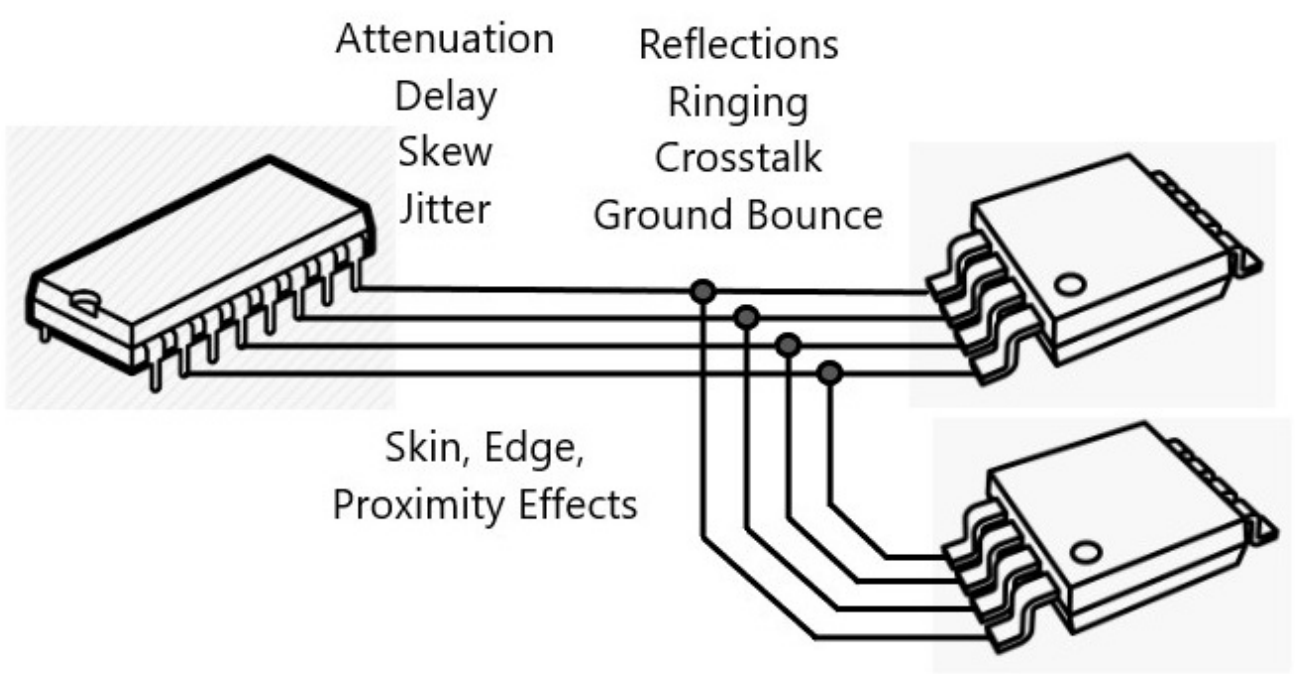

Figure 1.1: Signal Integrity Effects of Interconnects

tools and aid the designers to efficiently tackle the signal integrity and power integrity issues.

In the past, the interconnect design process heavily relied on using rules of thumb. For example, to determine if the signal will have signal integrity issues, the following rule of thumb was used: the interconnect is considered electrically long if its length is greater than the wavelength of the highest desired frequency component of the signal divided by 10. For electrically long interconnects signal integrity effects are often considered while the electrically short interconnects are treated as shorts (without considering signal integrity effects).

For simple transmission lines such as single-ended microstrip and stripline, approximate formulas were often used to calculate their parameters, giving 
them more accuracy than simple rules of thumb [4]. Per-unit length parameters (R, L, C, G), characteristic impedance, and propagation delay formulas are available for simple transmission lines from many sources. For example, the formulas for single-ended microstrip and stripline are available in [2].

For more complicated transmission lines with multiple conductors and non-uniform sections, use of formulas becomes cumbersome. Therefore, various modeling and analysis methods were developed and employed in the CAD simulators to accurately predict the performance of the transmission line. The numerical methods used in CAD simulators provide the most accuracy, but computational time becomes a trade-off [4].

Until recently, CAD signal integrity simulators took into account only major features of the interconnect, such as trace, via and pad. However, with the increasing edge rates, other design trends and features such as differential signaling, trace bends and crossing junctions start to have an effect on signal integrity and need to be accurately and efficiently modeled as well.

Differential signalling is widely used in modern designs to improve the noise related figure of merit. Striplines are often used for implementing differential signaling. Stripline is a widely-used type of transmission line structure in carrying high-speed signals on PCBs. In the stripline structure, transmission line traces are routed between reference planes (power or ground) surrounded by a dielectric material, see Figure 1.2. Modern PCBs tend to have high routing density due to large number of ICs and each IC in turn with large number of pins. Therefore, new routing layers are added to the stackup; however it increases the cost of PCBs. To reduce the stackup height it is common to include two routing layers between reference planes in a dual 


\section{Top Reference Plane}

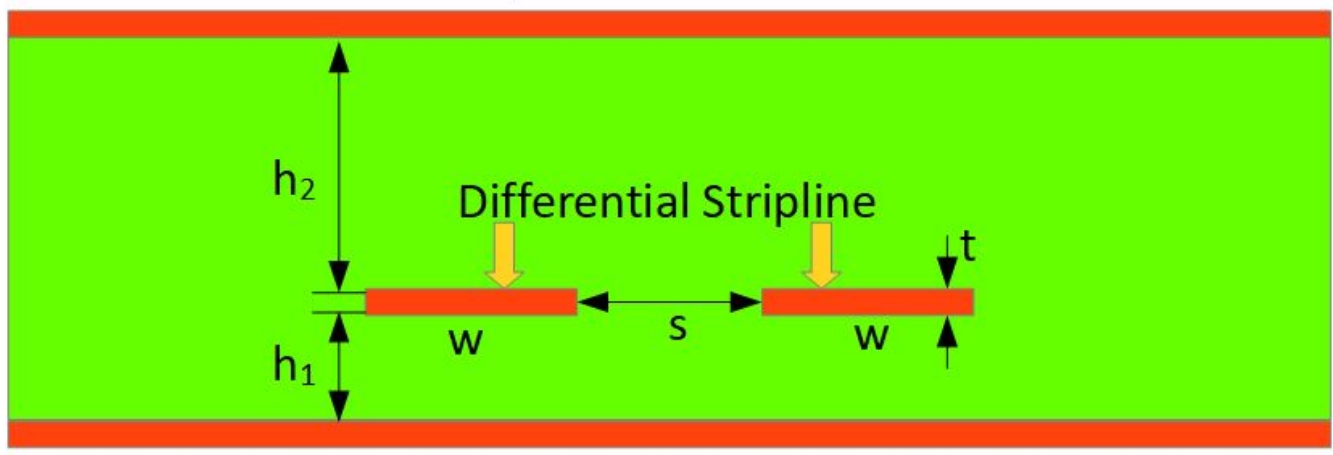

\section{Bottom Reference Plane}

Figure 1.2: Cross-section of a Differential Stripline Structure

stripline configuration, see Figure 1.3. The work in this thesis focuses on differential signaling with striplines. Differential stripline is typically routed on one of the layers of dual stripline and the crossing trace at any angle is routed on the other layer of dual stripline. For the purpose of illustration, 3D representations of the differential stripline in the presence of crossing trace at $90^{\circ}$ and $45^{\circ}$ angles are shown in Figure 1.3 and 1.4, respectively.

The effect of crossing trace junctions at $90^{\circ}$ angle on differential stripline was further modeled and studied in [6] and [7]. A structure with a dielectric layer sandwiched between two solid planes electrically behaves like a microwave cavity resonator. To minimize the coupling, tracks are often routed at $90^{\circ}$ to each other in the adjacent signal layers. This is because, mutual inductance is considerably minimized due to the $90^{\circ}$ angle between the two current directions and associated magnetic field cancellation. Also, from an electrical point of view, the perpendicular crossing gives the smallest overlap area, leading to lesser electrical field coupling and hence lower junction ca- 
pacitance. Despite the relatively low coupling between two crossing signals (that are perpendicularly routed with respect to each other), discontinuities at the crossing junctions can compromise the signal integrity of the driven lines.

In [7], the issue of crossing lines and the related discontinuities was handled in entirety, with signal integrity analysis in focus. In the proposed method, coupled asymmetrical strip lines were considered as the fundamental blocks constituting the signal path (sections with and without the impact of crossing junctions).

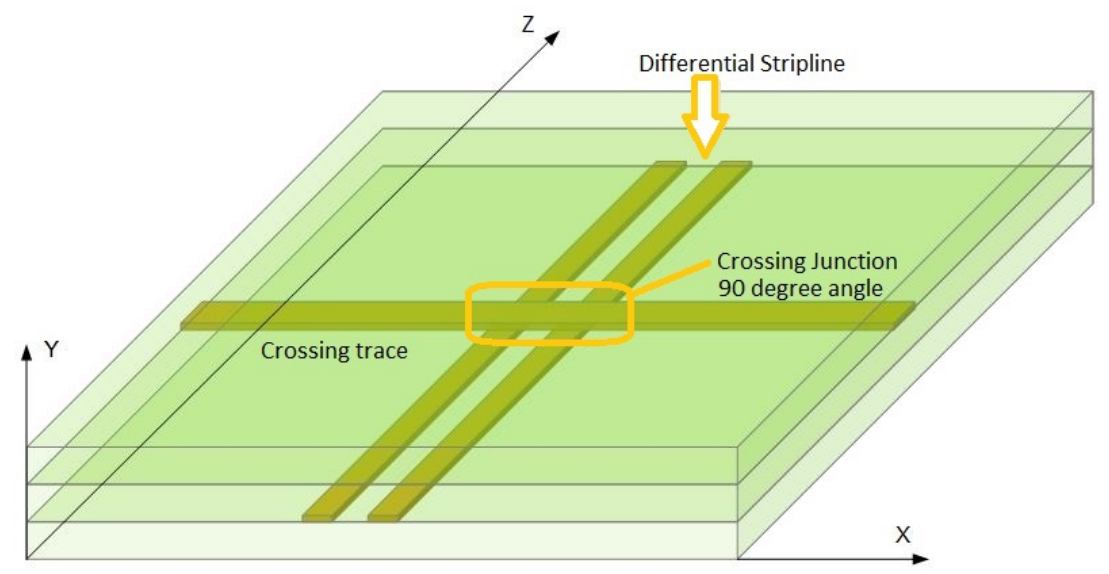

Figure 1.3: Differential Stripline with Crossing Trace at $90^{\circ}$ Angle

The work in this thesis is to further advance the research on the modeling of crossing junctions and to develop an efficient model for differential stripline in the presence of crossing traces at oblique angles $\left(0^{\circ} \leq \theta \leq 180^{\circ}\right)$ and to study the effect of crossing traces at oblique angles on the signal integrity associated with the differential striplines. The method developed in this thesis can be easily extended to model any number of crossing traces or 


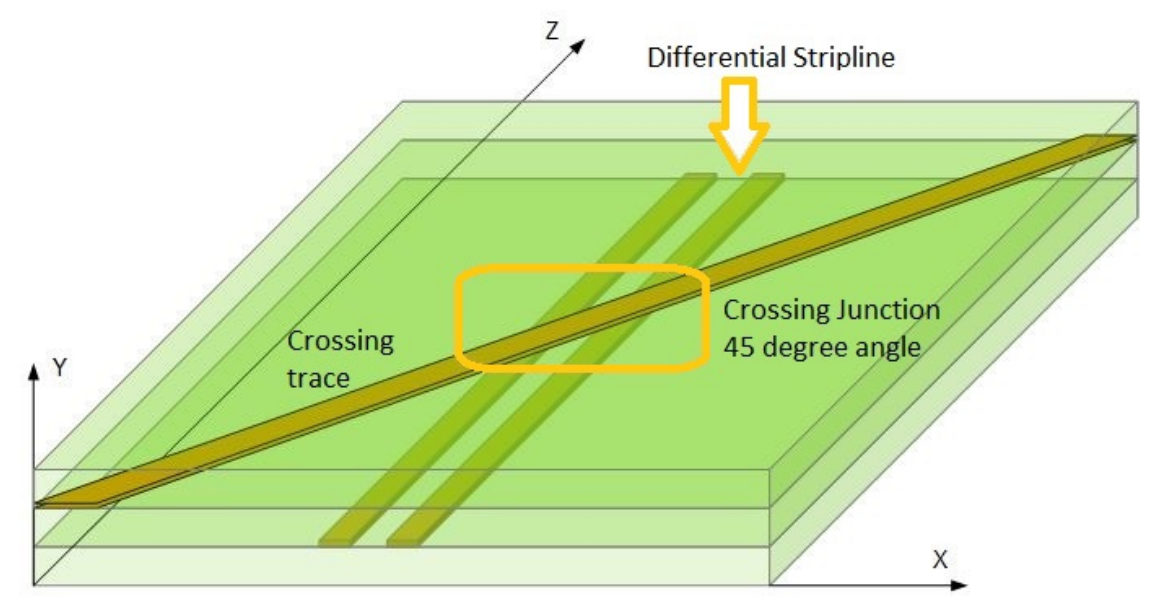

Figure 1.4: Differential Stripline with Crossing Trace at $45^{\circ}$ Angle

other conductors in the vicinity of the differential stripline.

\subsection{Contributions}

In this thesis, modeling of differential stripline with crossing trace junctions has been further advanced. Particularly,

(a) an efficient model for differential asymmetrical striplines with oblique crossing junctions has been developed.

(b) effect of the crossing trace angle on the signal integrity metrics has been investigated in detail. 


\subsection{Organization of the Thesis}

This thesis has been organized as follows. Chapter 1 provides an introduction to the topic of signal integrity as it applies to the differential stripline transmission line. Chapter 2 presents a detailed review of theory on Multiconductor Transmission Line Analysis. Chapter 3 provides a review of the previous research on $90^{\circ}$ crossings. Chapter 4 presents the proposed solution for oblique crossings. Chapter 5 presents the validating numerical results of the transmission line analysis and Chapter 6 presents the conclusions. 


\section{Chapter 2}

\section{Literature Survey:}

\section{Multiconductor Transmission Line Analysis}

Signal integrity effects in transmission lines are becoming a critical aspect of high-speed digital performance. The transmission line theory is wellestablished in the field of electrical engineering. The transmission line equations can be directly derived from Maxwell equations or from an extracted RLGC model [8]. Introductory material on signal integrity and transmission lines are available by many authors, such as [2], [3] and [4]. Widely used transmission line structures include: twisted-pair, single-ended microstrip and stripline. The relations for calculating per-unit length (PUL) parameters, characteristic impedance and propagation delay for twisted-pair, singleended microstrip and stripline are available in [2].

The analysis of both two-conductor [5] and multiconductor transmission 
lines is covered in great detail in [8]. The study of two-conductor differential stripline with crossing trace junctions at $90^{\circ}$ angle in [7] uses novel approach by treating the crossing trace at $90^{\circ}$ angle as a reference plane. Consequently, the analysis in [7] uses formulations for two-conductor transmission lines to model the differential stripline. The work presented in this thesis treats the crossing trace as a third conductor in addition to the two conductors of the differential stripline and exploits the formulations for multiconductor transmission lines to arrive at an efficient model.

This section presents the underlying theoretical background for the solution of the multiconductor transmission line. The fundamental assumption in the transmission line formulations is that the electric field intensity vector and the magnetic field intensity vector lie in a plane transverse or perpendicular to the direction of the line (the Z-axis) resulting in TEM mode of propagation.

\subsection{The Transmission-Line Equations for Mul- ticonductor Lines}

The transmission line equations for $n$-conductor line in matrix form are given below, where $n$ is the number of conductors excluding the reference plane [8]

$$
\begin{gathered}
\frac{\partial}{\partial z} \mathbf{v}(z, t)=-\mathbf{R i}(z, t)-\mathbf{L} \frac{\partial}{\partial t} \mathbf{i}(z, t) \\
\frac{\partial}{\partial z} \mathbf{i}(z, t)=-\mathbf{G} \mathbf{v}(z, t)-\mathbf{C} \frac{\partial}{\partial t} \mathbf{v}(z, t)
\end{gathered}
$$


where $\mathbf{v}(z, t)$ and $\mathbf{i}(z, t)$ are $n \times 1$ column vectors with voltages and currents of each conductor as a function of line position $z$ and time $t$

$$
\begin{array}{r}
\mathbf{v}(z, t)=\left[\begin{array}{c}
v_{1}(z, t) \\
v_{2}(z, t) \\
\ldots \\
v_{n}(z, t)
\end{array}\right] \\
\mathbf{i}(z, t)=\left[\begin{array}{c}
i_{1}(z, t) \\
i_{2}(z, t) \\
\cdots \\
i_{n}(z, t)
\end{array}\right]
\end{array}
$$

The $\mathbf{R}, \mathbf{L}, \mathbf{G}, \mathbf{C}$ are per-unit-length resistance, inductance, conductance, and capacitance matrices, respectively. The resistance matrix is described as [8]

$$
\mathbf{R}=\left[\begin{array}{cccc}
r_{1} & 0 & \ldots & 0 \\
0 & r_{2} & \ldots & 0 \\
\ldots & \ldots & \ldots & \ldots \\
0 & 0 & \ldots & r_{n}
\end{array}\right]+\left[\begin{array}{cccc}
r_{0} & r_{0} & \ldots & r_{0} \\
r_{0} & r_{0} & \ldots & r_{0} \\
\ldots & \ldots & \ldots & \ldots \\
r_{0} & r_{0} & \ldots & r_{0}
\end{array}\right]
$$

where $r_{i}$ are self-resistances of the conductors and $r_{0}$ are due to the resistance of the ground conductor. The per-unit-length resistance matrix in (2.5) adequately represents the case of reference conductors that are finite-sized such as wires. For ground conductor in the form of large ground plane, the return currents for the conductors will spread out on the ground plane beneath them as shown in Figure 2.1, possibly overlapping and having different cross-sectional areas. This will result in different ground resistances, causing 


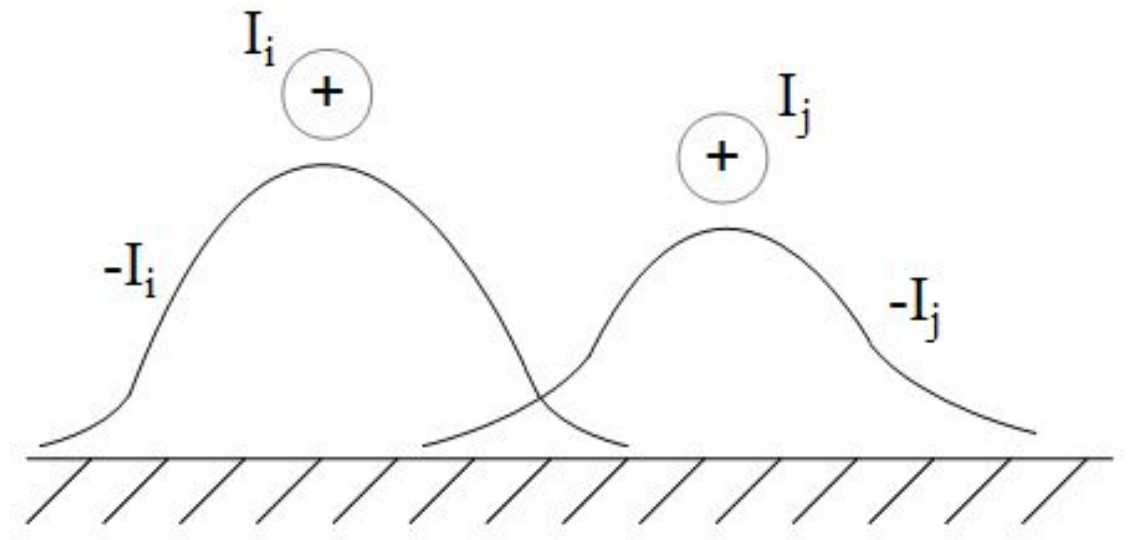

Figure 2.1: Return Current Spreading in Ground Plane

the resistance matrix to be of the form

$$
\mathbf{R}=\left[\begin{array}{cccc}
r_{1} & 0 & \ldots & 0 \\
0 & r_{2} & \ldots & 0 \\
\ldots & \ldots & \ldots & \ldots \\
0 & 0 & \ldots & r_{n}
\end{array}\right]+\left[\begin{array}{cccc}
\hat{r}_{11} & \hat{r}_{12} & \ldots & \hat{r}_{1 n} \\
\hat{r}_{12} & \hat{r}_{22} & \ldots & \hat{r}_{2 n} \\
\ldots & \ldots & \ldots & \ldots \\
\hat{r}_{1 n} & \hat{r}_{2 n} & \ldots & \hat{r}_{n n}
\end{array}\right]=\left[\begin{array}{cccc}
r_{11} & r_{12} & \ldots & r_{1 n} \\
r_{12} & r_{22} & \ldots & r_{2 n} \\
\ldots & \ldots & \ldots & \ldots \\
r_{1 n} & r_{2 n} & \ldots & r_{n n}
\end{array}\right]
$$

where $r_{i}$ are self-resistances of the conductors and $\hat{r}_{i j}$ are due to the resistance of the ground plane.

The inductance matrix is of the form:

$$
\mathbf{L}=\left[\begin{array}{cccc}
l_{11} & l_{12} & \ldots & l_{1 n} \\
l_{12} & l_{22} & \ldots & l_{2 n} \\
\ldots & \ldots & \ldots & \ldots \\
l_{1 n} & l_{2 n} & \ldots & l_{n n}
\end{array}\right]
$$

where $l_{i i}$ are self-inductances and $l_{i j}, i \neq j$ are mutual inductances. 
The conductance matrix is of the form:

$$
\mathbf{G}=\left[\begin{array}{cccc}
G_{11} & G_{12} & \ldots & G_{1 n} \\
G_{12} & G_{22} & \ldots & G_{2 n} \\
\ldots & \ldots & \ldots & \ldots \\
G_{1 n} & G_{2 n} & \ldots & G_{n n}
\end{array}\right]=\left[\begin{array}{cccc}
\sum_{k=1}^{n} g_{1 k} & -g_{12} & \ldots & -g_{1 n} \\
-g_{12} & \sum_{k=1}^{n} g_{2 k} & \ldots & -g_{2 n} \\
\ldots & \ldots & \ldots & \ldots \\
-g_{1 n} & -g_{2 n} & \ldots & \sum_{k=1}^{n} g_{n k}
\end{array}\right]
$$

where $g_{i i}$ are self-conductances, $g_{i j}, i \neq j$ are mutual conductances, $g_{i j}=$ $g_{j i}, i \neq j$ and the sums are expanded as follows

$$
\begin{aligned}
G_{11} & =\sum_{k=1}^{n} g_{1 k}=g_{11}+g_{12}+\ldots+g_{1 n} \\
G_{22} & =\sum_{k=1}^{n} g_{2 k}=g_{21}+g_{22}+\ldots+g_{2 n} \\
G_{n n} & =\sum_{k=1}^{n} g_{n k}=g_{n 1}+g_{n 2}+\ldots+g_{n n}
\end{aligned}
$$

The capacitance matrix is of the form:

$$
\mathbf{C}=\left[\begin{array}{cccc}
C_{11} & C_{12} & \ldots & C_{1 n} \\
C_{12} & C_{22} & \ldots & C_{2 n} \\
\ldots & \ldots & \ldots & \ldots \\
C_{1 n} & C_{2 n} & \ldots & C_{n n}
\end{array}\right]=\left[\begin{array}{cccc}
\sum_{k=1}^{n} c_{1 k} & -c_{12} & \ldots & -c_{1 n} \\
-c_{12} & \sum_{k=1}^{n} c_{2 k} & \ldots & -c_{2 n} \\
\ldots & \ldots & \ldots & \ldots \\
-c_{1 n} & -c_{2 n} & \ldots & \sum_{k=1}^{n} c_{n k}
\end{array}\right]
$$

where $c_{i i}$ are self-capacitances and $c_{i j}, i \neq j$ are mutual capacitances, $c_{i j}=$ $c_{j i}, i \neq j$ and the sums are expanded as follows

$$
\begin{aligned}
& C_{11}=\sum_{k=1}^{n} c_{1 k}=c_{11}+c_{12}+\ldots+c_{1 n} \\
& C_{22}=\sum_{k=1}^{n} c_{2 k}=c_{21}+c_{22}+\ldots+c_{2 n}
\end{aligned}
$$




$$
C_{n n}=\sum_{k=1}^{n} c_{n k}=c_{n 1}+c_{n 2}+\ldots+c_{n n}
$$

The total charge on the $i$ th conductor per unit length $\left(q_{i}\right)$ is given by

$$
\mathrm{Q}=\mathrm{CV}
$$

where $Q$ is the $n \times 1$ column vector of the total per-unit-length charges

$$
\mathbf{Q}=\left[\begin{array}{c}
q_{1} \\
q_{2} \\
\cdots \\
q_{n}
\end{array}\right]
$$

The equation in (2.16) can be expanded as follows:

$$
\begin{aligned}
& q_{1}=C_{11} V_{1}+C_{12} V_{2}+\ldots+C_{1 n} V_{n} \\
& q_{2}=C_{21} V_{1}+C_{22} V_{2}+\ldots+C_{2 n} V_{n} \\
& q_{n}=C_{n 1} V_{1}+C_{n 2} V_{2}+\ldots+C_{n n} V_{n}
\end{aligned}
$$

where $C_{i j}$ are elements of the capacitance matrix $\mathbf{C}$. The above equations can be written in terms of self-capacitances and mutual capacitances as follows:

$$
\begin{aligned}
& q_{1}=c_{11} V_{1}+c_{12}\left(V_{1}-V_{2}\right)+\ldots+c_{1 n}\left(V_{1}-V_{n}\right) \\
& q_{2}=c_{21}\left(V_{2}-V_{1}\right)+c_{22} V_{2}+\ldots+c_{2 n}\left(V_{2}-V_{n}\right) \\
& q_{n}=c_{n 1}\left(V_{n}-V_{1}\right)+c_{n 2}\left(V_{n}-V_{2}\right)+\ldots+c_{n n} V_{n}
\end{aligned}
$$

From these equations self-capacitance of a conductor can be defined as the coefficient of voltage on this conductor contributing to the total charge on the conductor. Mutual capacitance between two conductors can be defined as the 
coefficient of the difference of voltages between these conductors contributing to the total charge on the conductor.

The $\mathbf{R}, \mathbf{L}, \mathbf{G}, \mathbf{C}$ are generally frequency dependent, that is, they are function of frequency $\omega$ and can be written as $\mathbf{R}(\omega), \mathbf{L}(\omega), \mathbf{G}(\omega), \mathbf{C}(\omega)$. To write the MTL equations in (2.1) and (2.2) in frequency domain instead of time-domain, $\frac{\partial}{\partial t}$ is substituted with $j \omega$, which gives:

$$
\begin{gathered}
\frac{\partial}{\partial z} \mathbf{V}(z, \omega)=-\mathbf{Z}(\omega) \mathbf{I}(z, \omega) \\
\frac{\partial}{\partial z} \mathbf{I}(z, \omega)=-\mathbf{Y}(\omega) \mathbf{V}(z, \omega)
\end{gathered}
$$

where $\mathbf{Z}(\omega)$ and $\mathbf{Y}(\omega)$ are per-unit-length impedance and admittance matrices, respectively, and are given by

$$
\begin{aligned}
& \mathbf{Z}(\omega)=\mathbf{R}(\omega)+j \omega \mathbf{L}(\omega) \\
& \mathbf{Y}(\omega)=\mathbf{G}(\omega)+j \omega \mathbf{C}(\omega)
\end{aligned}
$$

\subsection{The PUL Parameters of Frequency-Dependent Multiconductor Lines}

In the resistance matrix from (2.6) it is assumed that the resistances of the reference plane $\hat{r}_{i j}$ are equal to zero, therefore, the resistance matrix $\mathbf{R}$ becomes

$$
\mathbf{R}=\left[\begin{array}{cccc}
r_{1} & 0 & \ldots & 0 \\
0 & r_{2} & \ldots & 0 \\
\ldots & \ldots & \ldots & \ldots \\
0 & 0 & \ldots & r_{n}
\end{array}\right]
$$


The conductor resistances in (2.28) can be calculated from [7] as DC and frequency-dependent components

$$
\begin{gathered}
R_{d c}^{\prime}=\frac{1}{\sigma_{d c} w t} \\
R^{\prime}(f)=\frac{1}{\eta(w+t)} \sqrt{\frac{\pi f \mu}{\sigma_{d c}}}
\end{gathered}
$$

where $w$ is the width of the conductor, $t$ is the thickness of the conductor, $\sigma_{d c}$ is dc conductivity, $f$ is the frequency, and $\eta$ is a constant such that $1<\eta<2$. The equation for the frequency-dependent component assumes that the width of the conductor $w$ is much larger than the thickness of the conductor $t$.

The per-unit-length parameters for multiconductor line can be written as [8]

$$
\begin{aligned}
& \mathbf{L C}=\mathbf{C L}=\mu \varepsilon \mathbf{1}_{\mathbf{n}} \\
& \mathbf{L G}=\mathbf{G L}=\mu \sigma \mathbf{1}_{\mathbf{n}}
\end{aligned}
$$

where $\varepsilon$ is the electric permittivity obtained as the product of relative permittivity $\varepsilon_{r}$ and permittivity of free space $\varepsilon_{0}: \varepsilon=\varepsilon_{r} \varepsilon_{0} ; \mu$ is the magnetic permeability obtained as the product of relative permeability $\mu_{r}$ and permeability of free space $\mu_{0}: \mu=\mu_{r} \mu_{0} ; \sigma$ is effective conductivity and $\mathbf{1}_{\mathbf{n}}$ is $n \times n$ identity matrix

$$
\mathbf{1}_{\mathbf{n}}=\left[\begin{array}{cccc}
1 & 0 & \ldots & 0 \\
0 & 1 & \ldots & 0 \\
\ldots & \ldots & \ldots & \ldots \\
0 & 0 & \ldots & 1
\end{array}\right]
$$

If the $\mathbf{C}$ matrix is known, the $\mathbf{L}$ matrix can be found from (2.31) as follows

$$
\mathbf{L}=\mu \varepsilon \mathbf{C}^{-1}
$$


Substituting (2.34) into (2.32) gives the equation for $\mathbf{G}$ in terms of $\mathbf{C}$

$$
\mathbf{G}=\frac{\sigma}{\varepsilon} \mathbf{C}
$$

The effective conductivity, representing conductive and polarization losses is

$$
\sigma=\omega \varepsilon \tan \delta
$$

where $\tan \delta$ is the loss tangent of the dielectric material. Substituting (2.36) into (2.35) gives expanded expression for $\mathbf{G}$

$$
\mathbf{G}=\omega \tan \delta \mathbf{C}
$$

\subsection{Frequency-Domain Analysis of Multicon- ductor Lines}

For the purpose of conciseness, omitting writing the $\omega$ in the frequency domain equations (2.24) and (2.25) we have:

$$
\begin{gathered}
\frac{\partial}{\partial z} \mathbf{V}(z)=-\mathbf{Z I}(z) \\
\frac{\partial}{\partial z} \mathbf{I}(z)=-\mathbf{Y} \mathbf{V}(z)
\end{gathered}
$$

By differentiating these equations with respect to line position $z$ and substituting (2.38) and (2.39), the following second-order differential equations are obtained

$$
\begin{aligned}
\frac{d^{2}}{d z^{2}} \mathbf{V}(z) & =\mathbf{Z Y V}(z) \\
\frac{d^{2}}{d z^{2}} \mathbf{I}(z) & =\mathbf{Y Z I}(z)
\end{aligned}
$$


where impedance $\mathbf{Z}$ and admittance $\mathbf{Y}$ matrices are given by

$$
\begin{aligned}
& \mathbf{Z}=\mathbf{R}+j \omega \mathbf{L} \\
& \mathbf{Y}=\mathbf{G}+j \omega \mathbf{C}
\end{aligned}
$$

The equations in (2.40) and (2.41) are coupled together because $\mathbf{Z Y}$ and $\mathbf{Y Z}$ are full matrices; that is, each set of voltages and currents $V_{i}(z)$ and $I_{i}(z)$ affects all the other sets of voltages and currents $V_{j}(z)$ and $I_{j}(z)$. The essential idea in the solution method is to decouple them with a similarity transformation [11]-[14]. To accomplish this, perform a change of variables in equations (2.40) and (2.41), apply the solution to the modified equations and then use change of variables to return to the original voltages and currents. The first step in the solution is to define the voltages and currents as transformations from modal voltages and currents $\left(\mathbf{V}_{m}(z)\right.$ and $\left.\mathbf{I}_{m}(z)\right)$ to actual line voltages and currents $(\mathbf{V}(z)$ and $\mathbf{I}(z))$ :

$$
\begin{gathered}
\mathbf{V}(z)=\mathbf{T}_{V} \mathbf{V}_{m}(z) \\
\mathbf{I}(z)=\mathbf{T}_{I} \mathbf{I}_{m}(z)
\end{gathered}
$$

Substituting these in the equations (2.40) and (2.41) gives

$$
\begin{aligned}
\frac{d^{2}}{d z^{2}} \mathbf{V}_{m}(z) & =\mathbf{T}_{V}^{-1} \mathbf{Z Y} \mathbf{T}_{V} \mathbf{V}_{m}(z)=\gamma^{2} \mathbf{V}_{m}(z) \\
\frac{d^{2}}{d z^{2}} \mathbf{I}_{m}(z) & =\mathbf{T}_{I}^{-1} \mathbf{Y} \mathbf{Z} \mathbf{T}_{I} \mathbf{I}_{m}(z)=\gamma^{2} \mathbf{I}_{m}(z)
\end{aligned}
$$

The objective is to decouple these second-order equations by finding $\mathbf{T}_{V}$ and $\mathbf{T}_{I}$ that simultaneously diagonalize $\mathbf{Z Y}$ and $\mathbf{Y Z}$ matrix products via similarity transformations as follows

$$
\mathbf{T}_{V}^{-1} \mathbf{Z Y} \mathbf{T}_{V}=\gamma^{2}
$$




$$
\mathbf{T}_{I}^{-1} \mathbf{Y Z T}_{I}=\gamma^{2}
$$

where $\gamma^{2}$ is an $n \times n$ diagonal matrix

$$
\gamma^{2}=\left[\begin{array}{cccc}
\gamma_{1}^{2} & 0 & \ldots & 0 \\
0 & \gamma_{2}^{2} & \ldots & 0 \\
\ldots & \ldots & \ldots & \ldots \\
0 & 0 & \ldots & \gamma_{n}^{2}
\end{array}\right]
$$

Thus, the equations with mode voltages and currents in (2.46) and (2.47) are decoupled and have the solution in the form of:

$$
\begin{gathered}
\mathbf{V}_{m}(z)=\mathbf{e}^{-\gamma z} \mathbf{V}_{m}^{+}+\mathbf{e}^{\gamma z} \mathbf{V}_{m}^{-} \\
\mathbf{I}_{m}(z)=\mathbf{e}^{-\gamma z} \mathbf{I}_{m}^{+}-\mathbf{e}^{\gamma z} \mathbf{I}_{m}^{-}
\end{gathered}
$$

where $\mathbf{V}_{m}^{ \pm}$and $\mathbf{I}_{m}^{ \pm}$are $n \times 1$ vectors of unknown voltages and currents traveling in forward and backward directions along $z$. The matrix exponentials are defined as

$$
\mathbf{e}^{ \pm \gamma z}=\left[\begin{array}{cccc}
\mathbf{e}^{ \pm \gamma_{1} z} & 0 & \ldots & 0 \\
0 & \mathbf{e}^{ \pm \gamma_{2} z} & \ldots & 0 \\
\ldots & \ldots & \ldots & \ldots \\
0 & 0 & \ldots & \mathbf{e}^{ \pm \gamma_{n} z}
\end{array}\right]
$$

Transforming back to actual line voltages and currents gives

$$
\begin{gathered}
\mathbf{V}(z)=\mathbf{T}_{V}\left(\mathbf{e}^{-\gamma z} \mathbf{V}_{m}^{+}+\mathbf{e}^{\gamma z} \mathbf{V}_{m}^{-}\right) \\
\mathbf{I}(z)=\mathbf{T}_{I}\left(\mathbf{e}^{-\gamma z} \mathbf{I}_{m}^{+}-\mathbf{e}^{\gamma z} \mathbf{I}_{m}^{-}\right)
\end{gathered}
$$


These equations contain 4 undetermined vectors $\mathbf{V}_{m}^{+}, \mathbf{V}_{m}^{-}, \mathbf{I}_{m}^{+}, \mathbf{I}_{m}^{-}$. To reduce the number of undetermined vectors to 2 , substitute (2.52) into (2.39) to give

$$
\begin{aligned}
\mathbf{V}(z) & =-\mathbf{Y}^{-1} \frac{d}{d z} \mathbf{I}(z)=\mathbf{Y}^{-1} \mathbf{T}_{I} \gamma\left(\mathbf{e}^{-\gamma z} \mathbf{I}_{m}^{+}+\mathbf{e}^{\gamma z} \mathbf{I}_{m}^{-}\right) \\
& =\mathbf{Y}^{-1} \mathbf{T}_{I} \gamma \mathbf{T}_{I}^{-1} \mathbf{T}_{I}\left(\mathbf{e}^{-\gamma z} \mathbf{I}_{m}^{+}+\mathbf{e}^{\gamma z} \mathbf{I}_{m}^{-}\right)
\end{aligned}
$$

Define the characteristic impedance matrix as

$$
\mathbf{Z}_{C}=\mathbf{Y}^{-1} \mathbf{T}_{I} \gamma \mathbf{T}_{I}^{-1}
$$

Next, the line voltages and currents are expressed with 2 vectors $\mathbf{I}_{m}^{+}$and $\mathbf{I}_{m}^{-}$

$$
\begin{gathered}
\mathbf{V}(z)=\mathbf{Z}_{C} \mathbf{T}_{I}\left(\mathbf{e}^{-\gamma z} \mathbf{I}_{m}^{+}+\mathbf{e}^{\gamma z} \mathbf{I}_{m}^{-}\right) \\
\mathbf{I}(z)=\mathbf{T}_{I}\left(\mathbf{e}^{-\gamma z} \mathbf{I}_{m}^{+}-\mathbf{e}^{\gamma z} \mathbf{I}_{m}^{-}\right)
\end{gathered}
$$

If matrix multiplication $\mathbf{Y Z}$ is performed by using (2.42), (2.43) and substitution is made by (2.31) and (2.32) the following relationship is obtained

$$
\mathbf{Y Z}=\mathbf{G R}+j \omega \mathbf{C R}+\left(j \omega \mu \sigma-\omega^{2} \mu \varepsilon\right) \mathbf{1}_{n}
$$

Substituting (2.35) into (2.61) results in

$$
\mathbf{Y Z}=\left(\frac{\sigma}{\varepsilon}+j \omega\right) \mathbf{C R}+\left(j \omega \mu \sigma-\omega^{2} \mu \varepsilon\right) \mathbf{1}_{n}
$$

From this equation it can be seen that only $\mathbf{C R}$ matrix product needs to be diagonalized as follows

$$
\mathbf{T}_{I}^{-1} \mathbf{C R T}_{I}=\Lambda^{2}
$$

where $\Lambda^{2}$ is an $n \times n$ diagonal matrix

$$
\Lambda^{2}=\left[\begin{array}{cccc}
\Lambda_{1}^{2} & 0 & \ldots & 0 \\
0 & \Lambda_{2}^{2} & \ldots & 0 \\
\ldots & \ldots & \ldots & \ldots \\
0 & 0 & \ldots & \Lambda_{n}^{2}
\end{array}\right]
$$


Next, $\mathbf{C}$ can be diagonalized

$$
\mathbf{U}^{t} \mathbf{C U}=\theta^{2}
$$

where $\theta^{2}$ is an $n \times n$ diagonal matrix

$$
\theta^{2}=\left[\begin{array}{cccc}
\theta_{1}^{2} & 0 & \ldots & 0 \\
0 & \theta_{2}^{2} & \ldots & 0 \\
\ldots & \ldots & \ldots & \ldots \\
0 & 0 & \ldots & \theta_{n}^{2}
\end{array}\right]
$$

In equation (2.65) $n$ columns of $\mathbf{U}$ are eigenvectors of $\mathbf{C}$ and $n$ values of $\theta^{2}$ are eigenvalues of $\mathbf{C}$. Next, $\mathbf{C R}$ matrix product is diagonalized as follows:

$$
\mathbf{S}^{t}\left[\theta \mathbf{U}^{t} \mathbf{R} \mathbf{U} \theta\right] \mathbf{S}=\Lambda^{2}
$$

where $n$ columns of $\mathbf{S}$ are eigenvectors of $\theta \mathbf{U}^{t} \mathbf{R} \mathbf{U} \theta$ and $n$ values of $\Lambda^{2}$ are eigenvalues of $\theta \mathbf{U}^{t} \mathbf{R} \mathbf{U} \theta$. The result shows that the desired transformations are:

$$
\begin{gathered}
\mathbf{T}_{I}=\mathbf{U} \theta \mathbf{S} \\
\mathbf{T}_{V}=\mathbf{U} \theta^{-1} \mathbf{S} \\
\mathbf{T}_{I}^{-1}=\mathbf{S}^{t} \theta^{-1} \mathbf{U}^{t}=\mathbf{T}_{V}^{t} \\
\mathbf{T}_{V}^{-1}=\mathbf{S}^{t} \theta \mathbf{U}^{t}=\mathbf{T}_{I}^{t}
\end{gathered}
$$

Therefore, the matrix product $\mathbf{Y Z}$ is diagonalized as follows from (2.49), (2.62) and (2.63)

$$
\mathbf{T}_{I}^{-1} \mathbf{Y Z T}_{I}=\left(\frac{\sigma}{\varepsilon}+j \omega\right) \Lambda^{2}+\left(j \omega \mu \sigma-\omega^{2} \mu \varepsilon\right) \mathbf{1}_{n}=\gamma^{2}
$$


From this equation the propagation constant diagonal matrix $\gamma$ is calculated as

$$
\gamma=\sqrt{\left(\frac{\sigma}{\varepsilon}+j \omega\right) \Lambda^{2}+\left(j \omega \mu \sigma-\omega^{2} \mu \varepsilon\right) \mathbf{1}_{n}}
$$

If the resistance matrix is already diagonal as indicated in (2.28), there is no need to diagonalize it as in (2.67), and the equation for the propagation constant diagonal matrix becomes

$$
\gamma=\sqrt{\left(\frac{\sigma}{\varepsilon}+j \omega\right) \mathbf{R} \theta^{2}+\left(j \omega \mu \sigma-\omega^{2} \mu \varepsilon\right) \mathbf{1}_{n}}
$$

The voltages and currents at the two ends of the transmission line are related by chain parameters as follows:

$$
\left[\begin{array}{l}
\mathbf{V}(L) \\
\mathbf{I}(L)
\end{array}\right]=\boldsymbol{\Phi}(L)\left[\begin{array}{l}
\mathbf{V}(0) \\
\mathbf{I}(0)
\end{array}\right]=\left[\begin{array}{ll}
\boldsymbol{\Phi}_{11}(L) & \boldsymbol{\Phi}_{\mathbf{1 2}}(L) \\
\boldsymbol{\Phi}_{\mathbf{2 1}}(L) & \boldsymbol{\Phi}_{\mathbf{2 2}}(L)
\end{array}\right]\left[\begin{array}{c}
\mathbf{V}(0) \\
\mathbf{I}(0)
\end{array}\right]
$$

where $L$ is length of the uniform transmission line. The chain parameters are calculated from (2.59) and (2.60) at $z=0$ and $z=L$ to result in:

$$
\begin{gathered}
\boldsymbol{\Phi}_{\mathbf{1 1}}(L)=\frac{1}{2} \mathbf{Y}^{-1} \mathbf{T}_{I}\left(\mathbf{e}^{\gamma L}+\mathbf{e}^{-\gamma L}\right) \mathbf{T}_{I}^{-1} \mathbf{Y} \\
\boldsymbol{\Phi}_{\mathbf{1 2}}(L)=-\frac{1}{2} \mathbf{Y}^{-1} \mathbf{T}_{I} \gamma\left(\mathbf{e}^{\gamma L}-\mathbf{e}^{-\gamma L}\right) \mathbf{T}_{I}^{-1} \\
\boldsymbol{\Phi}_{\mathbf{2 1}}(L)=-\frac{1}{2} \mathbf{T}_{I}\left(\mathbf{e}^{\gamma L}-\mathbf{e}^{-\gamma L}\right) \gamma^{-1} \mathbf{T}_{I}^{-1} \mathbf{Y} \\
\mathbf{\Phi}_{\mathbf{2 2}}(L)=\frac{1}{2} \mathbf{T}_{I}\left(\mathbf{e}^{\gamma L}+\mathbf{e}^{-\gamma L}\right) \mathbf{T}_{I}^{-1}
\end{gathered}
$$

The above calculations are performed for all frequencies in the range of interest. The chain parameters for individual sections of the transmission line are multiplied in appropriate order to obtain chain parameters of the whole transmission line using the following chain parameter property:

$$
\boldsymbol{\Phi}\left(L_{1}+L_{2}\right)=\boldsymbol{\Phi}\left(L_{2}\right) \boldsymbol{\Phi}\left(L_{1}\right)
$$


The multiplication for multiple sections is performed as shown below:

$$
\boldsymbol{\Phi}(L)=\boldsymbol{\Phi}_{\mathbf{N}}\left(L_{N}\right) \times \boldsymbol{\Phi}_{\mathbf{N}-\mathbf{1}}\left(L_{N-1}\right) \times \ldots \times \boldsymbol{\Phi}_{\mathbf{1}}\left(L_{1}\right)
$$

where the order of the sections of the transmission line is from 1 to $N$. The chain parameters of the whole transmission line are then transformed to impedance parameters or $Z$ parameters as follows:

$$
\begin{gathered}
\mathrm{Z}_{\mathbf{1 1}}=-\boldsymbol{\Phi}_{\mathbf{2 1}}{ }^{-1} \boldsymbol{\Phi}_{\mathbf{2 2}}=-\boldsymbol{\Phi}_{\mathbf{1 1}} \boldsymbol{\Phi}_{\mathbf{2 1}}{ }^{-1} \\
\mathrm{Z}_{\mathbf{1 2}}=-\boldsymbol{\Phi}_{\mathbf{2 1}}{ }^{-1} \\
\mathrm{Z}_{\mathbf{2 1}}=\boldsymbol{\Phi}_{\mathbf{1 2}}-\boldsymbol{\Phi}_{\mathbf{1 1}} \boldsymbol{\Phi}_{\mathbf{2 1}}{ }^{-1} \boldsymbol{\Phi}_{\mathbf{2 2}}=-\boldsymbol{\Phi}_{\mathbf{2 1}}{ }^{-1} \\
\mathrm{Z}_{\mathbf{2 2}}=-\boldsymbol{\Phi}_{\mathbf{1 1}} \boldsymbol{\Phi}_{\mathbf{2 1}}{ }^{-1}=-\boldsymbol{\Phi}_{\mathbf{2 1}}{ }^{-1} \boldsymbol{\Phi}_{\mathbf{2 2}}
\end{gathered}
$$

where the $Z$ parameters are defined in terms of voltages and currents at the near-end and far-end of the line, as follows:

$$
\left[\begin{array}{c}
\mathbf{V}(0) \\
\mathbf{V}(L)
\end{array}\right]=\mathbf{Z}(L)\left[\begin{array}{c}
\mathbf{I}(0) \\
-\mathbf{I}(L)
\end{array}\right]=\left[\begin{array}{ll}
\mathbf{Z}_{11} & \mathbf{Z}_{12} \\
\mathbf{Z}_{\mathbf{2 1}} & \mathbf{Z}_{\mathbf{2 2}}
\end{array}\right]\left[\begin{array}{c}
\mathbf{I}(0) \\
-\mathbf{I}(L)
\end{array}\right]
$$




\subsection{Differential and Common-Mode Impedances for Lossless Case}

Due to the presence of crossing trace, for evaluating self-capacitances of differential stripline traces, the structure is treated as an asymmetric stripline or as a nonsymmetrical directional coupler [16]. Let the odd and even mode single-ended impedances of the two conductors of the differential stripline (see Figure 2.2) be defined as follows:

$Z_{0 o}^{a}$ - odd-mode impedance of conductor $A$

$Z_{0 e}^{a}$ - even-mode impedance of conductor $A$

$Z_{0 o}^{b}$ - odd-mode impedance of conductor $B$

$Z_{0 e}^{b}$ - even-mode impedance of conductor $B$

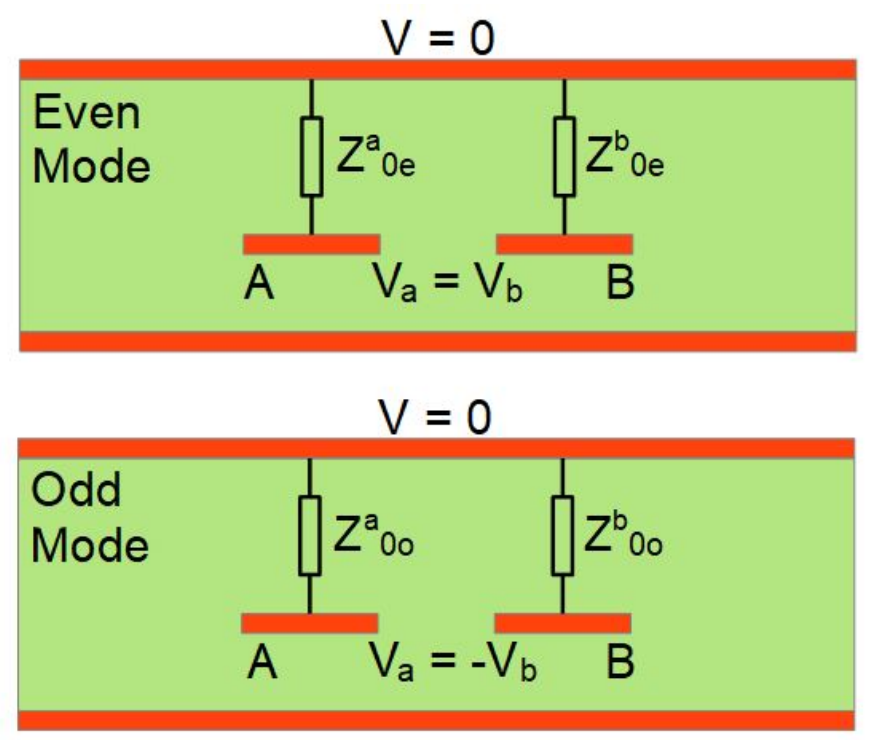

Figure 2.2: Definition of Even and Odd Modes of Asymmetrical Differential Stripline 
The odd and even mode single-ended impedances can be calculated from perunit length capacitances according to [15] for the special case of homogeneous medium using the following equations:

$$
\begin{gathered}
Z_{1}=\sqrt{\frac{z_{1}}{y_{1}}} \quad Z_{2}=\sqrt{\frac{z_{2}}{y_{2}}} \\
Z_{c}=\sqrt{Z_{1} Z_{2}} \sqrt{\frac{1+y_{m} / \sqrt{y_{1} y_{2}}}{1-y_{m} / \sqrt{y_{1} y_{2}}}} \\
Z_{\pi}=\sqrt{Z_{1} Z_{2}} \sqrt{\frac{1-y_{m} / \sqrt{y_{1} y_{2}}}{1+y_{m} / \sqrt{y_{1} y_{2}}}} \\
Z_{0 e}^{a}+Z_{0 o}^{a}=\sqrt{\frac{Z_{1}}{Z_{2}}}\left(Z_{c}+Z_{\pi}\right) \\
Z_{0 e}^{a}-Z_{0 o}^{a}=Z_{0 e}^{b}-Z_{0 o}^{b}=Z_{c}-Z_{\pi} \\
Z_{0 e}^{b}+Z_{0 o}^{b}=\sqrt{\frac{Z_{2}}{Z_{1}}}\left(Z_{c}+Z_{\pi}\right)
\end{gathered}
$$

where $y_{1}, y_{2}$ are the PUL self-admittances, and $y_{m}$ is the PUL mutual admittance of the two conductors of differential stripline in the presence of the crossing trace and are equal to the capacitance matrix elements $C_{11}, C_{22}$ and $-C_{12}$.

$$
\begin{gathered}
y_{1}=C_{11} \\
y_{2}=C_{22} \\
y_{m}=-C_{12}
\end{gathered}
$$


and $z_{1}, z_{2}$ are the PUL self-impedances of the two conductors of differential stripline in the presence of the crossing trace and are equal to the inductances $L_{11}, L_{22}$.

$$
\begin{aligned}
& z_{1}=L_{11} \\
& z_{2}=L_{22}
\end{aligned}
$$

where inductances are calculated from capacitances as follows:

$$
\begin{aligned}
& L_{11}=\mu \varepsilon C_{11}^{-1} \\
& L_{22}=\mu \varepsilon C_{22}^{-1}
\end{aligned}
$$

The differential $Z_{d}$ and common-mode $Z_{c}$ impedances can be obtained from the single-ended impedances as follows:

$$
\begin{aligned}
& Z_{d}=Z_{0 o}^{a}+Z_{0 o}^{b} \\
& Z_{c}=Z_{0 e}^{a} \| Z_{0 e}^{b}
\end{aligned}
$$

An alternative way to calculate differential and common-mode impedances is by first calculating the differential and common-mode capacitances and then calculating the impedances. First, differential and common-mode capacitances are calculated by using series and parallel capacitances rules as follows:

$$
\begin{gathered}
C_{d}=\frac{C_{11} \times C_{22}}{C_{11}+C_{22}}-\frac{C_{12}}{2} \\
C_{c}=C_{11}+C_{22}+2 \times C_{12}
\end{gathered}
$$

Next, differential and common-mode inductances are calculated as:

$$
L_{d}=\mu \varepsilon C_{d}^{-1}
$$




$$
L_{c}=\mu \varepsilon C_{c}^{-1}
$$

Subsequently, the differential and common-mode impedances are calculated as

$$
\begin{aligned}
& Z_{d}=\sqrt{\frac{L_{d}}{C_{d}}} \\
& Z_{c}=\sqrt{\frac{L_{c}}{C_{c}}}
\end{aligned}
$$




\section{Chapter 3}

\section{Literature Survey: Crossing Junctions with $90^{\circ}$ Intersection}

\subsection{Introduction}

Significant research has been previously done on the problem of striplines in the presence of crossing trace [17]-[27]. The previous work can be categorized into the following two groups.

In the first group, the problem is solved as a transmission line problem in the presence of crossing discontinuities [17]-[21]. For example, transient analysis of transmission lines (single-ended and differential) is used to solve the problem in [17] and [18]. In this work, the discontinuities are modeled as infinitely thin conducting strips. Frequency domain analysis is used to solve the problem in [19] and [20] using similar approach as in [17].

In the second group, the problem is formulated from EM equations and then solved using EM numerical methods [22], [23]. The discontinuity is 
modeled by using transverse resonance technique in [22]. The discontinuity is characterized by method of lines in [23]. All of the research work in [17]-[23] are concerned with $90^{\circ}$ angle crossings between two striplines. Coupling at oblique angles in free space of ideal, infinitely thin transmission lines is studied in [27].

\subsection{Modeling of Asymmetric Differential Striplines Including Crossing Junction Discontinu- ities}

The problem of differential stripline in the presence of crossing trace for signal integrity applications was addressed in [7]. In this approach, the entire transmission line consisting of multiple sections with and without the crossing junctions is modeled as follows:

1. An analytical method was developed to enhance the accuracy of per unit length (PUL) parameters [6] of asymmetric stripline (ASL) configurations, including the finite thickness of the conductors. DC- and frequency dependent losses are also appropriately incorporated.

2. An analytical method was proposed to evaluate the PUL parameters of the resulting ASL sections including fringing capacitances for the regions with crossing junctions.

3. A systematic and modular method was developed to combine the sections of ASL blocks constituting the signal path, in the form of cascaded 
$A B C D$ matrices.

The main approach to solving the $90^{\circ}$ crossing junction problem taken in [7] can be summarized as follows: A formula for even-mode and odd-mode PUL capacitances of ASL represented in Figure 3.1 is derived by decomposing the ASL into two symmetrical striplines shown in Figure 3.2 and combining the existing formulas for symmetrical stripline from [5].

\section{Top Reference Plane}

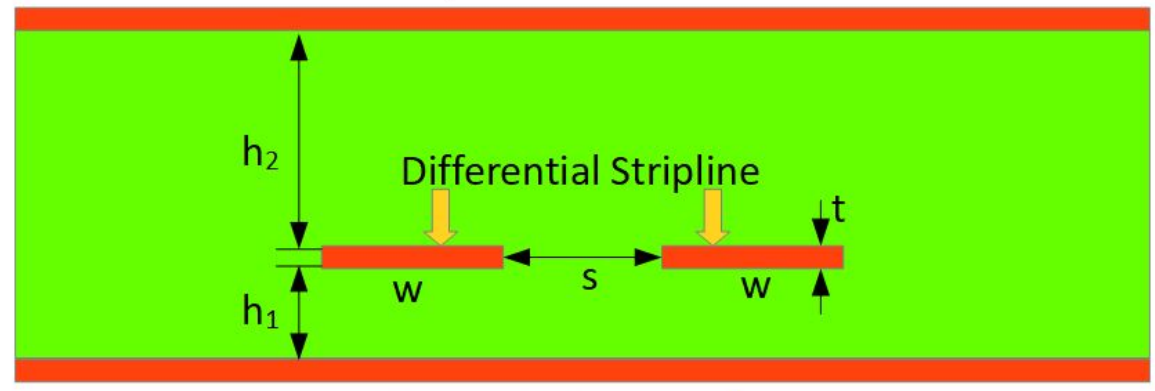

Bottom Reference Plane

Figure 3.1: Asymmetric Coupled Stripline (ASL) Topology

Next step is to solve the crossing junction topology shown in Figure 3.3 by using the derived equation for ASL and treating the crossing trace as reference plane. With solutions now available for ASL sections with and without crossing junctions, the entire transmission line can be modeled. The solution is enhanced by taking into account the trace thickness, frequencydependent losses and fringing capacitances. The $A B C D$ or chain parameters are calculated for the sections with and without the crossing traces using twoconductor analysis method described in [8] and then combined into $A B C D$ parameters of the whole structure by multiplication using the property $(2.80)$ of the chain parameters. $A B C D$ parameters of the whole structure are then 


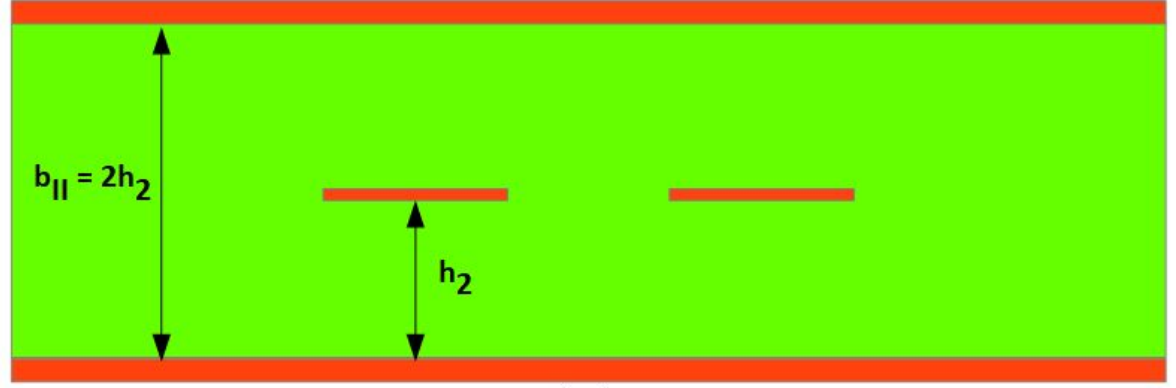

(a)

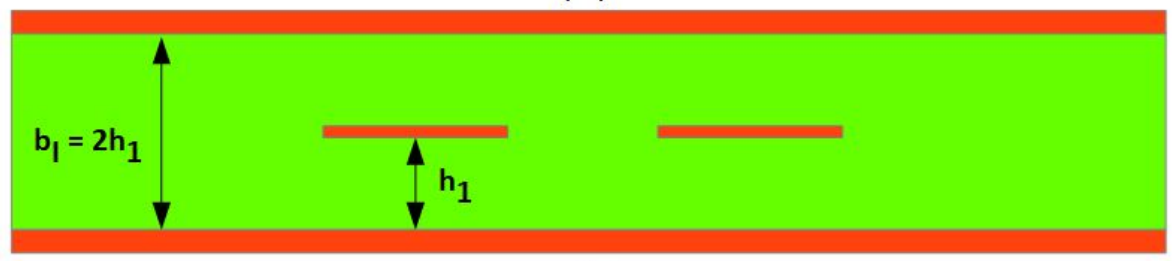

(b)

Figure 3.2: Modeling of ASL as Two Symmetrical Striplines: (a) Modeling of Top Part of the Traces and (b) Modeling of Bottom Part of the Traces.

\section{Top Reference Plane}

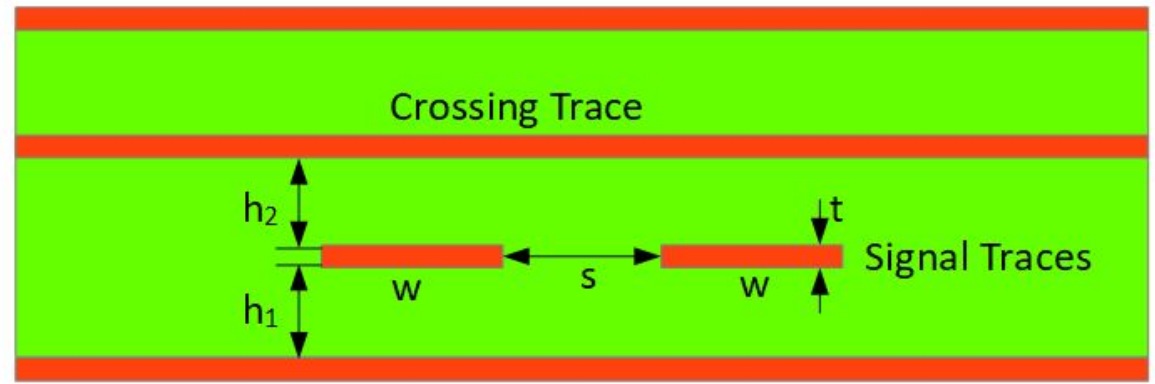

Bottom Reference Plane

Figure 3.3: Cross-section of the ASL in the Presence of Crossing Trace at $90^{\circ}$ Angle. 
transformed to $Z$ parameters to obtain a complete model of the structure to be used in simulations.

\subsection{Analytical Relations for the PUL Param- eters of Asymmetrical Stripline Structures}

By decomposing the ASL structure into two symmetrical differential striplines as shown in the previous section, the odd and even mode $\left(C_{o e}\right)$ per-unit-length capacitances of the original structure can be expressed as follows [6]

$$
C_{o e}=2 \varepsilon\left[\frac{K\left(\phi_{\text {Ioe }}\right)}{K\left(\phi_{\text {Ioe }}^{\prime}\right)}+\frac{K\left(\phi_{\text {IIoe }}\right)}{K\left(\phi_{\text {IIoe }}^{\prime}\right)}\right]
$$

where

$o$ - refers to odd mode,

$e$ - refers to even mode,

$K$ - complete elliptic integral of the first kind [5]

The modulus of the elliptic integral $\phi$ and $\phi^{\prime}$ for even and odd modes of propagation can be expressed as follows:

$$
\begin{gathered}
\phi_{i o}=\tanh \left(\frac{\pi w}{2 b_{i}}\right) \times \operatorname{coth}\left(\frac{\pi(w+s)}{2 b_{i}}\right) \\
\phi_{i e}=\tanh \left(\frac{\pi w}{2 b_{i}}\right) \times \tanh \left(\frac{\pi(w+s)}{2 b_{i}}\right) \\
\phi_{i, o e}^{\prime}=\sqrt{1-\phi_{i, o e}^{2}}
\end{gathered}
$$

where $i=I, I I$ and

$w$ - width of the conductor,

$s$ - spacing between the conductors, 
$b_{i}$ - thickness of the symmetrical striplines as defined in Figure 3.2.

The above expressions for the even and odd mode capacitances assumes infinitely thin conductors. To account for the finite thickness of the conductors, the correction factor is introduced as follows from [7]

$$
\begin{gathered}
C_{e}^{\prime}=C_{e}+2 C_{q}(t)-2 C_{q}(0) \\
C_{o}^{\prime}=C_{o}+2 \Delta C_{1}+2 C_{q}(t)-2 C_{q}(0)
\end{gathered}
$$

where

$$
\begin{gathered}
\Delta C_{1}=\varepsilon\left[\frac{t}{s}+\frac{2}{\pi}\left(1+\ln \frac{\pi}{8}\right)\right] \\
C_{q}(t)=\frac{\varepsilon}{2 \pi}\left[\frac{2}{1-\frac{t}{b}} \ln \left(1+\frac{1}{1-\frac{t}{b}}\right)-\left(\frac{1}{1-\frac{t}{b}}-1\right) \ln \left(\frac{1}{\left(1-\frac{t}{b}\right)^{2}}-1\right)\right]
\end{gathered}
$$

where $t$ is trace thickness and $b$ is thickness of the dielectric $b=h_{1}+h_{2}+t$.

The even and odd mode inductances are calculated from the corresponding capacitances similar to (2.34) for two-conductor case as

$$
L_{o e}^{\prime}=\mu \varepsilon C_{o e}^{\prime-1}
$$

The resistance is calculated as DC and frequency dependent losses according to (2.29) and (2.30). The conductance is calculated similar to (2.37) for two-conductor case as

$$
G_{o e}^{\prime}=\omega \tan \delta C_{o e}^{\prime}
$$

Both resistance and conductance are frequency-dependent, thus the accuracy of the model at high frequencies is improved. 


\subsection{Modeling $90^{\circ}$ Crossing Discontinuity}

The diagram of the ASL with $90^{\circ}$ crossing trace as viewed from the side is shown in Figure 3.4.

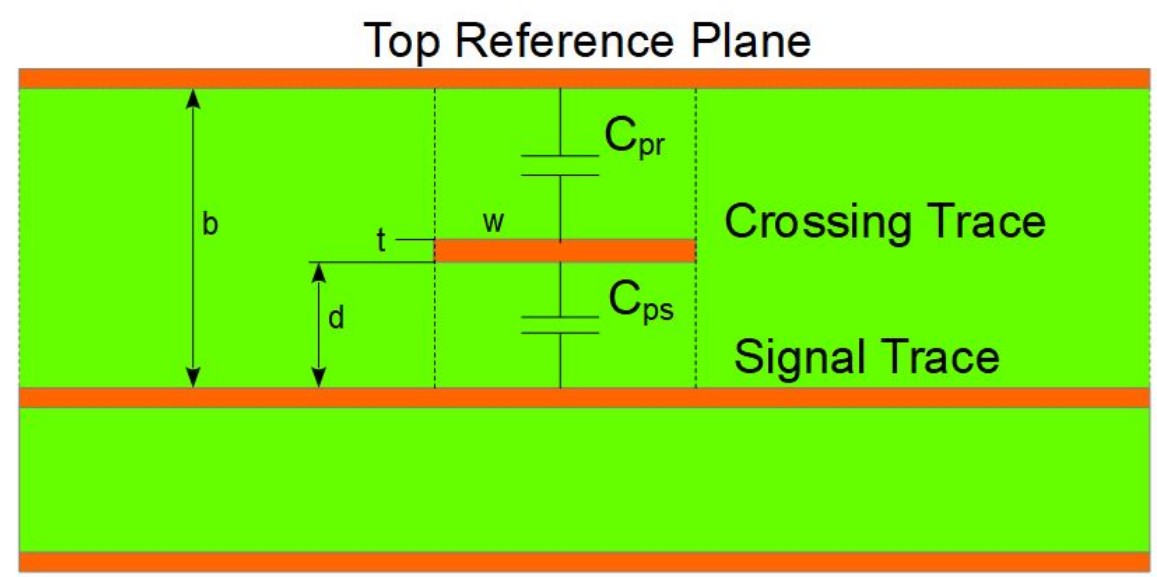

\section{Bottom Reference Plane}

Figure 3.4: View of the $90^{\circ}$ Crossing Trace From the Side

The per-unit-length capacitance of the crossing trace at the junction $C_{p p}$ can be calculated as parallel combination of the parallel-plate capacitance between the crossing trace and top reference plane $C_{p r}$ and the capacitance between the crossing trace and the signal trace $C_{p s}$.

$$
C_{p p}=C_{p r}+C_{p s}=\varepsilon\left(\frac{w}{b-t-d}+\frac{w}{d}\right)
$$

The $C_{p p}$ capacitance does not include the fringing capacitance. The total perunit-length capacitance including the parallel-plate and fringing capacitance can be calculated from [5] as

$$
C_{t}=2 \varepsilon\left(\frac{K\left(\phi_{I}\right)}{K\left(\phi_{I}{ }^{\prime}\right)}+\frac{K\left(\phi_{I I}\right)}{K\left(\phi_{I I}{ }^{\prime}\right)}\right)
$$


where

$$
\begin{gathered}
\phi_{I}=\tanh \left(\frac{\pi w}{4(b-d-t / 2)}\right) \\
\phi_{I I}=\tanh \left(\frac{\pi w}{4(d+t / 2)}\right)
\end{gathered}
$$

and $\phi_{i}^{\prime}=\sqrt{1-\phi_{i}^{2}}$; with $i=I, I I$. The finite thickness of the metal strip can be accounted for using the relations in the section 3.3. Next, the fringing capacitance can be calculated from the total capacitance and parallel-plate capacitance as

$$
C_{q}=C_{t}-C_{p p}
$$

Since $C_{q}$ only accounts for the static capacitance due to fringing fields, the correction factor for the higher order modes can be approximated by adding the modal term as

$$
B_{q}=2 \pi f C_{q}+A
$$

where $A$ is function of the wave number and the eigenvalues of the system given in [7].

\subsection{Transmission Line Representation as $A B C D$ and $Z$ parameters}

The shunt admittance of the crossing trace section can be expressed as

$$
Y_{x, o e}=s w_{x} C_{x, o e}+j 2 w B_{q}
$$

The series impedance of the crossing trace section is

$$
Z_{x, o e}=s w_{x} L_{x, o e}
$$


where $s=j 2 \pi f, f$ is the frequency, $w$ is the width of the stripline conductor, $w_{x}$ is the width of the crossing trace and $C_{x, o e}$ and $L_{x, \text { oe }}$ are per-unit-length parameters calculated using the equations for the ASL described in section 3.3. The $A B C D$ parameters of the crossing trace junction can be expressed from the admittance and impedance as follows

$$
\boldsymbol{\Psi}_{\mathbf{x}, \mathbf{o e}}=\left[\begin{array}{cc}
1+Z_{x, o e} Y_{x, o e} & Z_{x, o e} \\
Y_{x, o e} & 1
\end{array}\right]
$$

The $A B C D$ parameters of the $n$th transmission line section without the crossing trace can be obtained from [8] as

$$
\boldsymbol{\Psi}_{\mathbf{n}, \mathbf{o e}}=\left[\begin{array}{cc}
\cosh \left(\gamma_{o e} L_{n}\right) & -\sinh \left(\gamma_{o e} L_{n}\right) Z_{c, o e} \\
-\sinh \left(\gamma_{o e} L_{n}\right) Z_{c, o e}^{-1} & \cosh \left(\gamma_{o e} L_{n}\right)
\end{array}\right]
$$

where

$L_{n}$ is the length of the $n$th section,

$\gamma_{o e}$ is propagation constant for odd and even modes,

$Z_{c, o e}$ is characteristic impedance for odd and even modes

The propagation constant and characteristic impedance can be obtained as follows

$$
\begin{gathered}
\gamma_{o e}=\sqrt{\left(R_{o e}+j \omega L_{o e}\right)\left(G_{o e}+j \omega C_{o e}\right)} \\
Z_{c, o e}=\sqrt{\frac{R_{o e}+j \omega L_{o e}}{G_{o e}+j \omega C_{o e}}}
\end{gathered}
$$

By multiplying matrices in (3.19) and (3.20), the $A B C D$ parameters of the total transmission line can be computed as follows

$$
\Psi_{\mathrm{oe}}=\left[\begin{array}{cc}
A_{o e} & B_{o e} \\
C_{o e} & D_{o e}
\end{array}\right]=\boldsymbol{\Psi}_{1, \mathrm{oe}} \boldsymbol{\Psi}_{\mathbf{x}_{1}, \mathbf{o e}} \boldsymbol{\Psi}_{2, \mathrm{oe}} \boldsymbol{\Psi}_{\mathbf{x}_{2}, \mathbf{o e}} \ldots \Psi_{\mathbf{n}, \mathbf{o e}} \Psi_{\mathbf{x}_{\mathbf{n}}, \mathbf{o e}}
$$


The even and odd $A B C D$ matrices can be transformed into a Z-parameter matrix using equations in [7]. The Z-parameter matrix can be used in signal and power integrity circuit simulators to analyze the performance of the transmission line.

Method in [7] also investigated the effect of discontinuities of crossing junctions at $90^{\circ}$ on signal integrity in PCIe Gen III signaling standard using the differential pair topology with and without crossing junctions. 


\section{Chapter 4}

\section{Proposed Method for Analysis}

of Differential Transmission

\section{Lines in the Presence of}

\section{Oblique Crossings}

In this chapter, an efficient method is presented for calculation of impedance matrix $(\mathbf{Z})$ of the differential trace in the presence of oblique crossings. The method involves combining the analytical and EM methods, where appropriate, to arrive at the final solution.

\subsection{Outline of the Proposed Method}

For the purpose of illustration, an asymmetrical differential stripline in the presence of an oblique crossing trace (at $45^{\circ}$ angle) is shown in Figure 4.1. It 
is a 3D structure that is non-uniform in the direction of signal propagation along the Z-axis. The approach taken in this thesis to model this 3D structure is outlined as follows:

1. Partition the Structure into $N_{s}$ Non-uniform Sections. Divide the 3D non-uniform structure into $N_{s}$ number of non-uniform sections by slicing the 3D structure with uniform spacing in perpendicular direction to the signal propagation as shown in Figure 4.2 (which shows an illustrative case of segments with $N_{s}=50$ ).

2. Modeling of Each Non-uniform Section. Approximate each of the resulting non-uniform sections by uniform transmission line sections with three conductors (two conductors of the differential stripline and one conductor for the crossing trace) and two reference planes. An example cross-section of such uniform section is shown in Figure 4.3 (which corresponds to the circled area in Figure 4.2).

3. Computation of Capacitance Matrix. Calculate the Capacitance Matrix for each uniform section by using Method of Moments (MoM) numerical method.

4. Computation of Chain Parameters. Calculate the Chain Parameters Matrix from Capacitance Matrix for each uniform section.

5. Concatenation of Chain Parameters of Each Section. Calculate the Chain Parameters Matrix for the whole transmission line (original structure) by multiplication of individual section Chain Parameter Matrices. 
6. Computation of Impedance Parameters. Calculate the Impedance Parameters Matrix for the whole transmission line by transforming the Chain Parameters Matrix from previous step into Impedance Parameters.

The above steps are given as a flow chart in Figure 4.4.

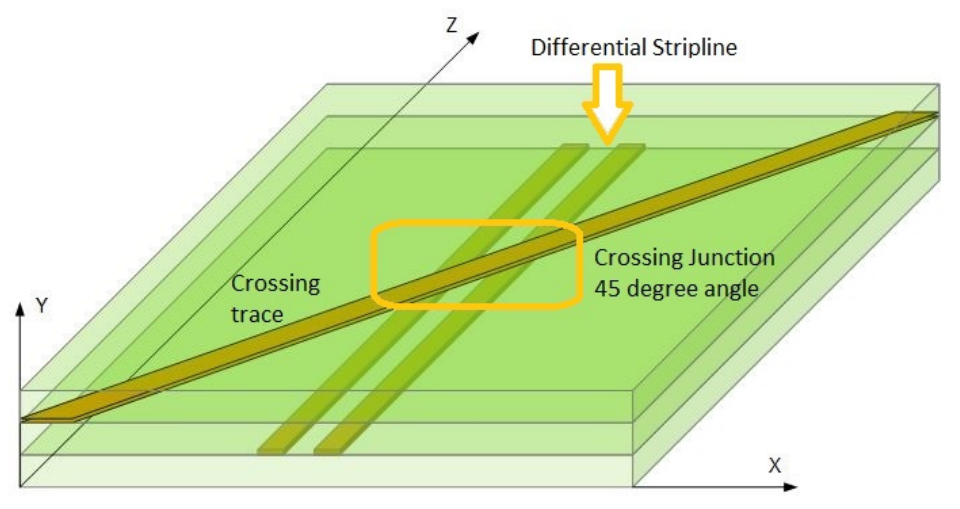

Figure 4.1: An Illustrative Differential Stripline with Crossing Trace at $45^{\circ}$ Angle 


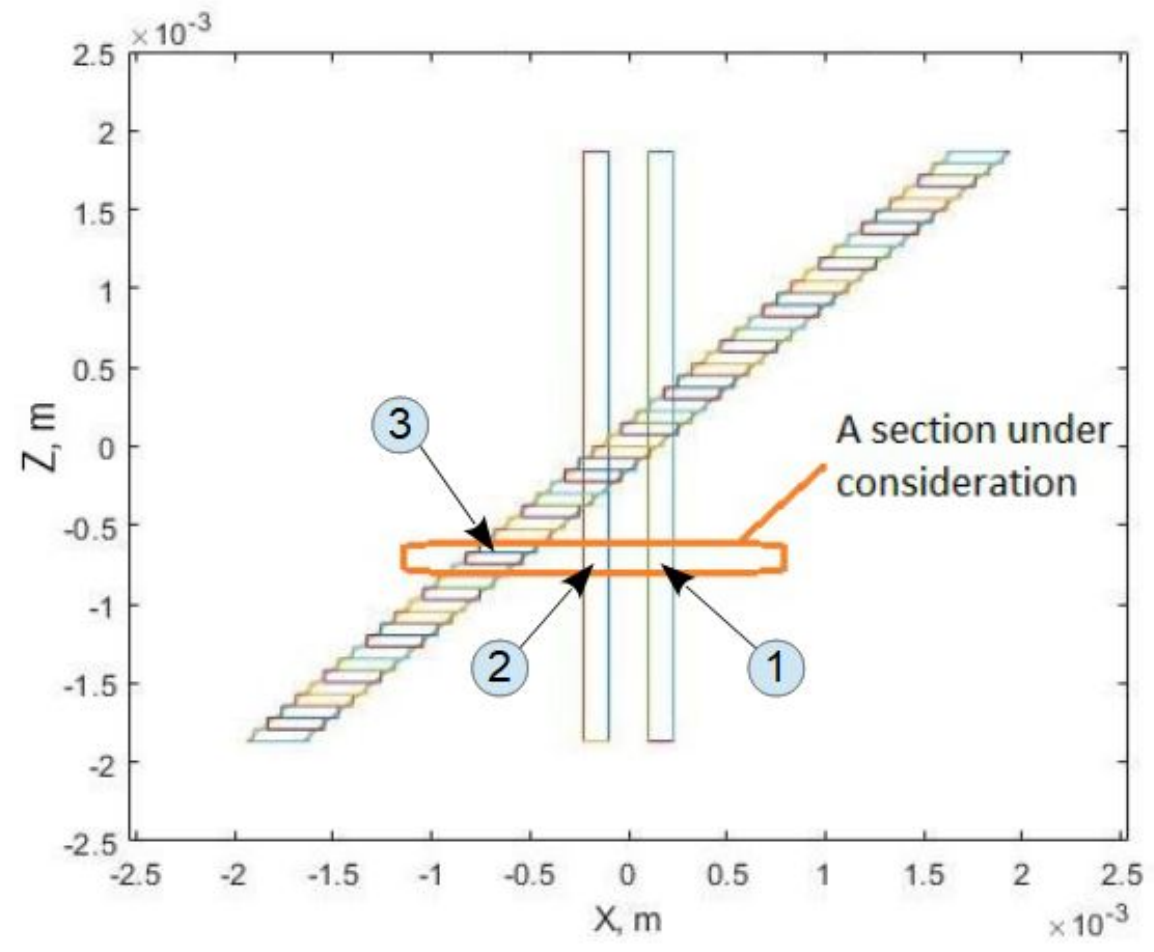

Figure 4.2: Slicing 3D Structure into $N_{s}$ Sections in XZ-plane and Approximating them with Uniform Sections. Circled (3) represents a sample section of the crossing trace and the corresponding nearest influenced region on the differential stripline is marked by entries (2) and (1). 


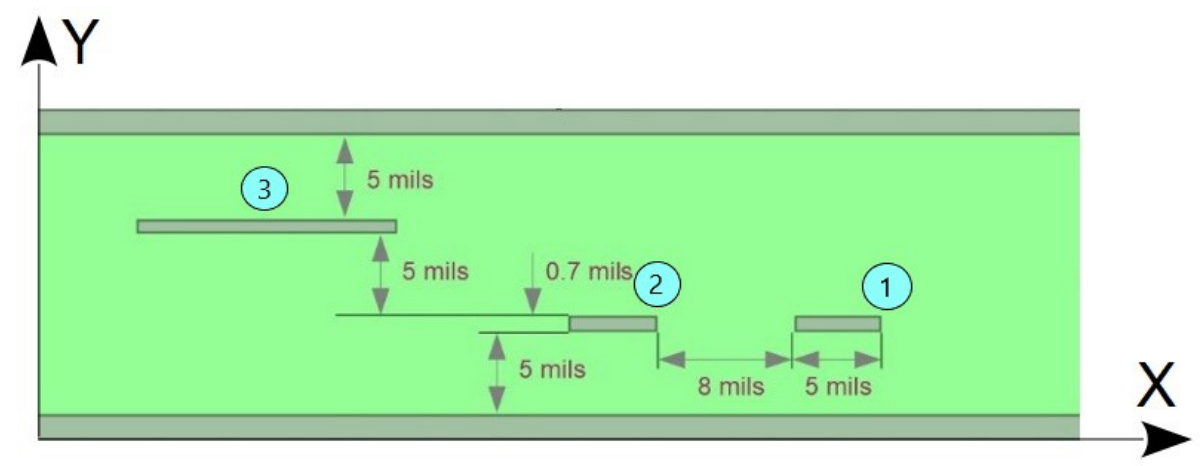

Figure 4.3: Cross-sectional View of One Sample Section in XY-plane: (1) and (2) Correspond to the Differential Stripline \& (3) Represents the Crossing Trace (see Figure 4.2 for additional information). 


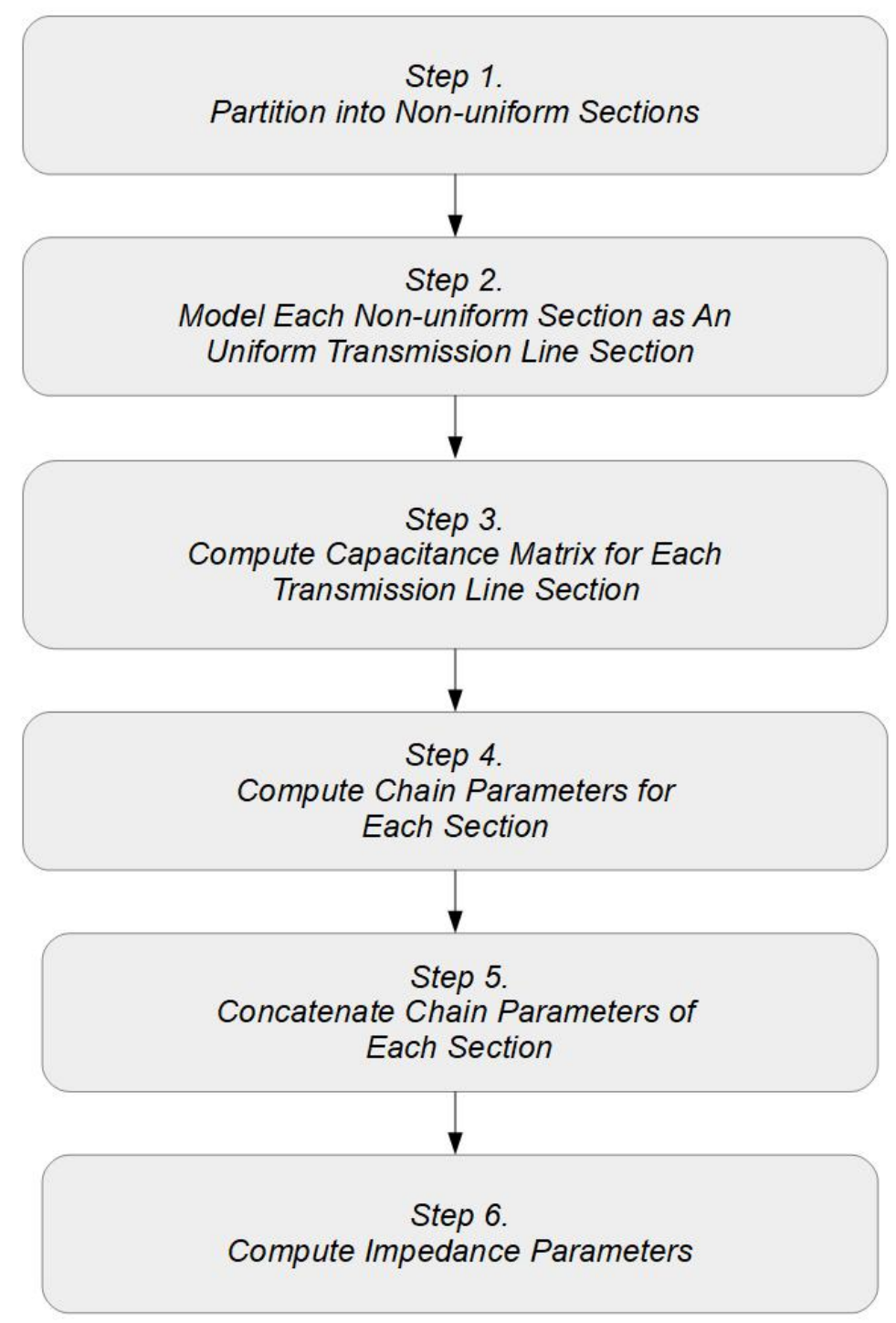

Figure 4.4: Proposed Solution for Oblique Crossing Problem 


\subsection{Computation of Capacitance Matrix}

Each section of Figure 4.3 consisting of three conductors and two reference planes can be considered as an uniform TEM structure and therefore can be solved using MoM solution to the integral equation for EM fields in that structure. The details of MoM solution for this structure [9] are described in this section.

\subsubsection{Solution of Integral Equation}

MoM is a method for solving the integral equation of the form:

$$
\int_{Z_{L}}^{Z_{U}} f\left(z^{\prime}\right) G\left(z, z^{\prime}\right) d z^{\prime}=g(z)
$$

where

$f(z)$ is unknown function

$g(z)$ is known function

$G\left(z, z^{\prime}\right)$ is Green's function, and

$Z_{L} \leq z \leq Z_{U}$

The expression (4.1) is an integral equation for the unknown function $f(z)$. In order to find an approximate solution for $f(z)$ we first expand $f(z)$ in terms of some $N$ selected known expansion functions $f_{n}(z)$ as

$$
f(z) \approx \sum_{n=1}^{N} a_{n} f_{n}(z)
$$

The coefficients $a_{n}, n=1,2, \ldots, N$ are unknown and MoM is used to find the coefficients in order to find the unknown function. If (4.2) is substituted into 
(4.1) the following expression is obtained

$$
\int_{Z_{L}}^{Z_{U}}\left(\sum_{n=1}^{N} a_{n} f_{n}\left(z^{\prime}\right)\right) G\left(z, z^{\prime}\right) d z^{\prime} \approx g(z)
$$

Next, the summation of the coefficients can be moved out of the integral as

$$
\sum_{n=1}^{N} a_{n}\left(\int_{Z_{L}}^{Z_{U}} f_{n}\left(z^{\prime}\right) G\left(z, z^{\prime}\right) d z^{\prime}\right) \approx g(z)
$$

which can be written as

$$
\sum_{n=1}^{N} a_{n} D_{n}(z)=g(z)
$$

where

$$
D_{n}(z)=\int_{Z_{L}}^{Z_{U}} f_{n}\left(z^{\prime}\right) G\left(z, z^{\prime}\right) d z^{\prime}
$$

The function $D_{n}(z)$ can be calculated for any value of $z$ since $f_{n}\left(z^{\prime}\right)$ and $G\left(z, z^{\prime}\right)$ are known. Next, we select a set of $N$ weighting functions $g_{m}(z), m=$ $1,2, \ldots, N$, multiply both sides of (4.5) by each of these weighting functions and take the integral with respect to $z$ as

$$
\sum_{n=1}^{N} a_{n}\left(\int_{Z_{L}}^{Z_{U}} D_{n}(z) g_{m}(z) d z\right)=\int_{Z_{L}}^{Z_{U}} g(z) g_{m}(z) d z
$$

The equation (4.7) can be written in a matrix form as

$$
[\mathbf{Z}][\mathbf{I}]=[\mathbf{V}]
$$

where

$$
\begin{aligned}
Z_{m n} & =\int_{Z_{L}}^{Z_{U}} D_{n}(z) g_{m}(z) d z \\
b_{m} & =\int_{Z_{L}}^{Z_{U}} g(z) g_{m}(z) d z
\end{aligned}
$$


The known impedance matrix is

$$
[\mathbf{Z}]=\left[\begin{array}{cccc}
Z_{11} & Z_{12} & \ldots & Z_{1 N} \\
Z_{21} & Z_{22} & \ldots & Z_{2 N} \\
\ldots & \ldots & \ldots & \ldots \\
Z_{N 1} & Z_{N 2} & \ldots & Z_{N N}
\end{array}\right]
$$

The known excitation column vector is

$$
[\mathbf{V}]=\left[\begin{array}{c}
b_{1} \\
b_{2} \\
\cdots \\
b_{N}
\end{array}\right]
$$

The column vector of unknown coefficients is

$$
[\mathbf{I}]=\left[\begin{array}{c}
a_{1} \\
a_{2} \\
\cdots \\
a_{N}
\end{array}\right]
$$

The goal of MoM is to find the $a_{n}$ coefficients given the $\mathbf{Z}$ and $\mathbf{V}$. Therefore, the equation (4.8) can be rewritten as:

$$
[\mathbf{I}]=[\mathbf{Z}]^{-1}[\mathbf{V}]
$$

\subsubsection{Segmentation of the Geometry}

As a first step in MoM based solution, the surfaces of each conductor and reference plane are segmented into a number of segments. The number of segments can be different for each conductor and reference plane. Larger 
number of segments give more accurate results at the expense of the computation time. For each segment, the $x, y$ coordinates for the start $x_{n}^{1}, y_{n}^{1}$ and end of segment $x_{n}^{2}, y_{n}^{2}$ are stored, where $n$ is the segment number. An example of segmented geometry for one section of the differential stripline in the presence of crossing trace is shown in Figure 4.5. It should be noted that in this example the conductors are assumed to be infinitely thin, segmenting the lines representing their surfaces. However, in case of a conductor with rectangular cross-section, it is possible to segment the contour of the rectangle to improve accuracy. Also, additional conductors can be modelled using this approach.

Let each conductor surface be a contour, denoted by $C_{n}: C_{1}, C_{2}, \ldots, C_{M}$, where $M$ is the number of conductors. In the example uniform structure (of Figure 4.5) there are 5 conductor contours:

$C_{1}$ - top reference conductor with $N_{1}$ segments

$C_{2}$ - bottom reference conductor with $N_{2}$ segments

$C_{3}$ - left conductor of stripline with $N_{3}$ segments

$C_{4}$ - right conductor of stripline with $N_{4}$ segments

$C_{5}$ - crossing conductor with $N_{5}$ segments

The total number of segments in this case:

$$
N=N_{1}+N_{2}+N_{3}+N_{4}+N_{5}
$$

Next, define

- $\Delta C_{n}$ to be the portion of the contour on any conductor that is occupied by $n-t h$ segment

- $\boldsymbol{\rho}$ to be the vector from the coordinate system origin to a point on the contour of any conductor. 
- $h_{n}$ to be the length of the $n-t h$ segment.

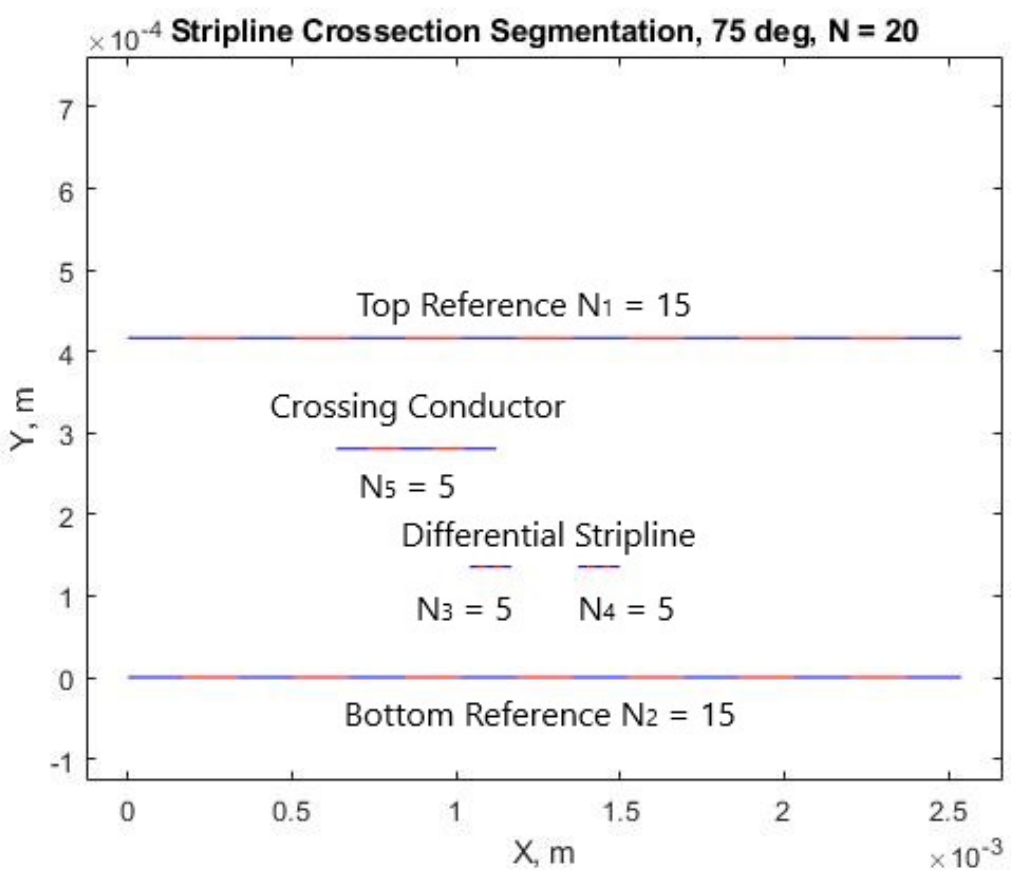

Figure 4.5: Segmentation of One Section of the Differential Stripline in the Presence of Crossing Trace

The reference diagram for the segmentation step with two segments is shown in Figure 4.6. 


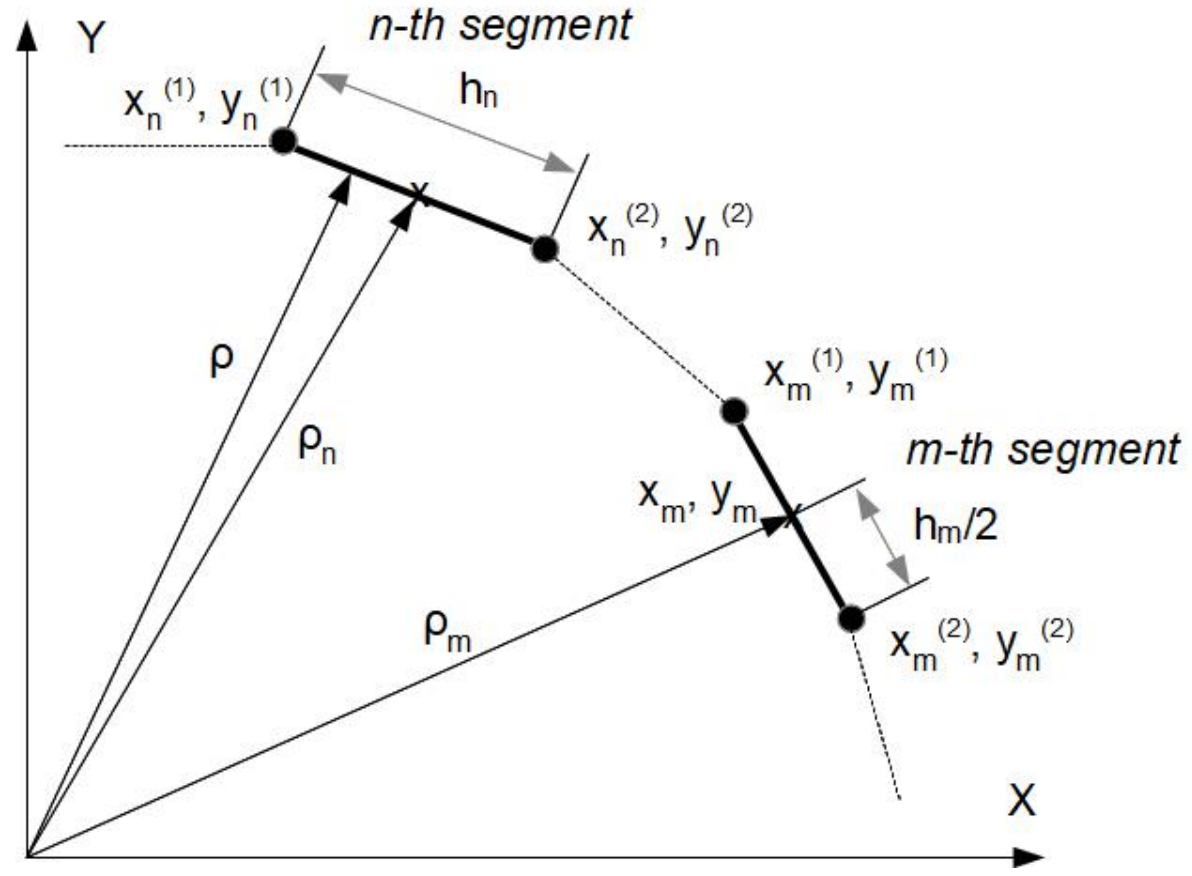

Figure 4.6: Reference Segmentation Diagram with Focus on Two Segments 


\subsubsection{Selection of the Expansion Functions}

In the proposed algorithm, "pulse" expansion functions are selected as:

$$
P_{n}(\boldsymbol{\rho})= \begin{cases}1 & \boldsymbol{\rho} \in \Delta C_{n} \\ 0 & \boldsymbol{\rho} \notin \Delta C_{n}\end{cases}
$$

The unknown function is surface charge density and can be expressed using (4.2) as

$$
\sigma(\boldsymbol{\rho})=\sum_{n=1}^{N} a_{n} P_{n}(\boldsymbol{\rho})
$$

\subsubsection{Selection of the Weighting Functions}

For this purpose, delta functions are selected as weighting functions:

$$
g_{m}(\boldsymbol{\rho})=\delta\left(\boldsymbol{\rho}-\boldsymbol{\rho}_{\boldsymbol{m}}\right)
$$

\subsubsection{Evaluation of the Excitation Matrix Terms}

The excitation matrix elements are expressed by (4.10). With the selected weighting functions, this expression evaluates to

$V_{m}=\int_{C_{e q}} V(\boldsymbol{\rho}) g_{m}(\boldsymbol{\rho}) d C=\int_{C_{e q}} V(\boldsymbol{\rho}) \delta\left(\boldsymbol{\rho}-\boldsymbol{\rho}_{\boldsymbol{m}}\right) d C=V\left(\boldsymbol{\rho}_{\boldsymbol{m}}\right)=\left\{\begin{array}{cc}V_{1} & \boldsymbol{\rho}_{\boldsymbol{m}} \in C_{1} \\ V_{2} & \boldsymbol{\rho}_{\boldsymbol{m}} \in C_{2} \\ \ldots & \ldots \\ V_{M} & \boldsymbol{\rho}_{\boldsymbol{m}} \in C_{M}\end{array}\right.$

where $M$ is the number of conductors. 


\subsubsection{Evaluation of the Impedance Matrix Terms}

The impedance matrix $\mathbf{Z}$ is evaluated using the expression (4.9) as [9]:

$Z_{m n}=-\frac{h_{n}}{4 \pi \varepsilon} \int_{0}^{1} \ln \left(\left\{x_{m}-x_{n}^{(1)}-\xi\left(x_{n}^{(2)}-x_{n}^{(1)}\right)\right\}^{2}+\left\{y_{m}-y_{n}^{(1)}-\xi\left(y_{n}^{(2)}-y_{n}^{(1)}\right)\right\}^{2}\right) d \xi$

where $x_{m}$ and $y_{m}$ are the coordinates of the middle of the segment $m$ :

$$
\begin{aligned}
& x_{m}=\left(x_{m}^{(1)}+x_{m}^{(2)}\right) / 2 \\
& y_{m}=\left(y_{m}^{(1)}+y_{m}^{(2)}\right) / 2
\end{aligned}
$$

The diagonal elements of the $\mathbf{Z}$ matrix are calculated as follows:

$$
Z_{n n}=-\frac{h_{n}}{2 \pi \varepsilon}\left(\ln \frac{h_{n}}{2}-1\right)
$$

Since all the segments in the structure of interest are horizontal (see Figure 4.5), the following relations can be simplified as

$$
\begin{gathered}
x_{n}^{(2)}-x_{n}^{(1)}=h_{n} \\
y_{n}^{(2)}-y_{n}^{(1)}=0 \\
y_{m}=y_{m}^{(1)}=y_{m}^{(2)}
\end{gathered}
$$

With these simplifications, the equation (4.19) can be written as

$$
Z_{m n}=-\frac{h_{n}}{4 \pi \varepsilon} \int_{0}^{1} \ln \left(\left\{x_{m}-x_{n}^{(1)}-\xi h_{n}\right\}^{2}+\left\{y_{m}^{(1)}-y_{n}^{(1)}\right\}^{2}\right) d \xi
$$




\subsubsection{Evaluation of the PUL Charge}

Once the $a_{n}$ coefficients are known from the equation (4.14), the charge per unit length for each conductor can be calculated with substitution from (4.16) as follows:

$q_{i}=\int_{C_{n}} \sigma(\boldsymbol{\rho}) d C=\int_{C_{n}}\left(\sum_{n=1}^{N_{i}} a_{n} P_{n}(\boldsymbol{\rho})\right) d C=\sum_{n=1}^{N_{i}} a_{n} h_{n}=a_{1} h_{1}+a_{2} h_{2}+\ldots+a_{N_{i}} h_{N_{i}}$

where $N_{i}$ is the number of segments on conductor $i$.

\subsubsection{Evaluation of the PUL Capacitance}

With the charge per unit length known using (4.27), elements of the capacitance matrix can be calculated using the equation (2.16), as follows:

$$
\begin{gathered}
q_{1}=C_{11} V_{1}+C_{12} V_{2}+\ldots+C_{1 N} V_{N} \\
q_{2}=C_{21} V_{1}+C_{22} V_{2}+\ldots+C_{2 N} V_{N} \\
q_{n}=C_{N 1} V_{1}+C_{N 2} V_{2}+\ldots+C_{N N} V_{N}
\end{gathered}
$$

where $C_{i j}$ are elements of the capacitance matrix $\mathbf{C}$. To calculate the capacitance matrix elements, we set the voltage on conductor $j$ to $V_{j}=1 V$ and on the other conductors and reference planes to $0 \mathrm{~V}$ to define the excitation matrix V. Subsequently, $\mathbf{Z}$ matrix is calculated and the unknown $a_{n}$ coefficients are found to calculate the charge per unit length $q_{i}$ as described in the previous sections. Next, the elements of the capacitance matrix can be calculated as follows:

$$
C_{i j}=\frac{q_{i}}{V_{j}}=q_{i} ; V_{j}=1 ; V_{k \neq j}=0
$$


where $i, j, k$ belong to symbol $1,2,3$.

$$
\mathbf{C}=\left[\begin{array}{lll}
C_{11} & C_{12} & C_{13} \\
C_{21} & C_{22} & C_{23} \\
C_{31} & C_{32} & C_{33}
\end{array}\right]
$$




\section{Chapter 5}

\section{Numerical Results}

This chapter presents the validating numerical results for the proposed method for efficient analysis of asymmetrical differential striplines in the presence of oblique crossing. The results are organized to provide the validation for each step of the algorithm. First, a differential stripline with practical dimensions in the presence of crossing trace at different angles is described and is divided into sections along the Z-axis. The diagrams of the sectionalized transmission lines with crossing traces are provided in Section 5.1. The capacitance matrix elements are calculated using the MoM and are plotted along the Z-axis while comparing with Ansys Maxwell 2D tool in Section 5.1.1. Section 5.1.2 provides the differential and common-mode impedances for lossless case along the Z-axis for crossing traces at different angles. Section 5.1.3 provides the comparison of $Z$ parameters for four different experiments using the proposed method and Ansys SI Wave tool.

Section 5.2 presents an investigation of impact of crossing junctions on signal integrity performance. Results are provided in terms of eye diagrams 
in the presence of crossings at $45^{\circ}, 90^{\circ}$ and no crossings.

\section{$5.1 \quad$ Example 1}

For the experiments in Section 5.1, differential stripline in the presence of crossing trace is considered with the cross-sectional setup in XY-plane as shown in Figure 5.1. The stripline dimensions were chosen to be the same as in [6] and [7] which represent the typical dimensions of traces on PCBs. The length of the crossing trace segment for this experiment is 200 mils, the width is 8 mils and the thickness is 0.7 mils. The selected number of uniform sections is $N_{s}=50$ to give reasonable accuracy and computational time. The crossing trace angles of $0^{\circ}, 15^{\circ}, 30^{\circ}, 45^{\circ}, 60^{\circ}, 75^{\circ}$ and $90^{\circ}$ were considered in the investigation. The plots of the sectionalized transmission line with crossing trace at these angles are shown in Figures 5.2 - 5.3.

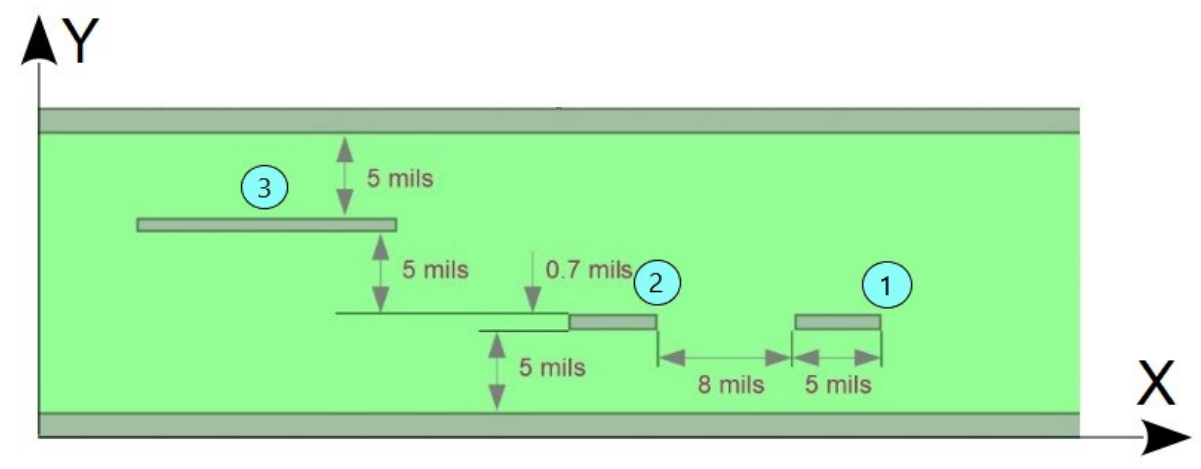

Figure 5.1: Cross-sectional View of One Sample Section in XY-plane: (1) and (2) Correspond to the Differential Stripline \& (3) Represents the Crossing Trace 


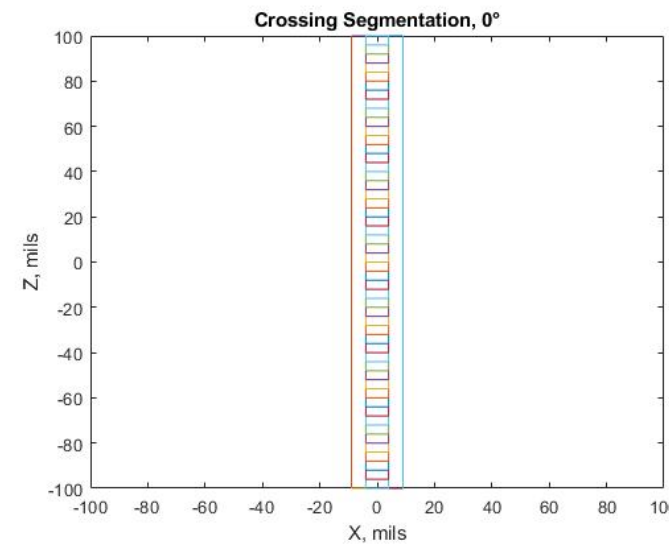

(a) $0^{\circ}$

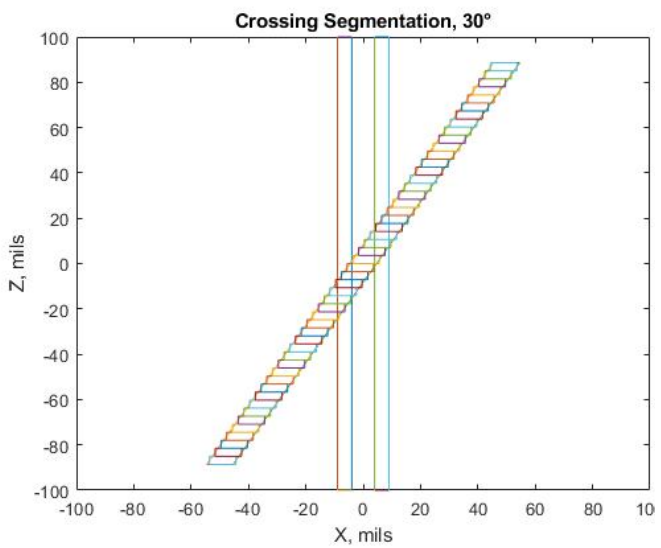

(c) $30^{\circ}$

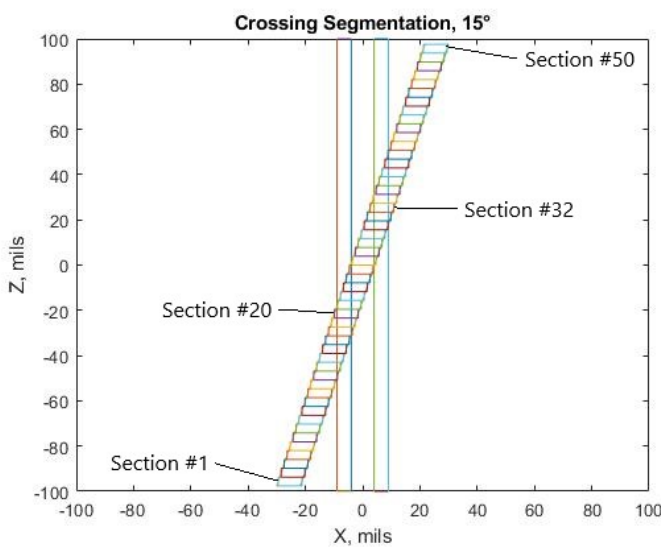

(b) $15^{\circ}$

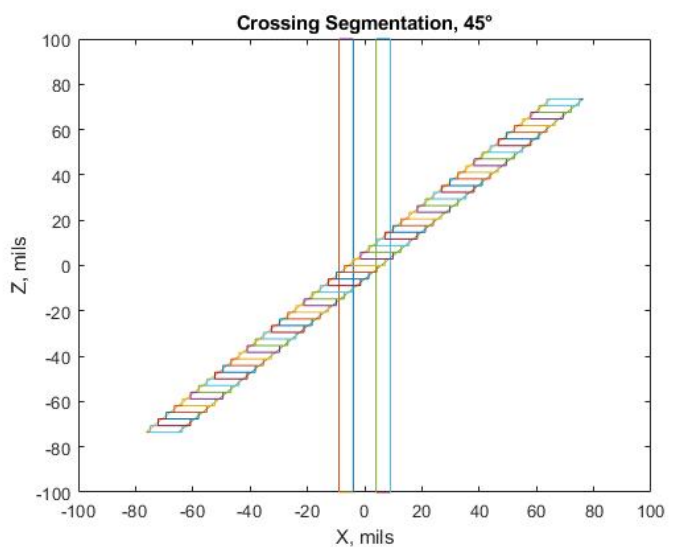

(d) $45^{\circ}$

Figure 5.2: Illustration of Differential Stripline with Crossing Trace at Different Angles $\left(0^{\circ}, 15^{\circ}, 30^{\circ}, 45^{\circ}\right)$ and its Segmentation into $N_{s}=50$ Sections 


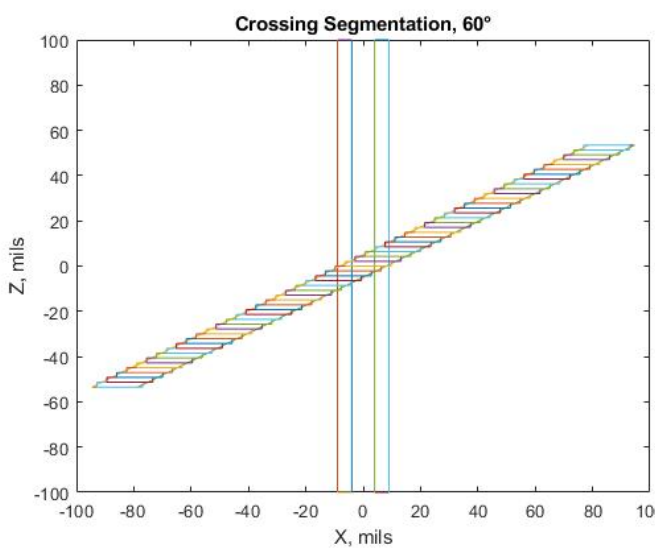

(a) $60^{\circ}$

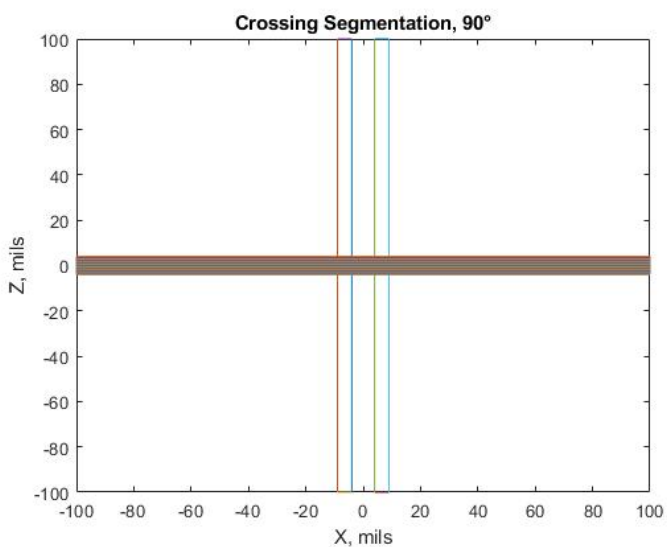

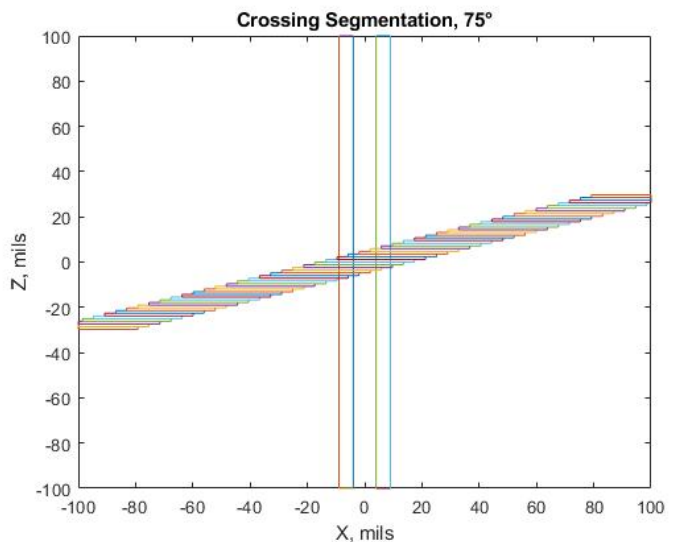

(b) $75^{\circ}$

(c) $90^{\circ}$

Figure 5.3: Illustration of Differential Stripline with Crossing Trace at Different Angles $\left(60^{\circ}, 75^{\circ}, 90^{\circ}\right)$ and its Segmentation into $N_{s}=50$ Sections 


\subsubsection{Capacitance Matrix Elements at Different An- gles}

The capacitance matrix along the Z-axis is computed using the proposed method. The plots of different capacitance matrix elements along the Z-axis for the differential ASL in the presence of crossing trace at different angles are shown in Figures $5.4-5.9$ (when using $N_{s}=50$ number of sections). Also, an error comparison of the capacitance matrix elements using the proposed method with the Ansys Maxwell 2D is made and the results are given in Tables $5.1-5.6$.

In these tables, results are organized with respect to varying sample angles as given in Column 1. For each angle, capacitances are measured with respect to different (sample) section position numbers (which are given in Column 2 ), and the corresponding capacitance values using the proposed method are reported in Column 3. For example, for crossing angle $15^{\circ}$ (please refer to Figure 5.2(b)), the sections are numbered from left-bottom (Section \#1) to top-right (Section \#50). Similar approach is taken while considering other angles and also other tables. In column 4 capacitance values obtained using Ansys Maxwell 2D are reported. In column 5 the percent error between the two methods is given. The results show reasonably good matching between the proposed approach and the Ansys Maxwell 2D. 


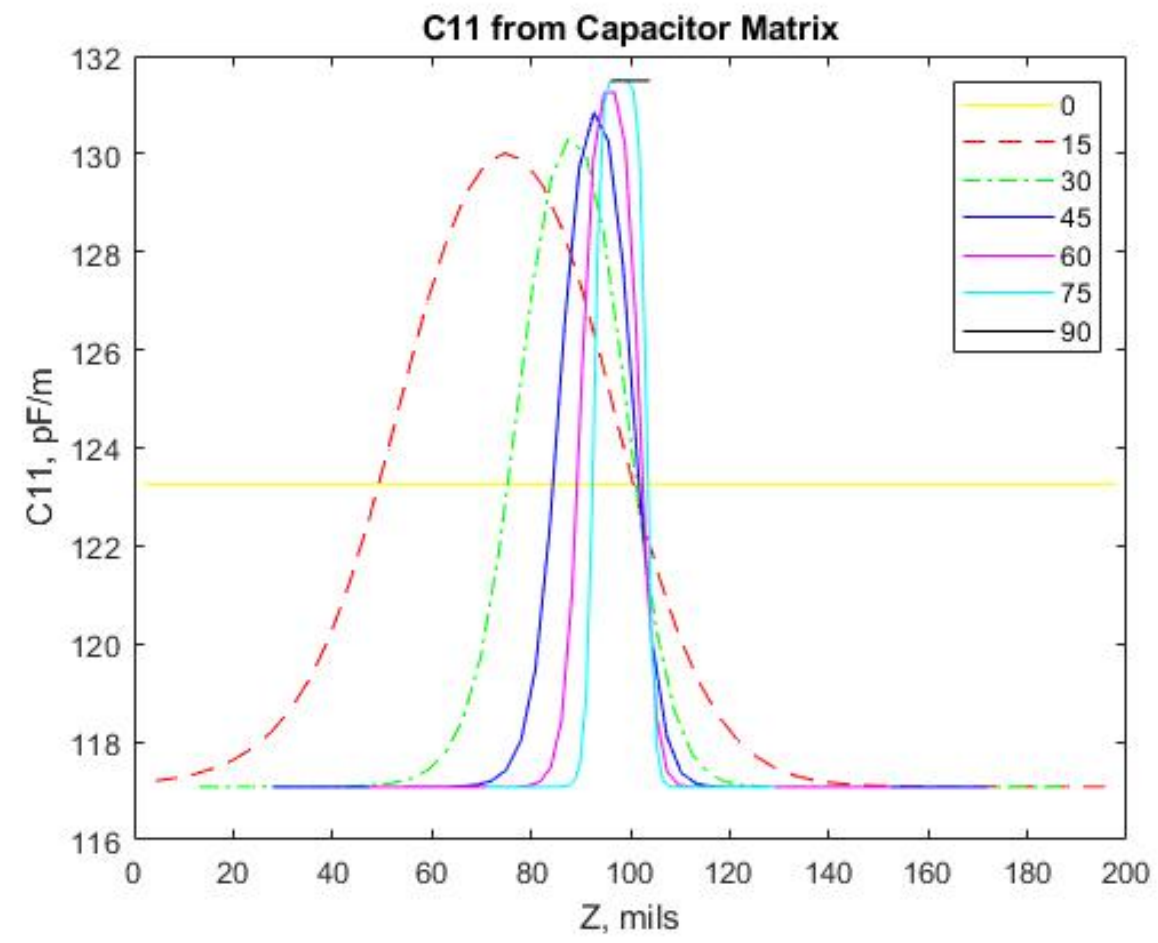

Figure 5.4: Variation (of $C_{11}$ Capacitance Matrix Element) for Different Crossing Trace Angles (with $N_{s}=50$ ) 
Table 5.1: Comparison of Capacitance Matrix Element $C_{11}$

\begin{tabular}{|c|c|c|c|c|}
\hline \multirow{2}{*}{ Trace Angle } & \multirow{2}{*}{$\begin{array}{c}\text { Section Position } \\
\text { Number }\end{array}$} & \multicolumn{2}{|c|}{$C_{11}(\mathrm{pF})$} & \multirow{2}{*}{$\%$ Error } \\
\hline & & Proposed & Maxwell 2D & \\
\hline $0^{\circ}$ & 1 & 123.27 & 124.72 & 1.17 \\
\hline $15^{\circ}$ & 1 & 117.20 & 118.54 & 1.13 \\
\hline $15^{\circ}$ & 20 & 129.86 & 131.46 & 1.21 \\
\hline $15^{\circ}$ & 32 & 117.69 & 119.05 & 1.14 \\
\hline $15^{\circ}$ & 50 & 117.08 & 118.42 & 1.13 \\
\hline $45^{\circ}$ & 1 & 117.08 & 118.57 & 1.26 \\
\hline $45^{\circ}$ & 20 & 122.43 & 124.10 & 1.34 \\
\hline $45^{\circ}$ & 32 & 117.09 & 118.58 & 1.26 \\
\hline $45^{\circ}$ & 50 & 117.08 & 118.57 & 1.26 \\
\hline $75^{\circ}$ & 1 & 117.08 & 118.76 & 1.41 \\
\hline $75^{\circ}$ & 20 & 128.19 & 130.26 & 1.59 \\
\hline $75^{\circ}$ & 32 & 117.10 & 118.78 & 1.42 \\
\hline $75^{\circ}$ & 50 & 117.08 & 118.76 & 1.41 \\
\hline $90^{\circ}$ & 1 & 131.49 & 133.56 & 1.55 \\
\hline
\end{tabular}




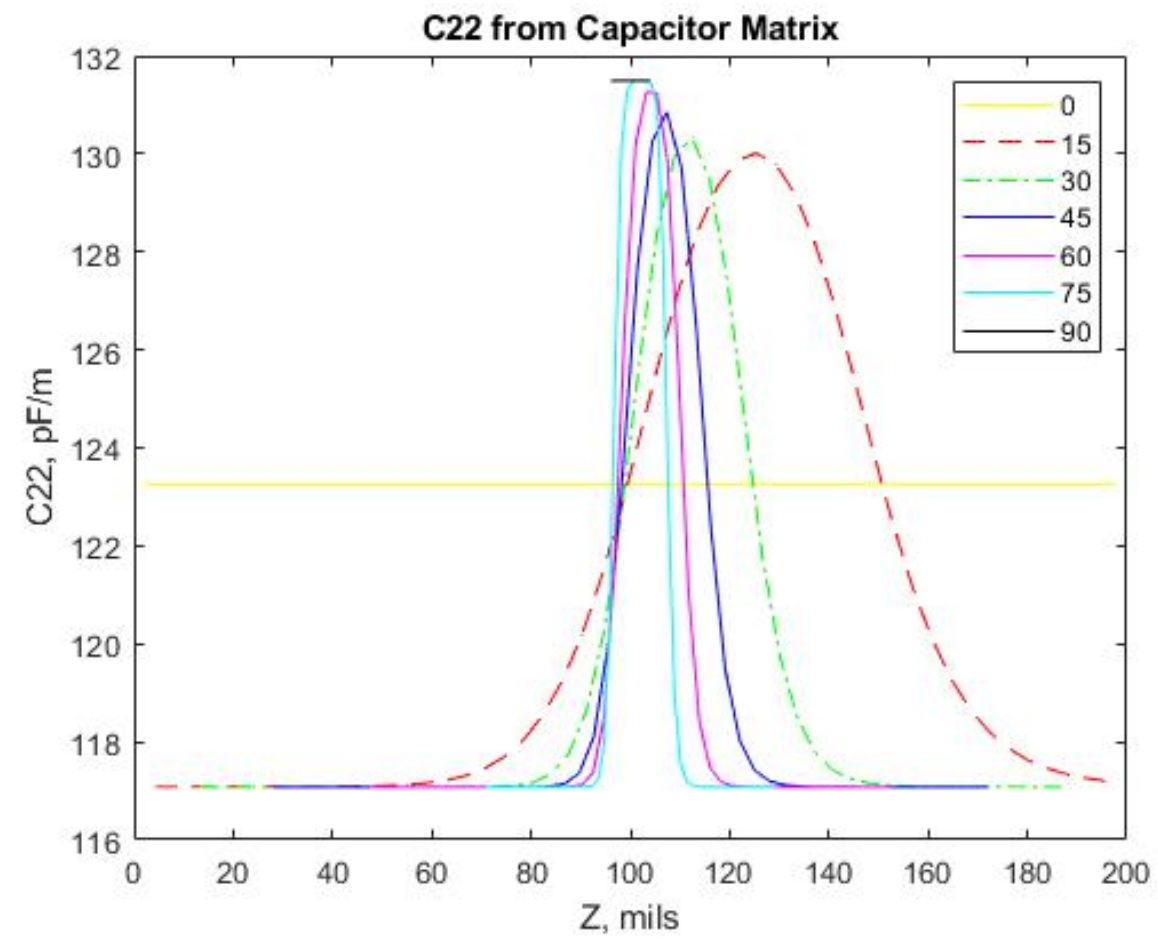

Figure 5.5: Variation (of $C_{22}$ Capacitance Matrix Element) for Different Crossing Trace Angles (with $N_{s}=50$ ) 
Table 5.2: Comparison of Capacitance Matrix Element $C_{22}$

\begin{tabular}{|c|c|c|c|c|}
\hline \multirow{2}{*}{ Trace Angle } & \multirow{2}{*}{$\begin{array}{c}\text { Section Position } \\
\text { Number }\end{array}$} & \multicolumn{2}{|c|}{$C_{22}(\mathrm{pF})$} & \multirow{2}{*}{$\%$ Error } \\
\hline & & Proposed & Maxwell 2D & \\
\hline $0^{\circ}$ & 1 & 123.27 & 124.72 & 1.17 \\
\hline $15^{\circ}$ & 1 & 117.08 & 118.42 & 1.13 \\
\hline $15^{\circ}$ & 20 & 118.05 & 119.41 & 1.14 \\
\hline $15^{\circ}$ & 32 & 130.01 & 131.61 & 1.21 \\
\hline $15^{\circ}$ & 50 & 117.20 & 118.54 & 1.13 \\
\hline $45^{\circ}$ & 1 & 117.08 & 118.57 & 1.26 \\
\hline $45^{\circ}$ & 20 & 117.10 & 118.58 & 1.25 \\
\hline $45^{\circ}$ & 32 & 119.47 & 121.04 & 1.30 \\
\hline $45^{\circ}$ & 50 & 117.08 & 118.57 & 1.26 \\
\hline $75^{\circ}$ & 1 & 117.08 & 118.71 & 1.37 \\
\hline $75^{\circ}$ & 20 & 117.21 & 118.84 & 1.38 \\
\hline $75^{\circ}$ & 32 & 122.71 & 124.62 & 1.53 \\
\hline $75^{\circ}$ & 50 & 117.08 & 118.71 & 1.37 \\
\hline $90^{\circ}$ & 1 & 131.49 & 133.48 & 1.49 \\
\hline
\end{tabular}




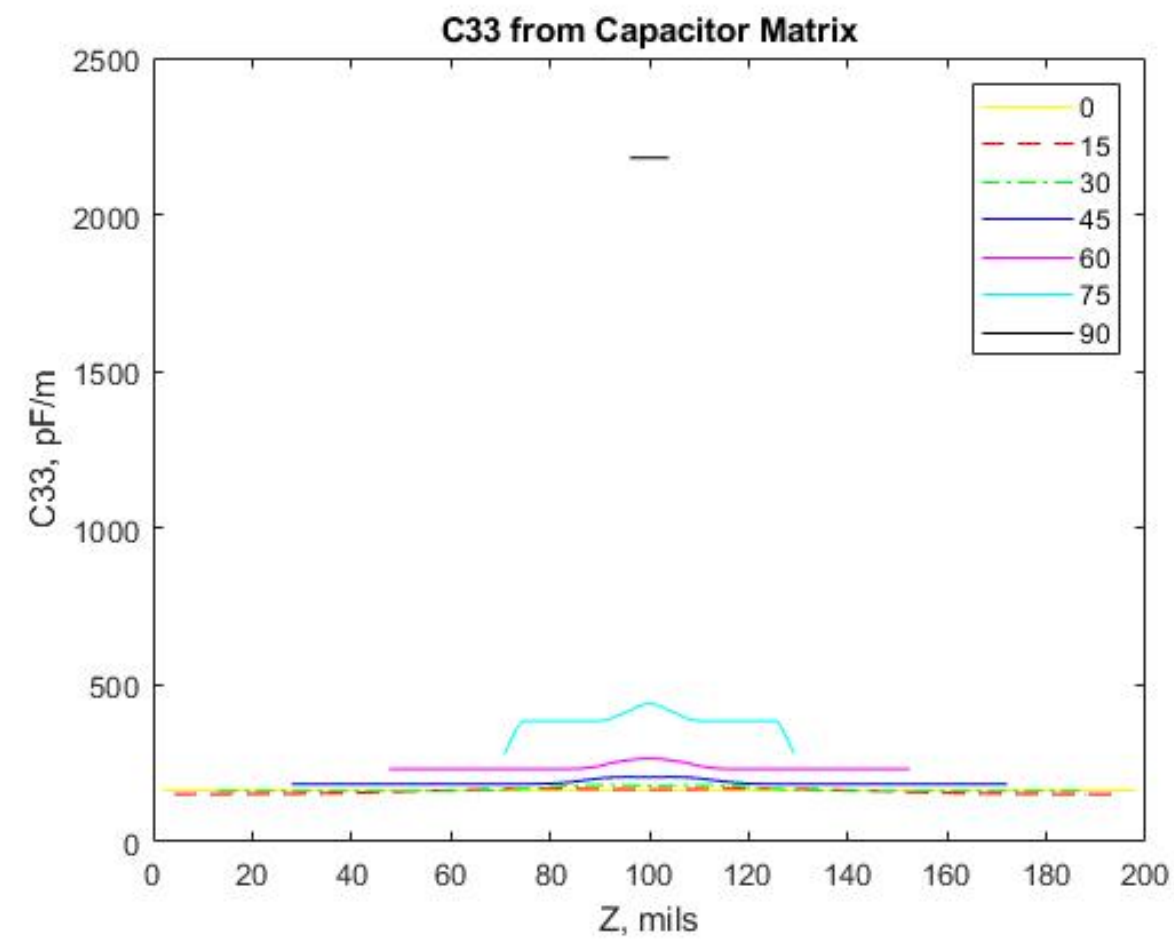

Figure 5.6: Variation (of $C_{33}$ Capacitance Matrix Element) for Different Crossing Trace Angles (with $N_{s}=50$ ) 
Table 5.3: Comparison of Capacitance Matrix Element $C_{33}$

\begin{tabular}{|c|c|c|c|c|}
\hline \multirow{2}{*}{ Trace Angle } & \multirow{2}{*}{$\begin{array}{c}\text { Section Position } \\
\text { Number }\end{array}$} & \multicolumn{2}{|c|}{$C_{33}(\mathrm{pF})$} & \multirow{2}{*}{$\%$ Error } \\
\hline & & Proposed & Maxwell 2D & \\
\hline $0^{\circ}$ & 1 & 163.56 & 164.98 & 0.86 \\
\hline $15^{\circ}$ & 1 & 152.17 & 153.30 & 0.74 \\
\hline $15^{\circ}$ & 20 & 169.80 & 171.26 & 0.85 \\
\hline $15^{\circ}$ & 32 & 169.62 & 171.08 & 0.85 \\
\hline $15^{\circ}$ & 50 & 152.17 & 153.30 & 0.74 \\
\hline $45^{\circ}$ & 1 & 183.65 & 185.29 & 0.89 \\
\hline $45^{\circ}$ & 20 & 190.11 & 191.79 & 0.87 \\
\hline $45^{\circ}$ & 32 & 186.43 & 188.05 & 0.86 \\
\hline $45^{\circ}$ & 50 & 183.65 & 185.33 & 0.91 \\
\hline $75^{\circ}$ & 1 & 281.28 & 283.78 & 0.88 \\
\hline $75^{\circ}$ & 20 & 401.27 & 404.77 & 0.87 \\
\hline $75^{\circ}$ & 32 & 392.24 & 395.56 & 0.84 \\
\hline $75^{\circ}$ & 50 & 281.28 & 283.86 & 0.91 \\
\hline $90^{\circ}$ & 1 & 2180.91 & 2195.60 & 0.67 \\
\hline
\end{tabular}




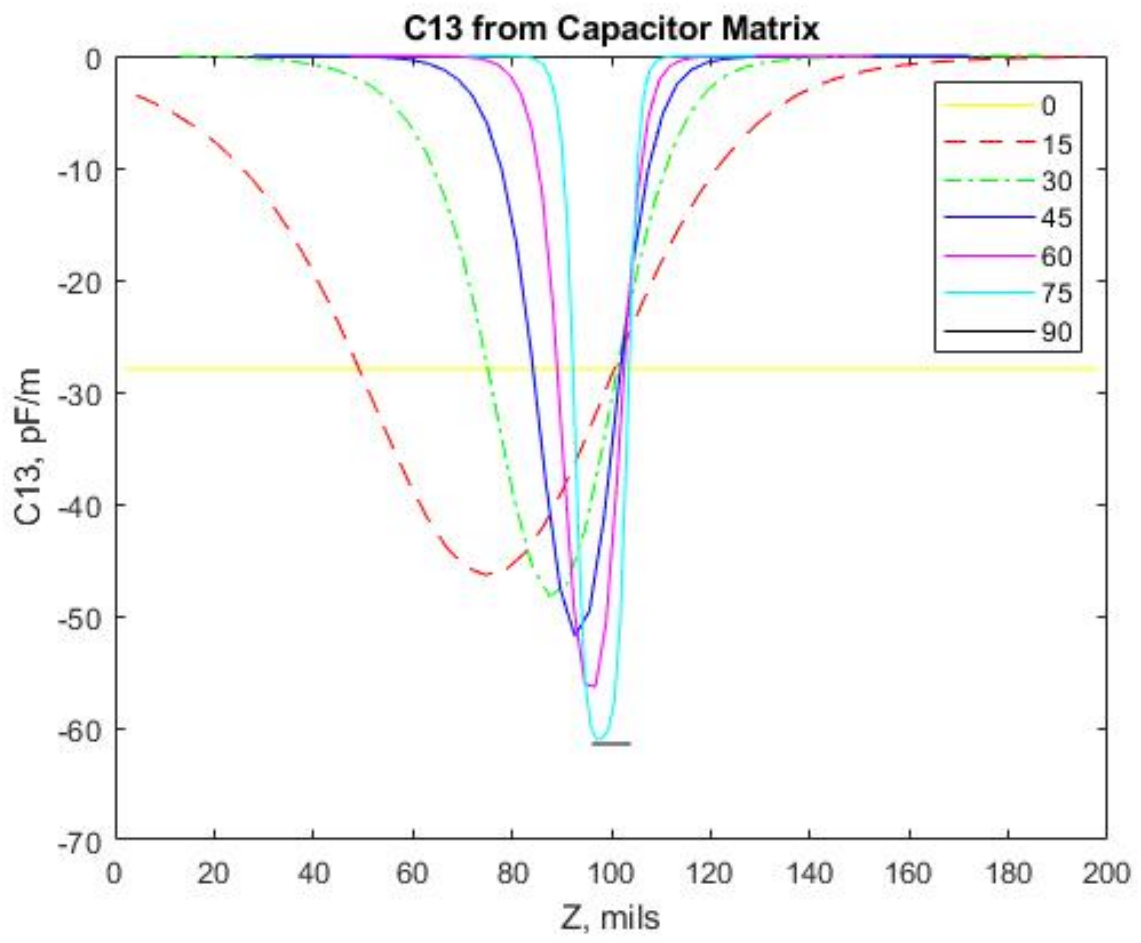

Figure 5.7: Variation (of $C_{13}$ Capacitance Matrix Element) for Different Crossing Trace Angles (with $N_{s}=50$ ) 
Table 5.4: Comparison of Capacitance Matrix Element $C_{13}$

\begin{tabular}{|c|c|c|c|c|}
\hline \multirow{2}{*}{ Trace Angle } & \multirow{2}{*}{$\begin{array}{c}\text { Section Position } \\
\text { Number }\end{array}$} & \multicolumn{2}{|c|}{$C_{13}(\mathrm{pF})$} & \multirow{2}{*}{$\%$ Error } \\
\hline & & Proposed & Maxwell 2D & \\
\hline $0^{\circ}$ & 1 & $-2.79 \mathrm{E}+01$ & $-2.84 \mathrm{E}+01$ & 1.60 \\
\hline $15^{\circ}$ & 1 & $-3.54 \mathrm{E}+00$ & $-3.63 \mathrm{E}+00$ & 2.37 \\
\hline $15^{\circ}$ & 20 & $-4.59 \mathrm{E}+01$ & $-4.66 \mathrm{E}+01$ & 1.41 \\
\hline $15^{\circ}$ & 32 & $-7.82 \mathrm{E}+00$ & $-7.96 \mathrm{E}+00$ & 1.83 \\
\hline $15^{\circ}$ & 50 & $-1.03 \mathrm{E}-01$ & $-1.05 \mathrm{E}-01$ & 1.82 \\
\hline $45^{\circ}$ & $\begin{array}{c}--- \\
1\end{array}$ & $-1.07 \mathrm{E}-03$ & $\begin{array}{l}-1.11 \mathrm{E}-03 \\
\end{array}$ & 4.19 \\
\hline $45^{\circ}$ & 20 & $-2.64 \mathrm{E}+01$ & $-2.70 \mathrm{E}+01$ & 2.09 \\
\hline $45^{\circ}$ & 32 & $-6.14 \mathrm{E}-01$ & $-6.27 \mathrm{E}-01$ & 2.00 \\
\hline $45^{\circ}$ & 50 & $-2.90 \mathrm{E}-05$ & $-3.03 \mathrm{E}-05$ & 4.21 \\
\hline $75^{\circ}$ & 1 & $-5.49 \mathrm{E}-05$ & $-5.73 \mathrm{E}-05$ & 4.12 \\
\hline $75^{\circ}$ & 20 & $-4.40 \mathrm{E}+01$ & $-4.50 \mathrm{E}+01$ & 2.25 \\
\hline $75^{\circ}$ & 32 & $-1.28 \mathrm{E}+00$ & $-1.32 \mathrm{E}+00$ & 3.24 \\
\hline $75^{\circ}$ & 50 & $-1.50 \mathrm{E}-06$ & $-1.55 \mathrm{E}-06$ & 3.35 \\
\hline $90^{\circ}$ & 1 & $-6.14 \mathrm{E}+01$ & $-6.24 \mathrm{E}+01$ & 1.54 \\
\hline
\end{tabular}




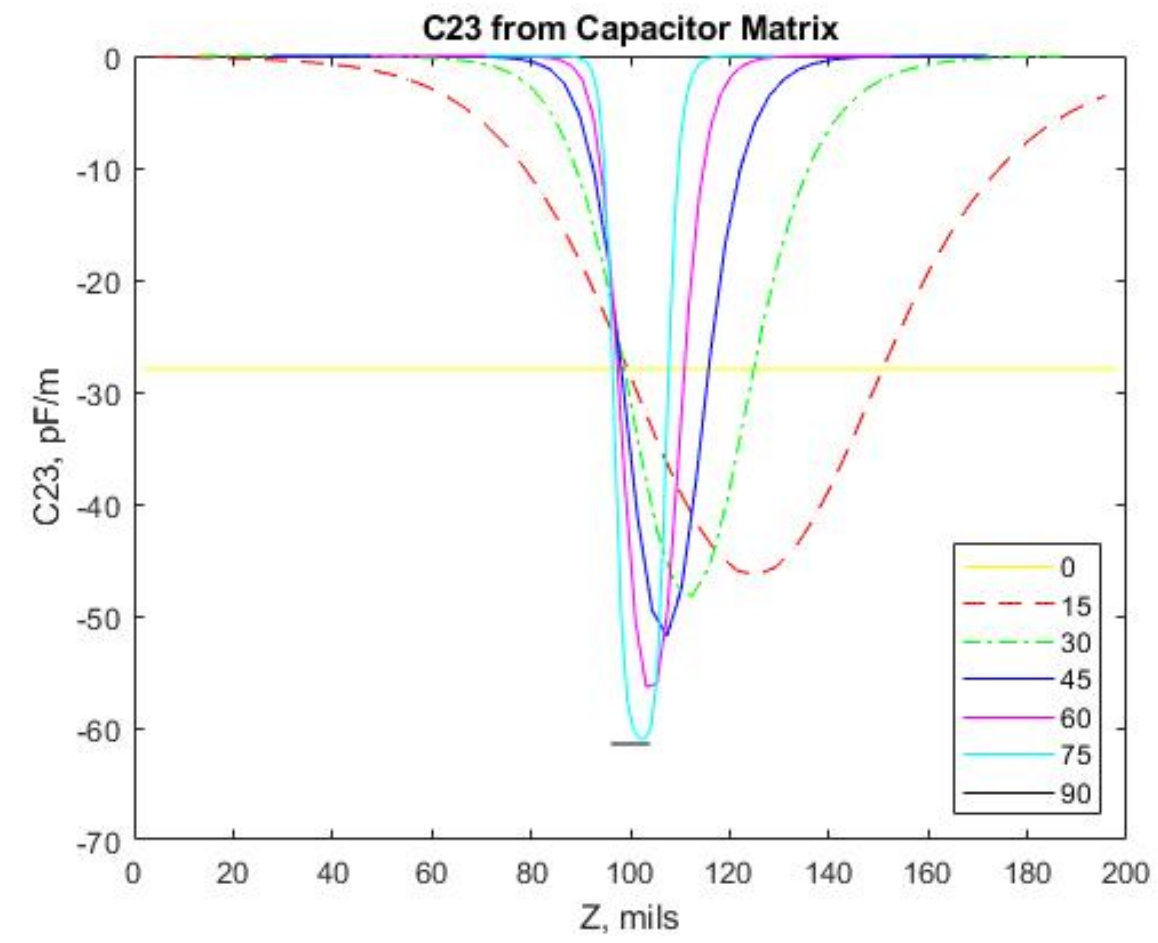

Figure 5.8: Variation (of $C_{23}$ Capacitance Matrix Element) for Different Crossing Trace Angles (with $N_{s}=50$ ) 
Table 5.5: Comparison of Capacitance Matrix Element $C_{23}$

\begin{tabular}{|c|c|c|c|c|}
\hline \multirow{2}{*}{ Trace Angle } & \multirow{2}{*}{$\begin{array}{c}\text { Section Position } \\
\text { Number }\end{array}$} & \multicolumn{2}{|c|}{$C_{23}(\mathrm{pF})$} & \multirow{2}{*}{$\%$ Error } \\
\hline & & Proposed & Maxwell 2D & \\
\hline $0^{\circ}$ & 1 & $-2.79 \mathrm{E}+01$ & $-2.84 \mathrm{E}+01$ & 1.58 \\
\hline $15^{\circ}$ & 1 & $-1.03 \mathrm{E}-01$ & $-1.06 \mathrm{E}-01$ & 3.46 \\
\hline $15^{\circ}$ & 20 & $-9.92 \mathrm{E}+00$ & $-1.01 \mathrm{E}+01$ & 1.80 \\
\hline $15^{\circ}$ & 32 & $-4.64 \mathrm{E}+01$ & $-4.70 \mathrm{E}+01$ & 1.42 \\
\hline $15^{\circ}$ & 50 & $-3.54 \mathrm{E}+00$ & $-3.63 \mathrm{E}+00$ & 2.37 \\
\hline $45^{\circ}$ & $\begin{array}{c}--- \\
1\end{array}$ & $-2.90 \mathrm{E}-05$ & $\begin{array}{c}-3.03 \mathrm{E}-05 \\
-\end{array}$ & 4.20 \\
\hline $45^{\circ}$ & 20 & $-1.21 \mathrm{E}+00$ & $-1.23 \mathrm{E}+00$ & 2.05 \\
\hline $45^{\circ}$ & 32 & $-1.68 \mathrm{E}+01$ & $-1.72 \mathrm{E}+01$ & 2.29 \\
\hline $45^{\circ}$ & 50 & $-1.07 \mathrm{E}-03$ & $-1.11 \mathrm{E}-03$ & 4.19 \\
\hline $75^{\circ}$ & 1 & $-1.50 \mathrm{E}-06$ & $-1.55 \mathrm{E}-06$ & 3.19 \\
\hline $75^{\circ}$ & 20 & $-3.48 \mathrm{E}+00$ & $-3.60 \mathrm{E}+00$ & 3.27 \\
\hline $75^{\circ}$ & 32 & $-2.77 \mathrm{E}+01$ & $-2.85 \mathrm{E}+01$ & 2.85 \\
\hline $75^{\circ}$ & 50 & $-5.49 \mathrm{E}-05$ & $-5.73 \mathrm{E}-05$ & 4.11 \\
\hline $90^{\circ}$ & 1 & $-6.14 \mathrm{E}+01$ & $-6.23 \mathrm{E}+01$ & 1.48 \\
\hline
\end{tabular}




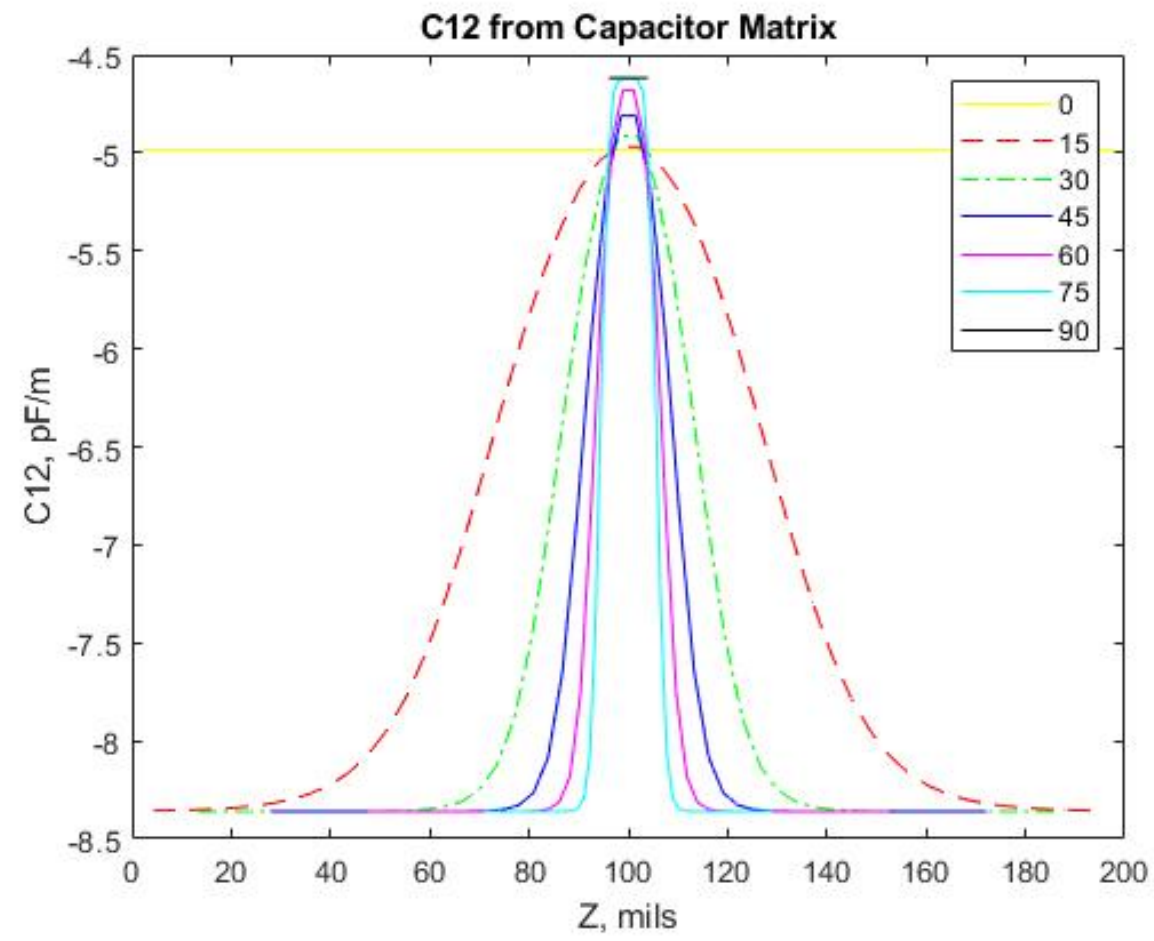

Figure 5.9: Variation (of $C_{12}$ Capacitance Matrix Element) for Different Crossing Trace Angles (with $N_{s}=50$ ) 
Table 5.6: Comparison of Capacitance Matrix Element $C_{12}$

\begin{tabular}{|c|c|c|c|c|}
\hline \multirow{2}{*}{ Trace Angle } & \multirow{2}{*}{$\begin{array}{c}\text { Section Position } \\
\text { Number }\end{array}$} & \multicolumn{2}{|c|}{$C_{12}(\mathrm{pF})$} & \multirow{2}{*}{ \% Error } \\
\hline & & Proposed & Maxwell 2D & \\
\hline $0^{\circ}$ & 1 & -4.986 & -5.150 & 3.18 \\
\hline $15^{\circ}$ & 1 & -8.357 & -8.599 & 2.81 \\
\hline $15^{\circ}$ & 20 & -5.948 & -6.133 & 3.01 \\
\hline $15^{\circ}$ & 32 & -6.285 & -6.478 & 2.97 \\
\hline $15^{\circ}$ & 50 & -8.357 & -8.599 & 2.81 \\
\hline $45^{\circ}$ & 1 & -8.360 & -8.633 & 3.16 \\
\hline $45^{\circ}$ & 20 & -8.085 & -8.348 & 3.15 \\
\hline $45^{\circ}$ & 32 & -8.266 & -8.536 & 3.17 \\
\hline $45^{\circ}$ & 50 & -8.360 & -8.633 & 3.16 \\
\hline $75^{\circ}$ & 1 & -8.360 & -8.658 & 3.44 \\
\hline $75^{\circ}$ & 20 & -7.332 & -7.586 & 3.34 \\
\hline $75^{\circ}$ & 32 & -8.064 & -8.348 & 3.40 \\
\hline $75^{\circ}$ & 50 & -8.360 & -8.658 & 3.44 \\
\hline $90^{\circ}$ & 1 & -4.618 & -4.828 & 4.34 \\
\hline
\end{tabular}




\subsubsection{Differential and Common-Mode Impedances for Lossless Case at Different Angles}

Using the capacitance matrices computed in the previous section, differential and common-mode impedances are computed. The plots for the differential and common-mode impedances along the Z-axis for the transmission line with the crossing trace at different angles are shown in Figure 5.10 and 5.11, respectively. Also, an error comparison for the differential and commonmode impedances between the proposed method and the Ansys Maxwell 2D are shown in Tables 5.7 and 5.8, respectively. Results in these tables are organized in a similar way as that in Table 5.1 (as described in Section 5.1.1). As seen from the tables, a reasonably good matching between both methods is observed. 


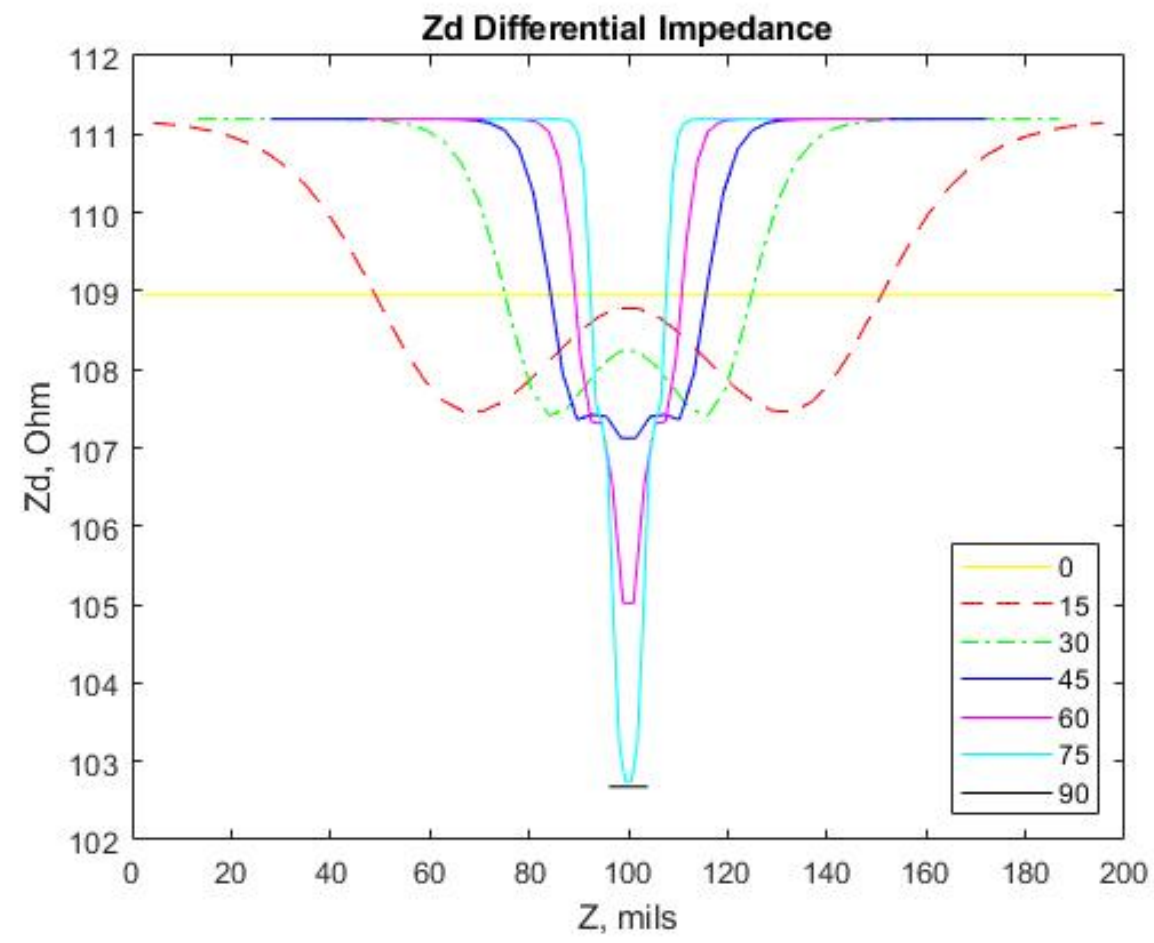

Figure 5.10: Comparative Study of Variation of Differential Impedance $Z_{d}$ for Different Crossing Trace Angles (with $N_{s}=50$ ) 
Table 5.7: Comparison of Differential Impedance $Z_{d}$

\begin{tabular}{|c|c|c|c|c|}
\hline \multirow{2}{*}{ Trace Angle } & \multirow{2}{*}{$\begin{array}{c}\text { Section Position } \\
\text { Number }\end{array}$} & \multicolumn{2}{|c|}{$Z_{d}(\mathrm{Ohm})$} & \multirow{2}{*}{$\%$ Error } \\
\hline & & Proposed & Maxwell 2D & \\
\hline $0^{\circ}$ & 1 & 109.04 & 107.68 & 1.26 \\
\hline $15^{\circ}$ & 1 & 111.43 & 110.04 & 1.26 \\
\hline $15^{\circ}$ & 20 & 107.88 & 106.52 & 1.28 \\
\hline $15^{\circ}$ & 32 & 107.71 & 106.35 & 1.28 \\
\hline $15^{\circ}$ & 50 & 111.43 & 110.04 & 1.26 \\
\hline $45^{\circ}$ & 1 & 111.48 & 109.94 & 1.40 \\
\hline $45^{\circ}$ & 20 & 109.43 & 107.88 & 1.44 \\
\hline $45^{\circ}$ & 32 & 110.52 & 108.97 & 1.42 \\
\hline $45^{\circ}$ & 50 & 111.48 & 109.94 & 1.40 \\
\hline $75^{\circ}$ & 1 & 111.48 & 109.77 & 1.56 \\
\hline $75^{\circ}$ & 20 & 107.75 & 106.04 & 1.61 \\
\hline $75^{\circ}$ & 32 & 109.33 & 107.59 & 1.62 \\
\hline $75^{\circ}$ & 50 & 111.48 & 109.77 & 1.56 \\
\hline $90^{\circ}$ & 1 & 102.74 & 101.08 & 1.64 \\
\hline
\end{tabular}




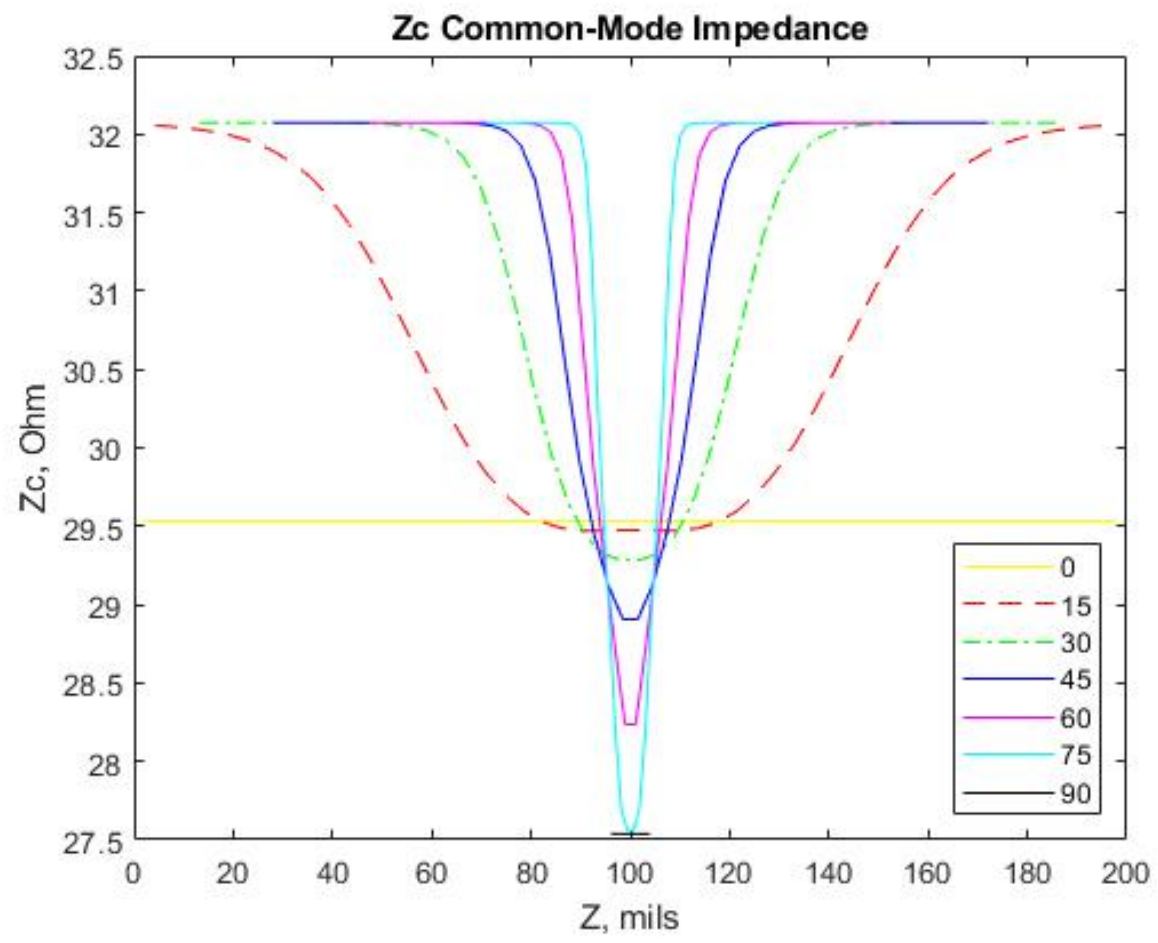

Figure 5.11: Comparative Study of Variation of Common-Mode Impedance $Z_{c}$ for Different Crossing Trace Angles (with $N_{s}=50$ ) 
Table 5.8: Comparison of Common-Mode Impedance $Z_{c}$

\begin{tabular}{|c|c|c|c|c|}
\hline \multirow{2}{*}{ Trace Angle } & \multirow{2}{*}{$\begin{array}{c}\text { Section Position } \\
\text { Number }\end{array}$} & \multicolumn{2}{|c|}{$Z_{c}(\mathrm{Ohm})$} & \multirow{2}{*}{$\%$ Error } \\
\hline & & Proposed & Maxwell 2D & \\
\hline $0^{\circ}$ & 1 & 29.56 & 29.24 & 1.09 \\
\hline $15^{\circ}$ & 1 & 32.14 & 31.82 & 1.01 \\
\hline $15^{\circ}$ & 20 & 29.63 & 29.30 & 1.10 \\
\hline $15^{\circ}$ & 32 & 29.74 & 29.41 & 1.09 \\
\hline $15^{\circ}$ & 50 & 32.14 & 31.82 & 1.01 \\
\hline $45^{\circ}$ & 1 & 32.16 & 31.80 & 1.12 \\
\hline $45^{\circ}$ & 20 & 31.30 & 30.94 & 1.17 \\
\hline $45^{\circ}$ & 32 & 31.78 & 31.42 & 1.15 \\
\hline $45^{\circ}$ & 50 & 32.16 & 31.80 & 1.12 \\
\hline $75^{\circ}$ & 1 & 32.16 & 31.76 & 1.25 \\
\hline $75^{\circ}$ & 20 & 30.30 & 29.89 & 1.39 \\
\hline $75^{\circ}$ & 32 & 31.26 & 30.84 & 1.35 \\
\hline $75^{\circ}$ & 50 & 32.16 & 31.76 & 1.25 \\
\hline $90^{\circ}$ & 1 & 27.56 & 27.17 & 1.43 \\
\hline
\end{tabular}




\subsubsection{Computation of $Z$ Parameters}

In this section, the ASL cross-section of Figure 5.1 is used in four different experimental setups. In these experiments, using the capacitance matrices computed in section 5.1.1, $Z$ parameters are computed as described in Section 2.3 and compared with the Ansys SI Wave tool.

\section{Experiment 1: ASL with One Crossing Junction at $45^{\circ}$.}

In this experiment, the proposed model for ASL is investigated in the presence of one crossing junction at $45^{\circ}$ segmented with $N_{s}=50$. The setup for this experiment is shown in Figure 5.12. $Z$ parameters for the differential stripline in the presence of one $45^{\circ}$ crossing are computed and compared with the Ansys SI Wave. The plots of computed $Z_{11}$ and $Z_{12}$ parameters are compared with the Ansys SI Wave in Figures 5.13 and 5.14, respectively. As can be seen, the results from both methods match reasonably well. Similar results with comparable accuracy were observed for other $Z$-parameter matrix elements such as $Z_{13}, Z_{14}$, and $Z_{21}$, etc. 


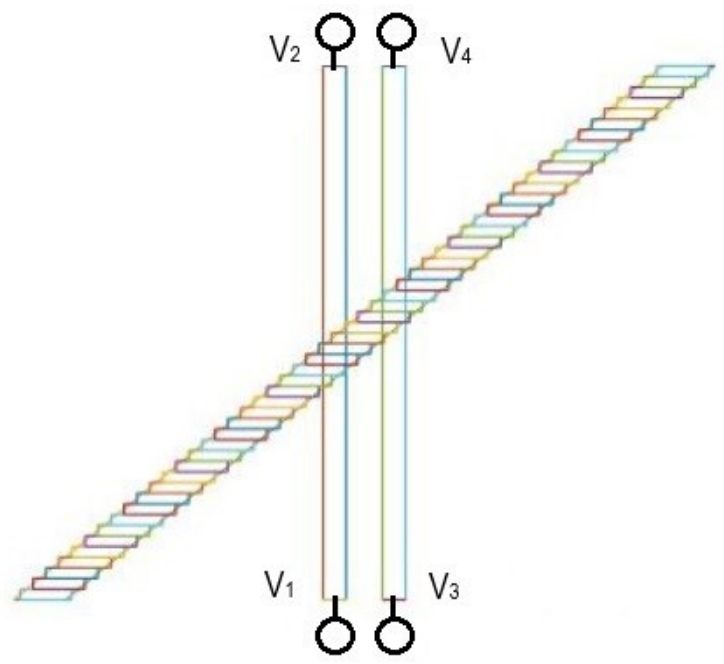

Figure 5.12: Experimental Setup with ASL with 1 Trace Crossing at $45^{\circ}$ Angle (Experiment 1) 


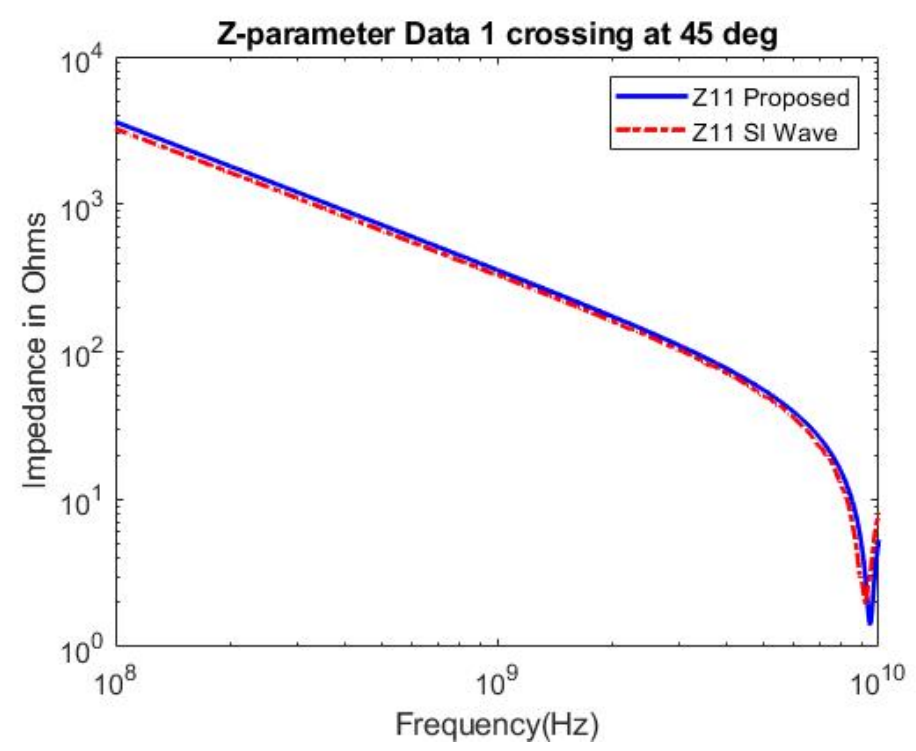

Figure 5.13: Comparison of $Z_{11}$ Parameter of the ASL with 1 Trace Crossing at $45^{\circ}$ Angle (Experiment 1)

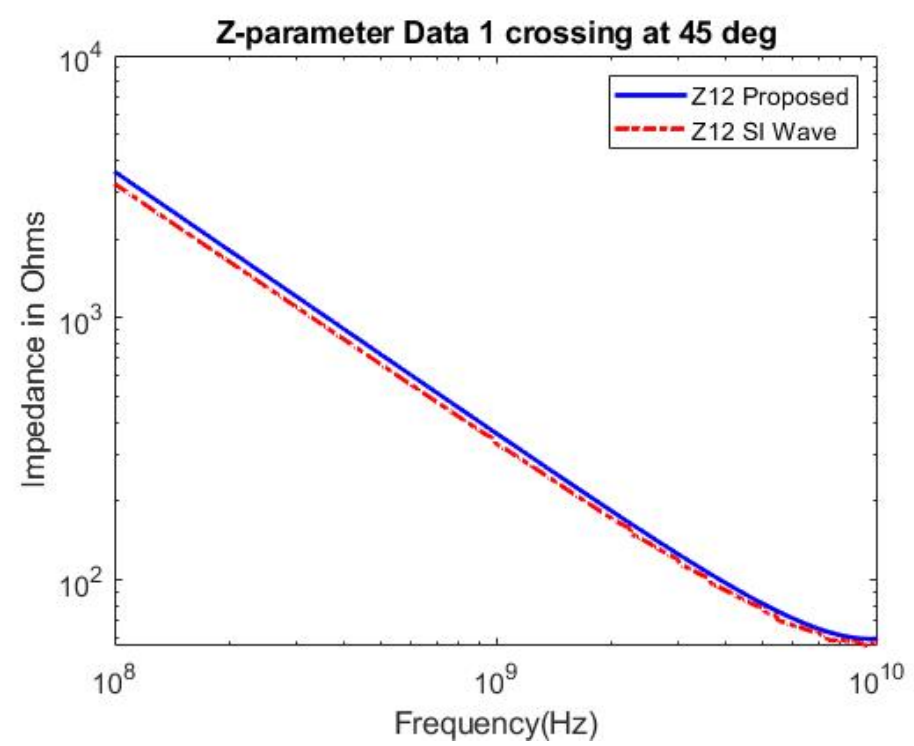

Figure 5.14: Comparison of $Z_{12}$ Parameter of the ASL with 1 Trace Crossing at $45^{\circ}$ Angle (Experiment 1) 


\section{Experiment 2: ASL with 51 Crossing Junctions at $45^{\circ}$.}

In this experiment, the proposed model for ASL is investigated in the presence of 51 equally-spaced crossing junctions at $45^{\circ}$. The setup for this experiment is shown in Figure 5.15. The $Z$ parameters for the transmission line with 51 crossings were obtained by multiplying the chain parameters for the transmission line with one crossing junction from the previous experiment 50 times and transforming the resulting chain parameters to $Z$ parameters. The $Z_{11}$ and $Z_{12}$ parameters using the proposed method are compared with the Ansys SI Wave in Figures 5.16 and 5.17, respectively. As can be seen from the comparison plots, the computed and simulated plots are in good agreement, further validating the proposed model. 


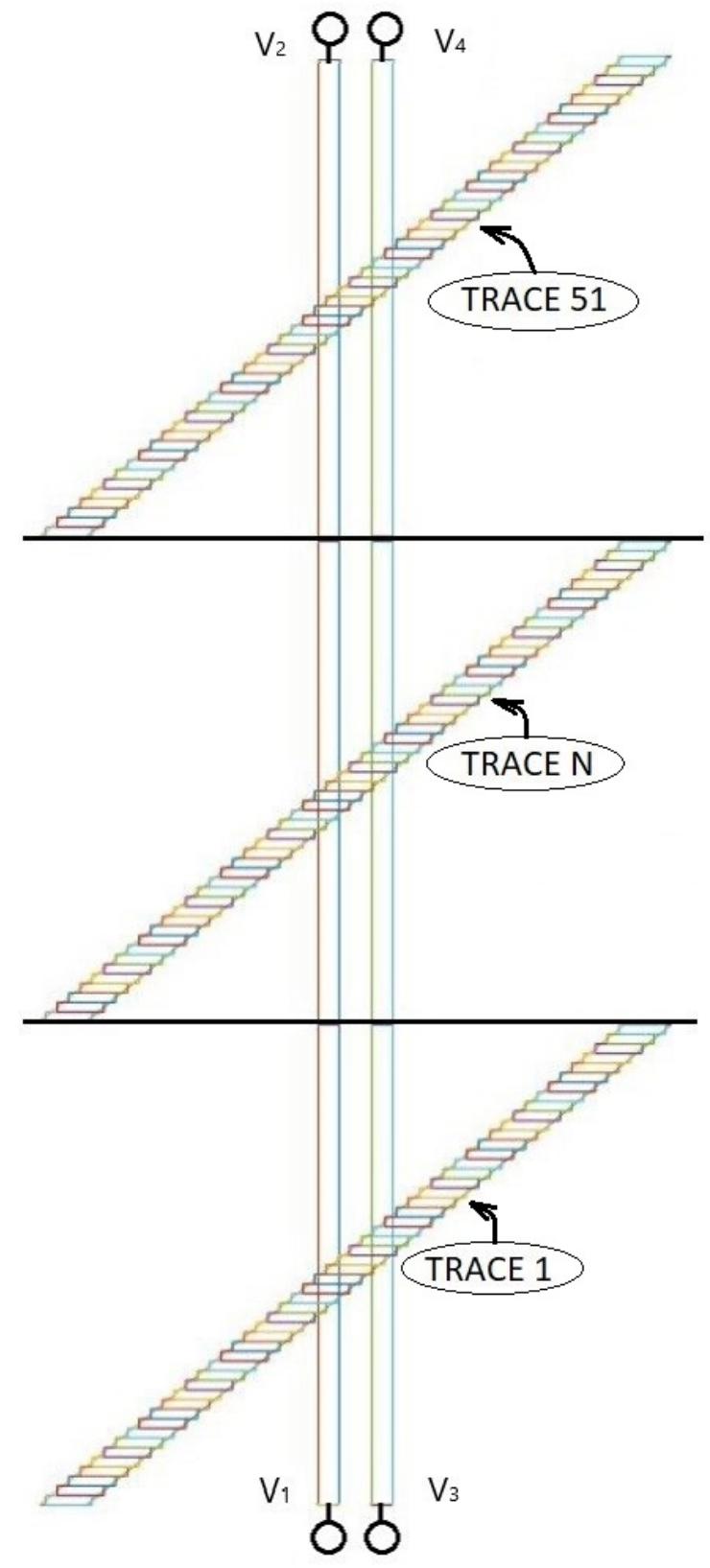

Figure 5.15: Experimental Setup with ASL with 51 Trace Crossings at $45^{\circ}$ Angle (Experiment 2) 


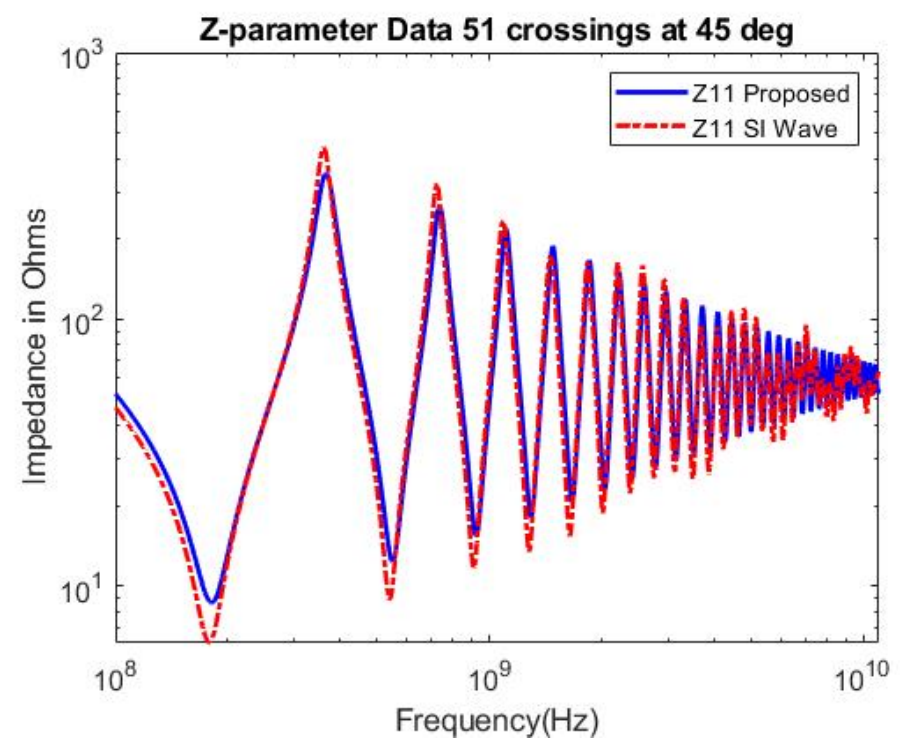

Figure 5.16: Comparison of $Z_{11}$ Parameter of the ASL with 51 Trace Crossings at $45^{\circ}$ Angle (Experiment 2)

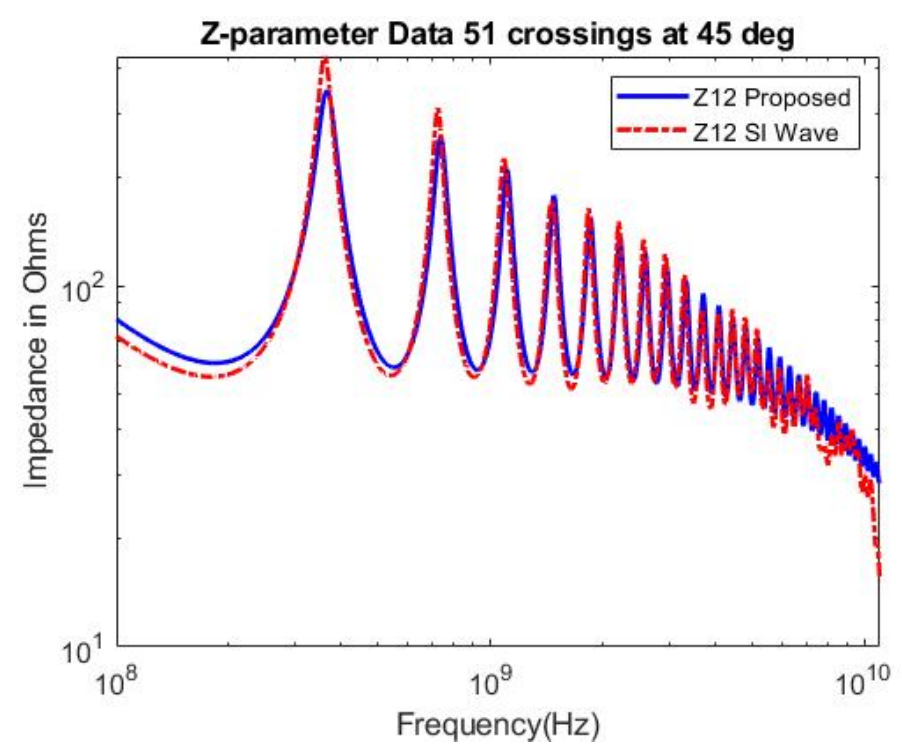

Figure 5.17: Comparison of $Z_{12}$ Parameter of the ASL with 51 Trace Crossings at $45^{\circ}$ Angle (Experiment 2) 


\section{Experiment 3: ASL with 51 Crossing Junctions at $90^{\circ}$.}

For this experiment, the differential stripline transmission line with total length of $7.5^{\prime \prime}$ with 51 equally-spaced crossing traces at $90^{\circ}$ angle was considered. The setup for this experiment is shown in Figure 5.18. The $Z$ parameters for the differential stripline in the presence of 51 crossings at $90^{\circ}$ are computed and compared with the Ansys SI Wave. The $Z_{11}$ and $Z_{12}$ parameters using the proposed method are compared with the Ansys SI Wave in Figures 5.19 and 5.20, respectively. As can be seen from the comparison plots, the results from both methods match reasonably well. 


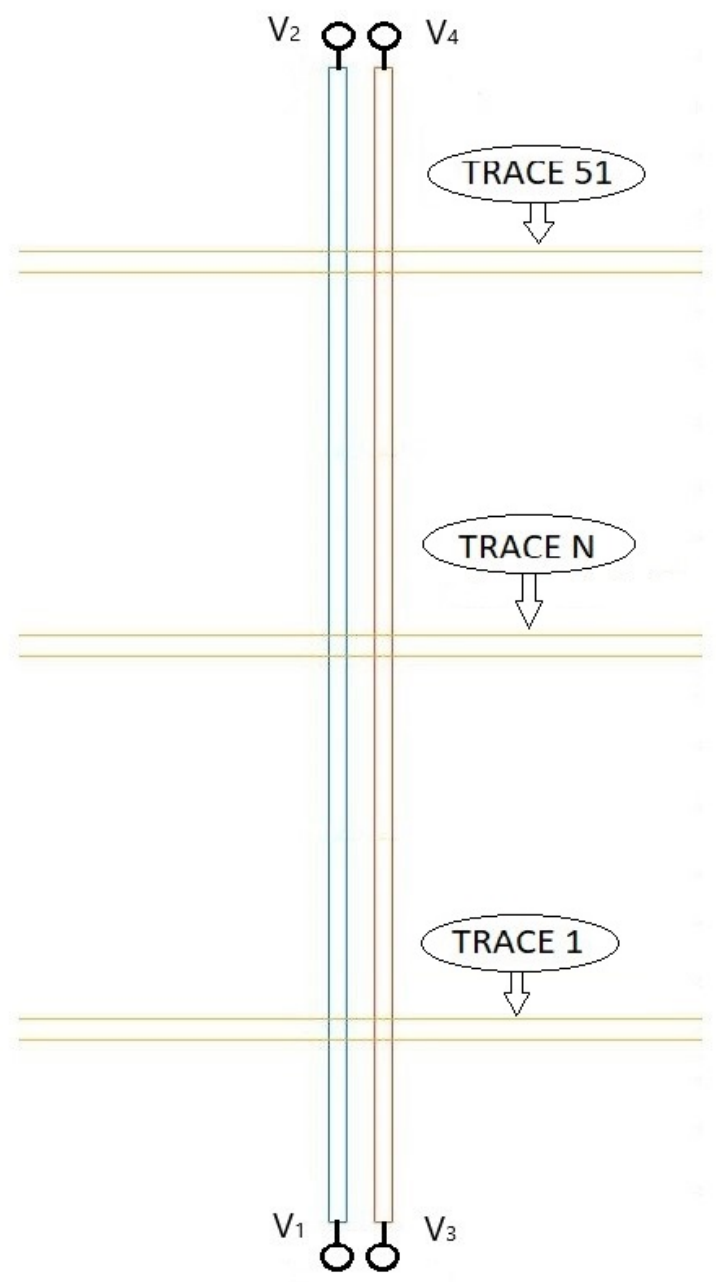

Figure 5.18: Experimental Setup with ASL with 51 Trace Crossings at $90^{\circ}$ Angle (Experiment 3) 


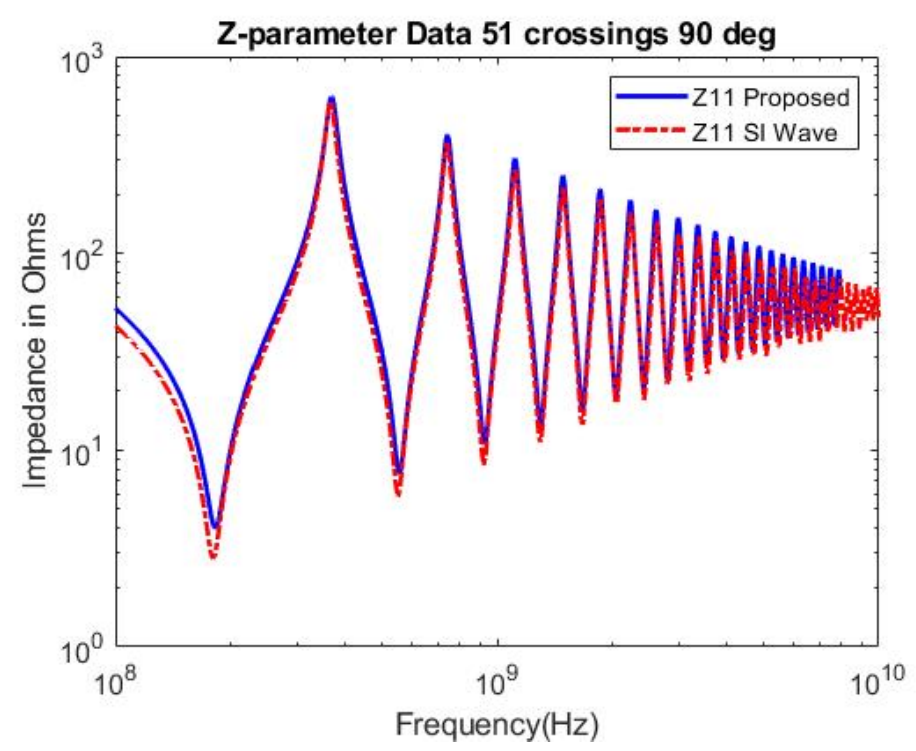

Figure 5.19: Comparison of $Z_{11}$ Parameter of the ASL with 51 Trace Crossings at $90^{\circ}$ Angle (Experiment 3 )

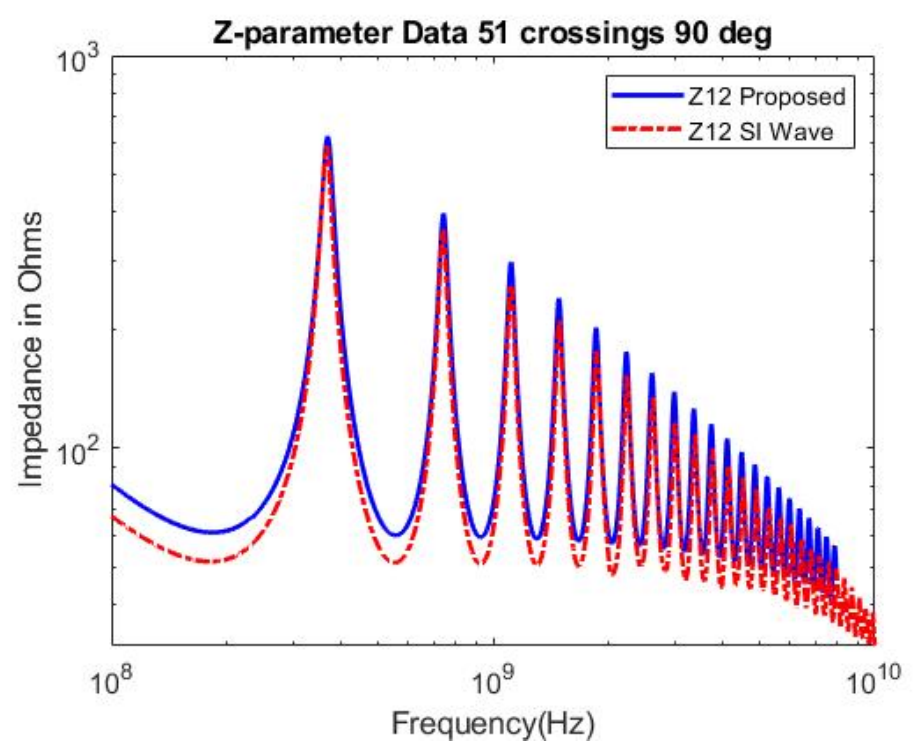

Figure 5.20: Comparison of $Z_{12}$ Parameter of the ASL with 51 Trace Crossings at $90^{\circ}$ Angle (Experiment 3) 


\section{Experiment 4. ASL with $45^{\circ}, 90^{\circ}$, and no crossings.}

In this experiment, a relatively large and mixed configuration is considered. The hybrid structure consists of ASLs with no crossing traces, with $45^{\circ}$ crossing traces and also with $90^{\circ}$ crossing traces. The setup for this experiment is shown in Figure 5.21. Figures 5.22 - 5.23 show the comparison of $Z$ parameters for 1 crossing trace. The figures show that the difference between the $Z$ parameters is negligible, so the effect of 1 crossing trace on $Z$ parameters is very small. Figures $5.24-5.25$ show the comparison of $Z$ parameters for transmission line with length of $7.5^{\prime \prime}$ with 51 equally-spaced crossing traces. Figures 5.26 - 5.27 show the comparison of $Z$ parameters for the transmission line with length of 7.5" with 102 equally-spaced crossing traces. The plots indicate that at higher frequencies, $Z$ parameters for the case with large number of crossings differ significantly from those with no crossings or less number of crossings. 


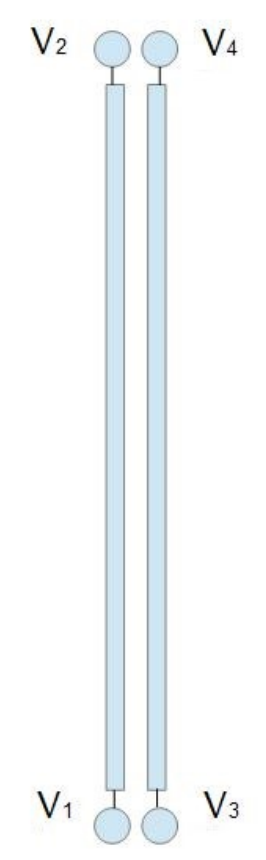

(a) No crossings

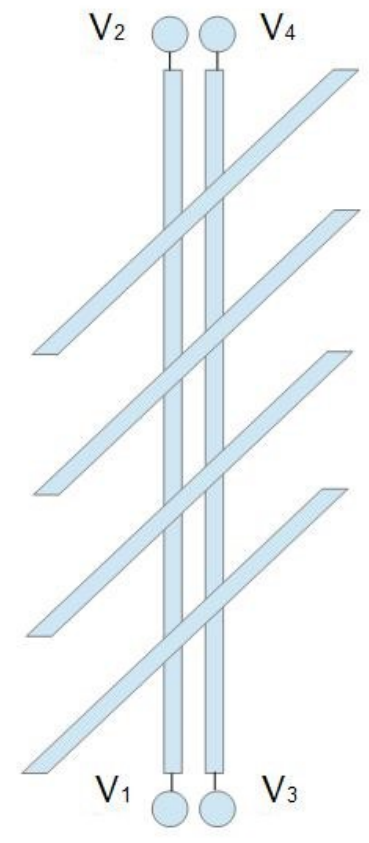

(b) $45^{\circ}$ crossings

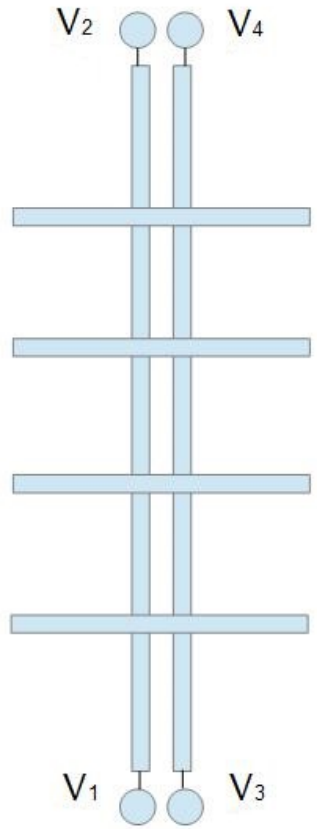

(c) $90^{\circ}$ crossings

Figure 5.21: Experimental Setup for Comparison of ASLs with No Crossings, with $45^{\circ}$ Crossings and $90^{\circ}$ Crossings (Experiment 4) 


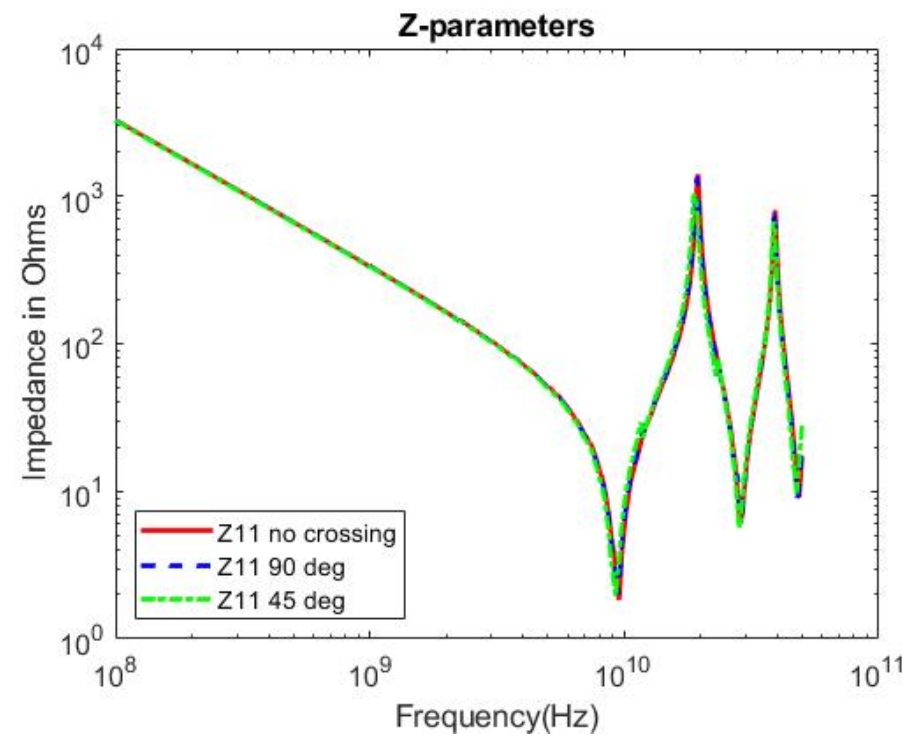

Figure 5.22: Comparison of $Z_{11}$ Parameter of the ASL with 1 Trace Crossing (Experiment 4)

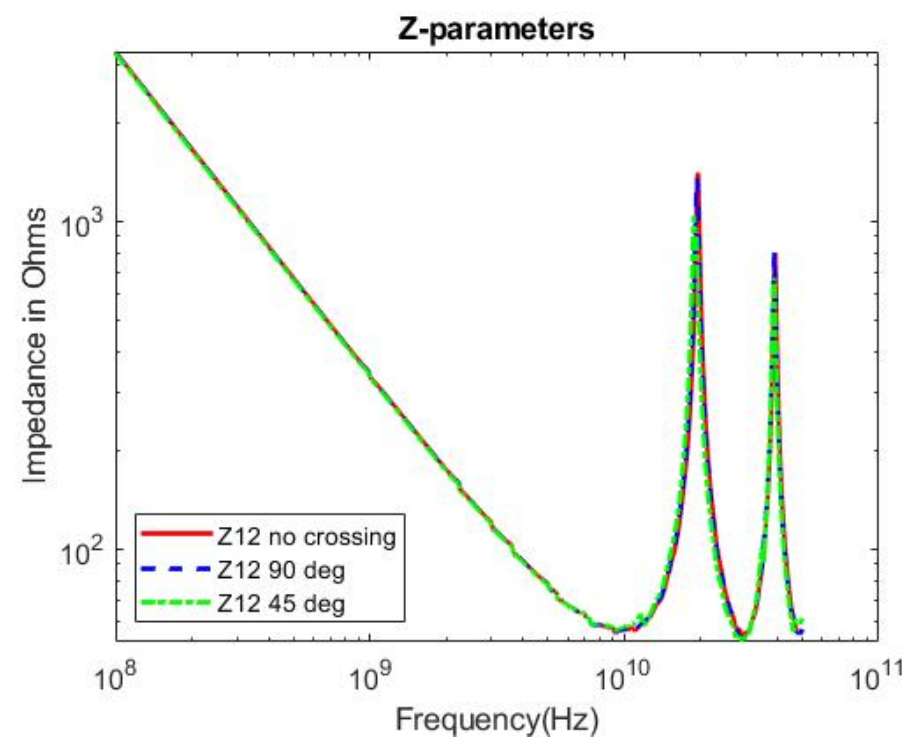

Figure 5.23: Comparison of $Z_{12}$ Parameter of the ASL with 1 Trace Crossing (Experiment 4) 


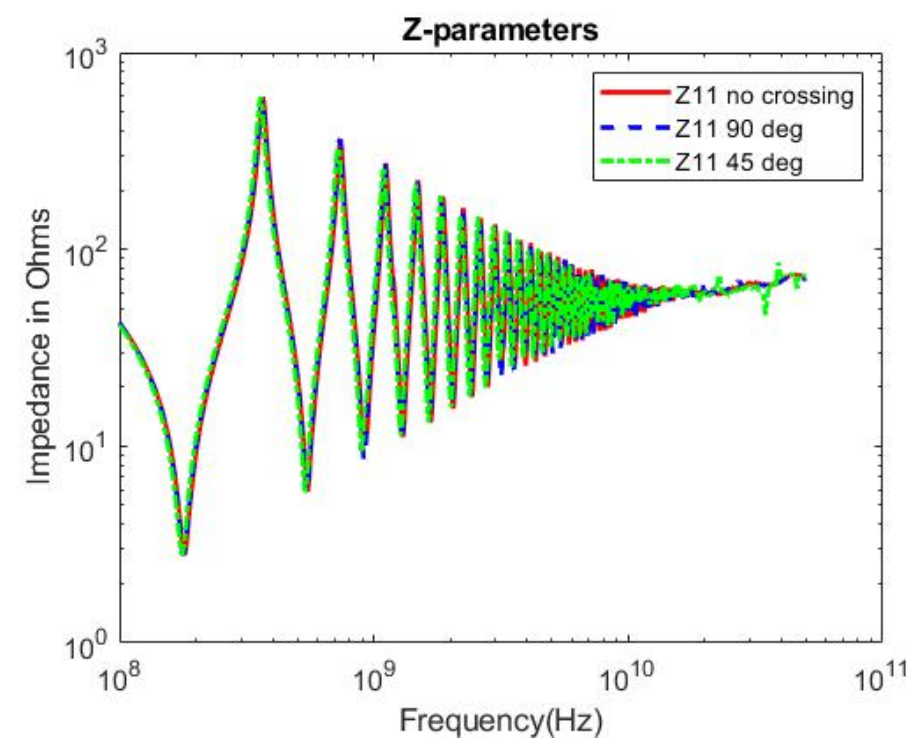

Figure 5.24: Comparison of $Z_{11}$ Parameter of the ASL with 51 Trace Crossings (Experiment 4)

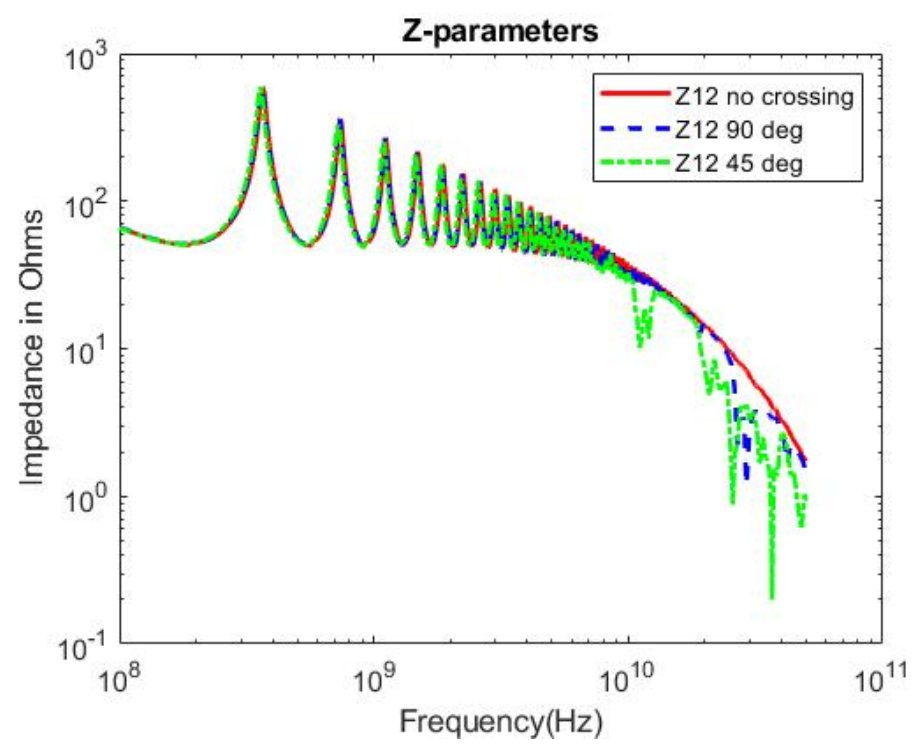

Figure 5.25: Comparison of $Z_{12}$ Parameter of the ASL with 51 Trace Crossings (Experiment 4) 


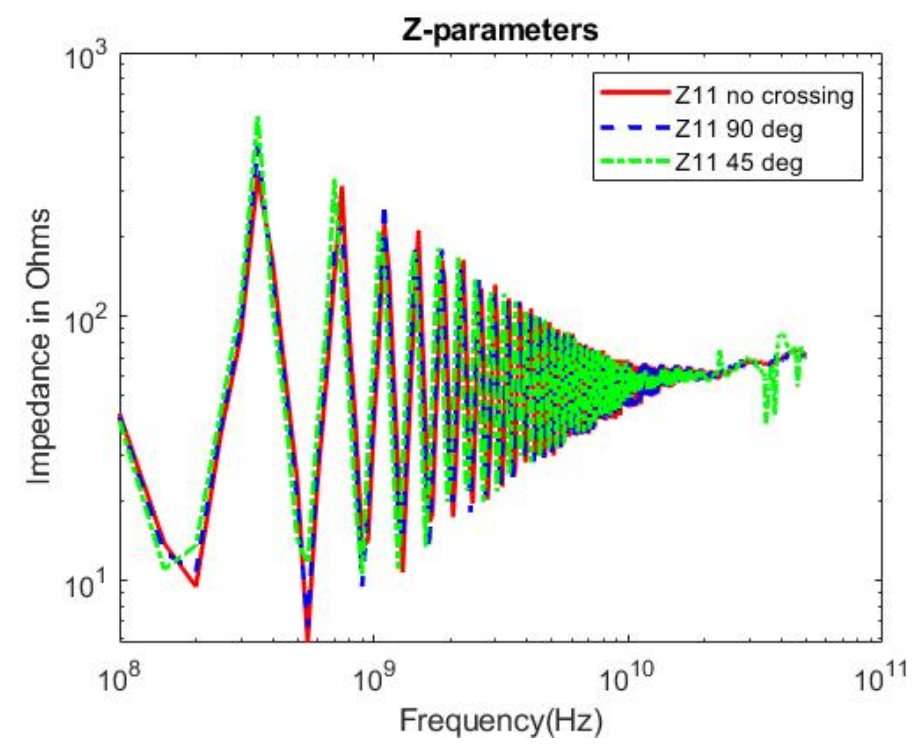

Figure 5.26: Comparison of $Z_{11}$ Parameter of the ASL with 102 Trace Crossings (Experiment 4)

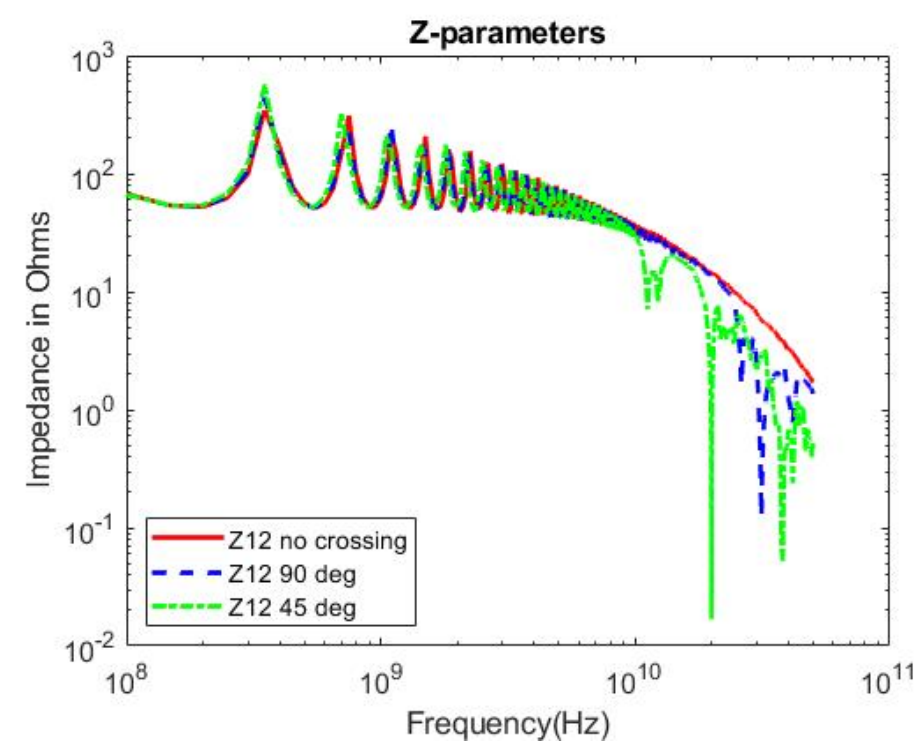

Figure 5.27: Comparison of $Z_{12}$ Parameter of the ASL with 102 Trace Crossings (Experiment 4) 


\subsection{An Investigation of Impact of Crossing Junctions at Various Angles on Signal In- tegrity Performance}

In the following experiments, the effect of discontinuities of crossing junctions on signal integrity is demonstrated for the PCIe serial link at 16 Gbps for differential stripline with 102 equally-spaced crossing traces at $45^{\circ}, 90^{\circ}$ and no crossings with an ASL of length 7.5" (as described in Figure 5.21). Four experiments were performed based on different ASL cross-section dimensions defined in Figure 5.28 and given in Table 5.9 as will become evident in the results.

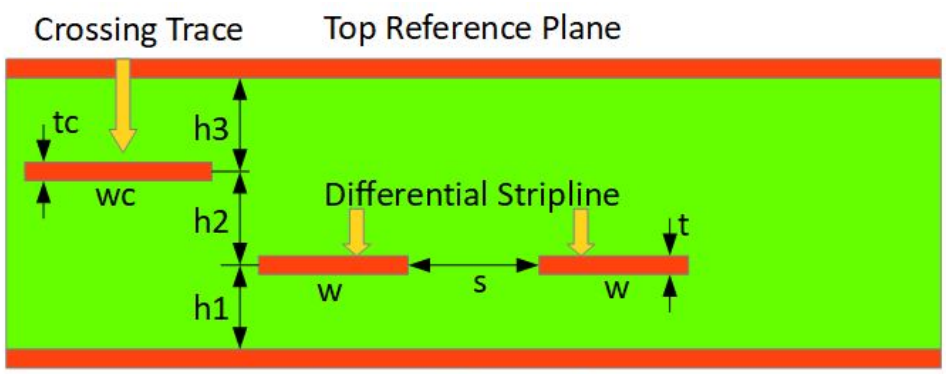

Bottom Reference Plane

Figure 5.28: ASL Cross-section Dimension Definitions 
Table 5.9: Cross-section Dimensions (in mils) for the Experiments 5-8

\begin{tabular}{c|c|c|c|c|c|c}
\hline \hline Parameter & h1 & h2 & h3 & w & s & t \\
\hline Experiment 5 & 5 & 5 & 5 & 5 & 8 & 0.7 \\
Experiment 6 & 10 & 5 & 10 & 5 & 8 & 0.7 \\
Experiment 7 & 3 & 3 & 3 & 5 & 8 & 0.7 \\
Experiment 8 & 3 & 3 & 3 & 5 & 4 & 0.7 \\
\hline
\end{tabular}


Experiment 5: $h 1=h 2=h 3=5 \mathrm{mils}, s=8 \mathrm{mils}$.

The resulting eye diagrams for this experiment are shown in Figures 5.29

- 5.31. The eye diagram measurements are shown in Table 5.10. For this example, results for the case of $45^{\circ}$ provide degradation in eye height and width compared to the case of no crossings or $90^{\circ}$ crossings. Also, the jitter in the case of $45^{\circ}$ crossings is higher compared to the case of no crossings or $90^{\circ}$ crossings.

Table 5.10: Eye Diagram Measurements

\begin{tabular}{cccc}
\hline \hline Parameter & No Crossings & $90^{\circ}$ Crossings & $45^{\circ}$ Crossings \\
\hline Eye Height, mV & 245.87 & 244.18 & 230.14 \\
Eye Width, ps & 44.88 & 44.13 & 43.5 \\
Jitter, p2p, ps & 18.25 & 19.13 & 19.75 \\
\hline
\end{tabular}

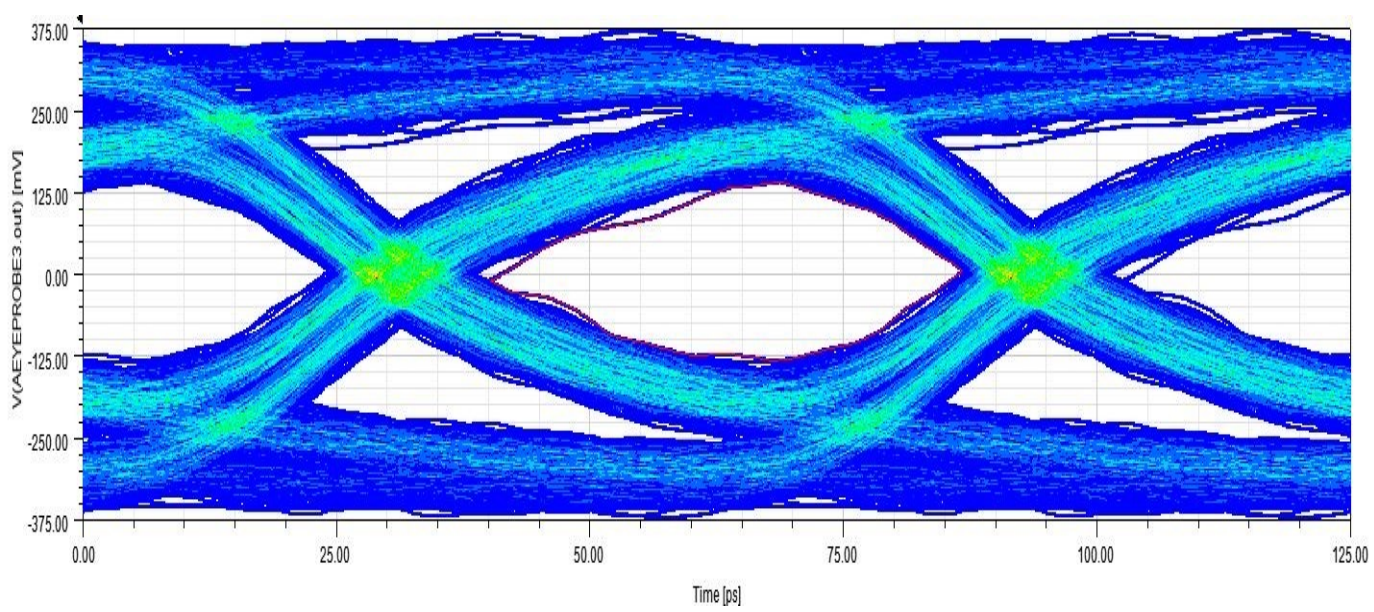

Figure 5.29: Eye Diagram for 7.5" ASL with No Crossings (Experiment 5) 


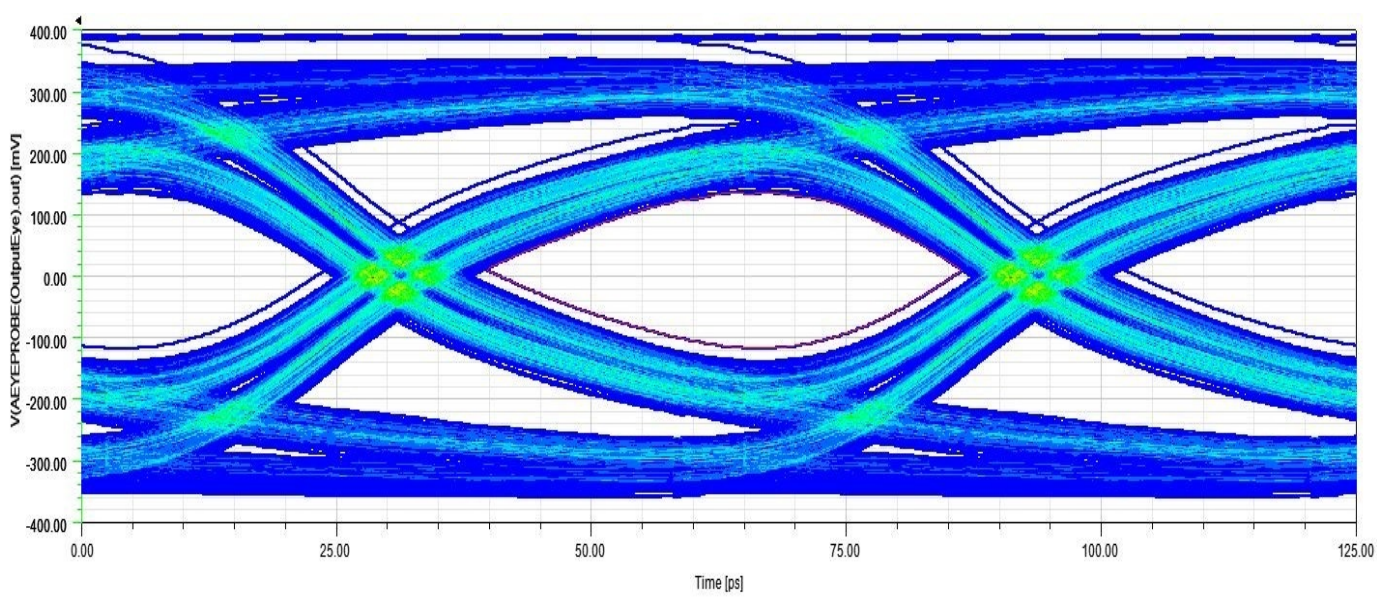

Figure 5.30: Eye Diagram for 7.5" ASL with 102 Crossings at $90^{\circ}$ angle (Experiment 5)

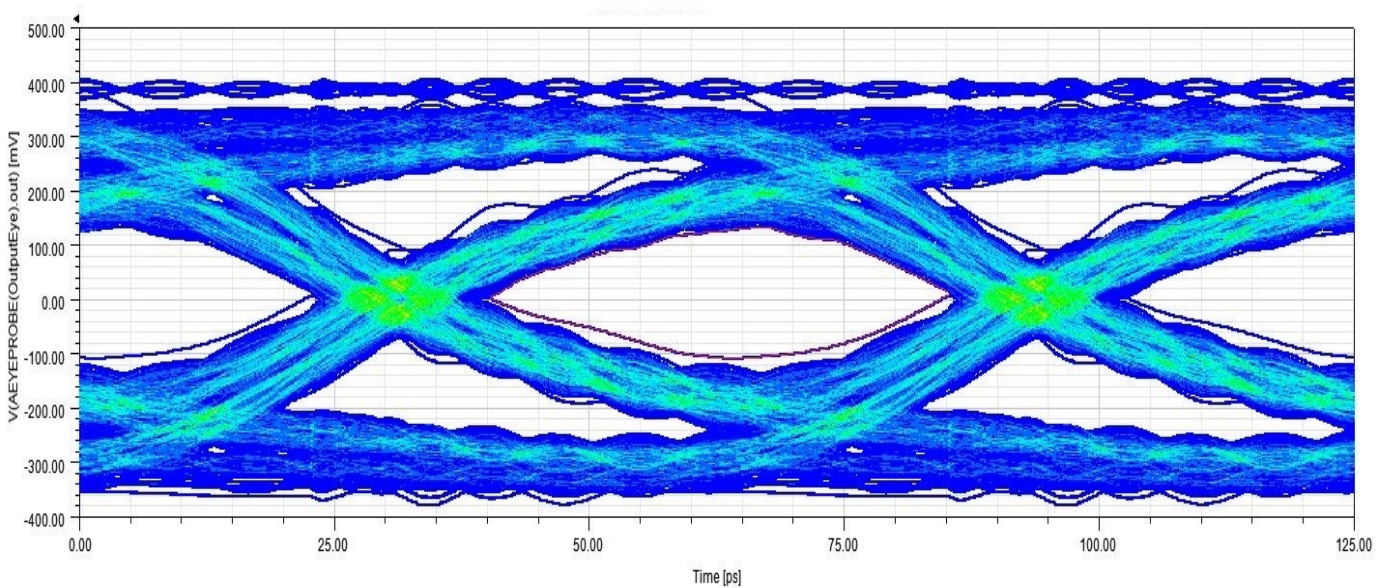

Figure 5.31: Eye Diagram for 7.5" ASL with 102 Crossings at $45^{\circ}$ angle (Experiment 5) 
Experiment 6: $h 1=h 3=10 \mathrm{mils}, \mathrm{h} 2=5 \mathrm{mils}, \mathrm{s}=8 \mathrm{mils}$ (increased distance between the reference planes and the signal conductors).

The eye diagram measurements for this experiment are shown in Table 5.11. As can be seen from the table, in this experiment too, the case of $45^{\circ}$ crossing performed inferiorly compared to both other cases.

Table 5.11: Eye Diagram Measurements

\begin{tabular}{cccc}
\hline \hline Parameter & No Crossings & $90^{\circ}$ Crossings & $45^{\circ}$ Crossings \\
\hline Eye Height, mV & 266.79 & 241.17 & 219.22 \\
Eye Width, ps & 47.13 & 45.0 & 43.75 \\
Jitter, p2p, ps & 16.13 & 18.25 & 19.63 \\
\hline
\end{tabular}


Experiment 7: $h 1=h 2=h 3=3$ mils, $s=8$ mils (decreased distance between the reference planes and the signal conductors as well as decreased distance between the crossing conductor and the differential stripline).

The eye diagram measurements for this experiment are shown in Table 5.12. In this example too, results show a significant degradation in the eye diagram for the cases with crossings compared to the case without crossings. In this experiment too, the case with $45^{\circ}$ oblique crossing performed poorly compared to both other cases. Such an information needs to be taken into account in the timing budget of high-speed designs while designing the layouts.

Table 5.12: Eye Diagram Measurements

\begin{tabular}{cccc}
\hline \hline Parameter & No Crossings & $90^{\circ}$ Crossings & $45^{\circ}$ Crossings \\
\hline Eye Height, mV & 195.6 & 190.74 & 166.92 \\
Eye Width, ps & 36.13 & 37.13 & 34 \\
Jitter, p2p, ps & 27.25 & 26.38 & 29.5 \\
\hline
\end{tabular}


Experiment 8: h1=h2=h3=3mils, $s=4$ mils (decreased distance between reference planes and signal conductors, decreased distance between the crossing conductor and the differential stripline as well as decreased distance between the conductors of differential stripline.

The resulting eye diagrams for this experiment are shown in Figures 5.32 - 5.34. The eye diagram measurements are shown in Table 5.13. In this example too, results show a significant degradation in the eye diagram for the cases with crossings compared to the case without crossings. In this experiment, the case with $90^{\circ}$ oblique crossing performed poorly compared to both other cases. Such an information needs to be taken into account in the timing budget of high-speed designs while designing the layouts.

Table 5.13: Eye Diagram Measurements

\begin{tabular}{cccc}
\hline \hline Parameter & No Crossings & $90^{\circ}$ Crossings & $45^{\circ}$ Crossings \\
\hline Eye Height, mV & 178.65 & 100.74 & 162.66 \\
Eye Width, ps & 33.63 & 29.5 & 31.7 \\
Jitter, p2p, ps & 30.0 & 34.13 & 31.75 \\
\hline
\end{tabular}




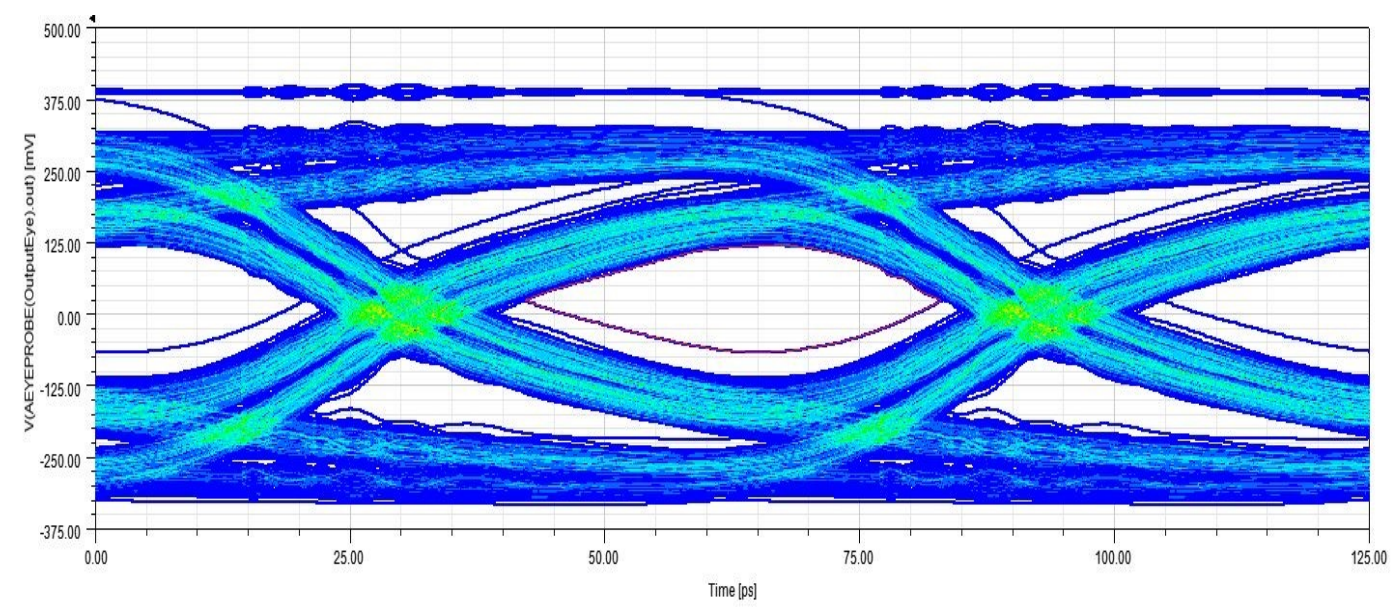

Figure 5.32: Eye Diagram for 7.5" ASL with No Crossings (Experiment 8)

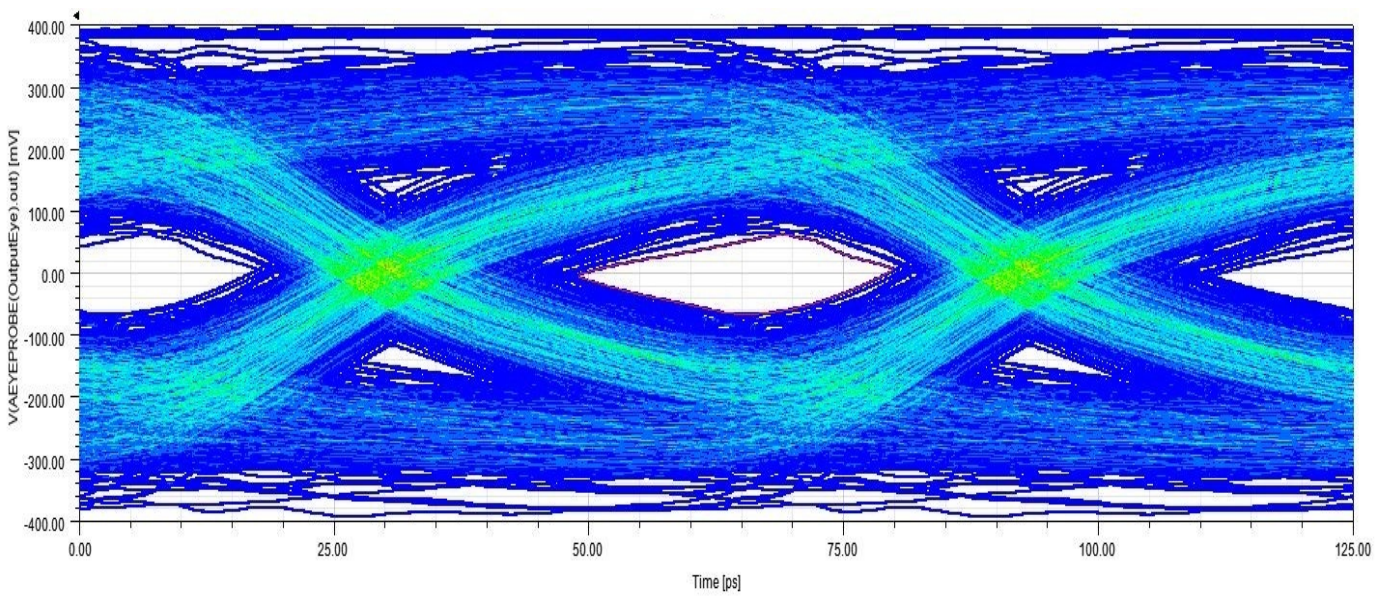

Figure 5.33: Eye Diagram for 7.5" ASL with 102 Crossings at $90^{\circ}$ angle (Experiment 8) 


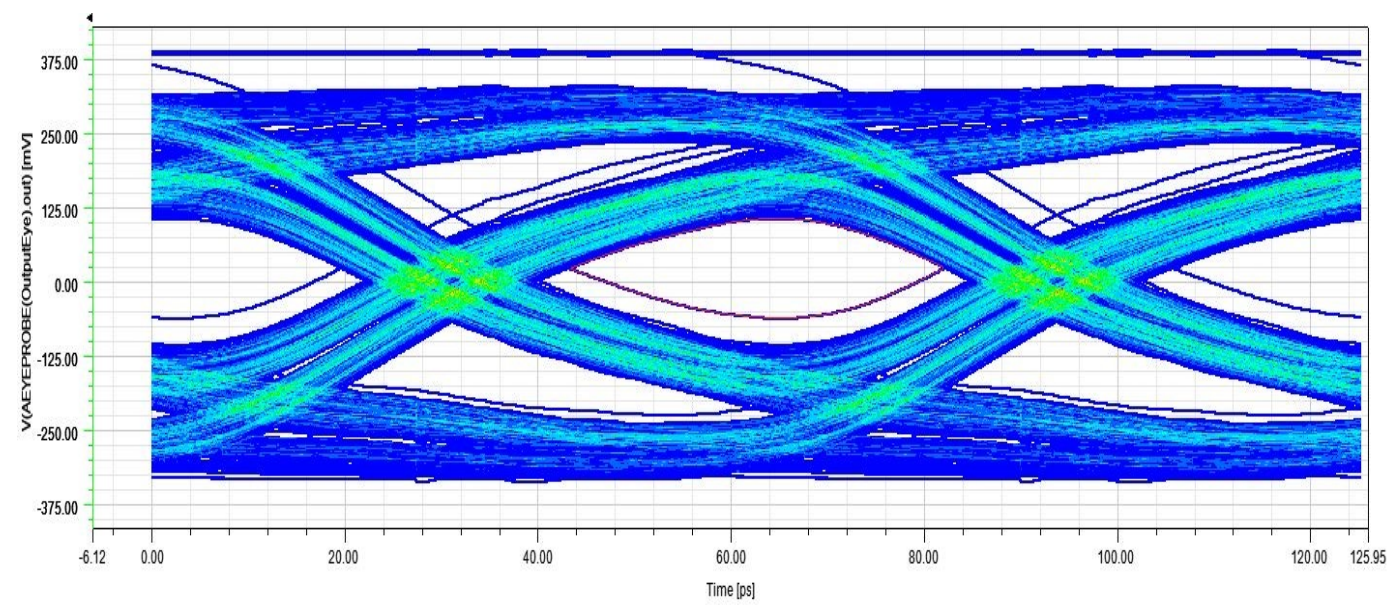

Figure 5.34: Eye Diagram for 7.5" ASL with 102 Crossings at $45^{\circ}$ angle (Experiment 8) 


\section{Chapter 6}

\section{Conclusion}

In this thesis, modeling of differential stripline with crossing trace junctions has been advanced. Particularly,

(a) an efficient model for differential asymmetrical striplines with oblique crossing junctions has been developed.

(b) effect of the crossing trace angle on the signal integrity metrics has been investigated.

The results of the analysis were compared to the commercial EM and signal integrity tools and showed good matching, validating the proposed solutions. A study of the effect of crossing traces on the performance of the differential stripline transmission line was conducted. It was shown for some ASL crosssections the effect of crossing traces on the eye diagram is significant and should be taken into account when designing high-speed differential transmission lines. This is particularly the case when the distance between the crossing traces and the differential stripline is reduced or when the distance 
between the signal traces in differential stripline is reduced.

Future Work: Future research in this area can be done based on the work in this thesis to develop:

(a) An alternative efficient approach based on Schwarz-Christoffel transformation for modeling of ASL with oblique crossings.

(b) Sensitivity analysis of high-speed systems with ASLs having oblique crossings.

(c) Use of the proposed model for optimization of signal integrity metrics. 


\section{Bibliography}

[1] D. K. Cheng, Field and Wave Electromagnetics, MA: Addison-Wesley, 2nd edition, 1989.

[2] H. Johnson and M. Graham, High-Speed Digital Design, NJ: Prentice Hall, 1993.

[3] D. Brooks, Signal Integrity Issues and Printed Circuit Board Design, NJ: Prentice Hall, 2003.

[4] E. Bogatin, Signal Integrity - Simplified, MA: Prentice Hall, 2004.

[5] C. Nguyen, Analysis Methods For RF, Microwave, And Millimeter-Wave Planar Transmission Line Structures, NY: John Wiley \& Sons, 2000.

[6] I. Erdin and R. Achar, "Characteristic impedance of asymmetrical differential traces: Closed-form relations for signal integrity analysis," Proceedings of IEEE Electrical Design of Advanced Packaging and Systems Symposium, Seoul, SouthKorea, Dec. 2015, pp. 43-45.

[7] I. Erdin and R. Achar, "Modeling of Asymmetric Differential Striplines Including Crossing Junction Discontinuities," IEEE Transactions on 
Electromagnetic Compatibility, Volume: 60, Issue: 1, Feb. 2018, pp. 5058.

[8] C. R. Paul, Analysis of Multiconductor Transmission Lines, NY: John Wiley \& Sons, 2nd edition, 2008.

[9] D. A. McNamara, Topics in Electromagnetics I: Moment Method (in Engineering Electromagnetics), School of Electrical Engineering \& Computer Science, Faculty of Engineering, University of Ottawa, Winter 2018.

[10] C. R. Paul, Introduction to Electromagnetic Compatibility, NY: John Wiley \& Sons, 2nd edition, 2006.

[11] K. Ogata, State Space Analysis of Control Systems, Prentice-Hall, Englewood Cliffs, NJ, 1967.

[12] C. T. Chen, Linear System Theory and Design, Holt, Rinehart and Winston, New York, 1984.

[13] F. E. Hohn, Elementary Matrix Algebra, 2nd edition, Macmillan, New York, 1964.

[14] A. Ralston, A First Course in Numerical Analysis, McGraw-Hill, New York, 1965.

[15] V. Tripathi, "Asymmetric coupled transmission lines in an inhomogeneous medium," IEEE Trans. Microw. Theory Techn., vol. MTT-23, no. 9, pp. 734-739, Sep. 1975. 
[16] E. G. Crystal, "Coupled-transmission-line directional couplers with coupled lines of unequal characteristic impedance," IEEE Trans. Microw. Theory Techn., vol. MTT-14, pp. 337-346, July 1966.

[17] Q. Gu and J. A. Kong, "Transient analysis of single and coupled lines with capacitively loaded junctions," IEEE Trans. Microw. Theory Techn., vol. MTT-34, no. 9, pp. 952-964, Sep. 1986.

[18] K. S.Olsen,G.W. Pan, and B. K. Gilbert, "Comments on transient analysis of single and coupled lines with capacitively loaded junctions," IEEE Trans. Microw. Theory Techn., vol. MTT-35, no. 10, pp. 929-930, Sep. 1987.

[19] G. W. Pan, K. S. Olsen, and B. K. Gilbert, "Frequency domain solution for coupled striplines with crossing strips," IEEE Trans. Microw. Theory Techn., vol. 39, no. 6, pp. 1013-1017, Jun. 1991.

[20] G. W. Pan, K. S. Olsen, and B. K. Gilbert, "Improved algorithmic methods for the prediction of wavefront propagation behavior in multiconductor transmission lines for high frequency digital signal processors," IEEE Trans. Comput.-Aided Des. Integr. Circuits Syst., vol. 8, no. 6, pp. 608-621, Jun. 1989.

[21] A. K. Hamid and M. Zu, "Modeling and simulation of crossing striplines in printed circuit boards or multichip modules," in Proc. 1992 Symp. Antenna Technol. Appl. Electromagn., Winnipeg, MB, Canada, Jul. 1992, pp. 391-396. 
[22] T. Uwano, R. Sorrentino, and T. Itoh, "Characterization of stripline crossing by transverse resonance analysis," IEEE Trans.Microw. Theory Techn., vol. MTT-35, no. 12, pp. 1369-1376, Dec. 1987.

[23] W. Veit, H. Diestel, and R. Pregla, "Coupling of crossed planar multiconductor systems," IEEE Trans. Microw. Theory Techn., vol. 38, no. 3, pp. 265-269, Mar. 1990.

[24] M. Nakhla and Q. J. Zhang, Eds., Modeling and Simulation of HighSpeed VLSI Interconnects, Boston, MA, USA: Kluwer, 1994.

[25] T.-S. Horng, "A rigorous study of microstrip crossovers and their possible improvements," IEEE Trans. Microw. Theory Techn., vol. 42, no. 9, pp. 1802-1806, Sep. 1994.

[26] T. C. Edwards and M. B. Steer, Foundations for Microstrip Circuit Design, 4th ed. Hoboken, NJ, USA: Wiley, 2016.

[27] Y. Kami and R. Sato, "Coupling model of crossing transmission lines," IEEE Trans. Electromagn. Compat., vol. EMC-28, no. 4, pp. 204-210, Nov. 1986. 
Appendix A

Matlab Code for Calculation of

Per-Unit-Length Capacitance

Matrices 
\% version: 2.0

\% created: March 4, 2018

\% Revised: Feb 16, 2020

\% Description: oblique Crossing of Dual stripline

\% Author: Peter Bliznyuk-Kvitko

clear a11;

close a11;

clc;

\% Define the structure parameters

$w=5 ; \%$ width of dual stripline conductor in mils

$\mathrm{s}=8 ; \%$ spacing between dual stripline conductors in mils

wc $=8 ; \%$ width of crossing conductor in mils

$\mathrm{L}=200$; \% Length of crossing conductor in mils

$\mathrm{N}=50$; \% Number of $\mathrm{Z}$ direction segments

$\%$ figure number

fnum $=1$;

$\%$ output file for the C matrix elements

fileID = fopen ('output_ccoeff.txt' , 'w');

$\%$ Generate the C matrix elements and plots for angles $0,15,30,45,60$, $\% 75$ and 90 degrees between crossing conductor and differential stripline for ang $=0: 15: 90$

fprintf("Solving Мом for \%d deg angle ... \n", ang);

$\mathrm{a}=$ degtorad(ang); \% Convert the angle from degrees to radians

\% calculate start coordinates for crossing conductor

xc1_start $=-0.5 *(L * \sin (a)+w c / \cos (a))$;

yc1_start $=-0.5 *(L * \cos (a)+w c * \sin (a))$;

xc2_start $=-0.5 *(L * \sin (a)-w c / \cos (a))$;

yc2_start $=-0.5 *(L * \cos (a)+w c * \sin (a))$;

\% Calculate end coordinates for crossing conductor

$x c 1$ end $=0.5 *(L * \sin (a)-w c / \cos (a))$;

yc1_end $=0.5 *(L * \cos (a)+w c * \sin (a))$;

$x c 2$ end $=0.5 *(L * \sin (a)+w c / \cos (a))$;

yc2_end $=0.5 *(L * \cos (a)+w c * \sin (a))$;

w2d = xc2_start - xc1_start;

if (xc2_end $>$ L/2)

$m=\tan (p i / 2-a)$;

$b=0.5 * w c / \sin (a)$;

xc1_new $=-L / 2$; 


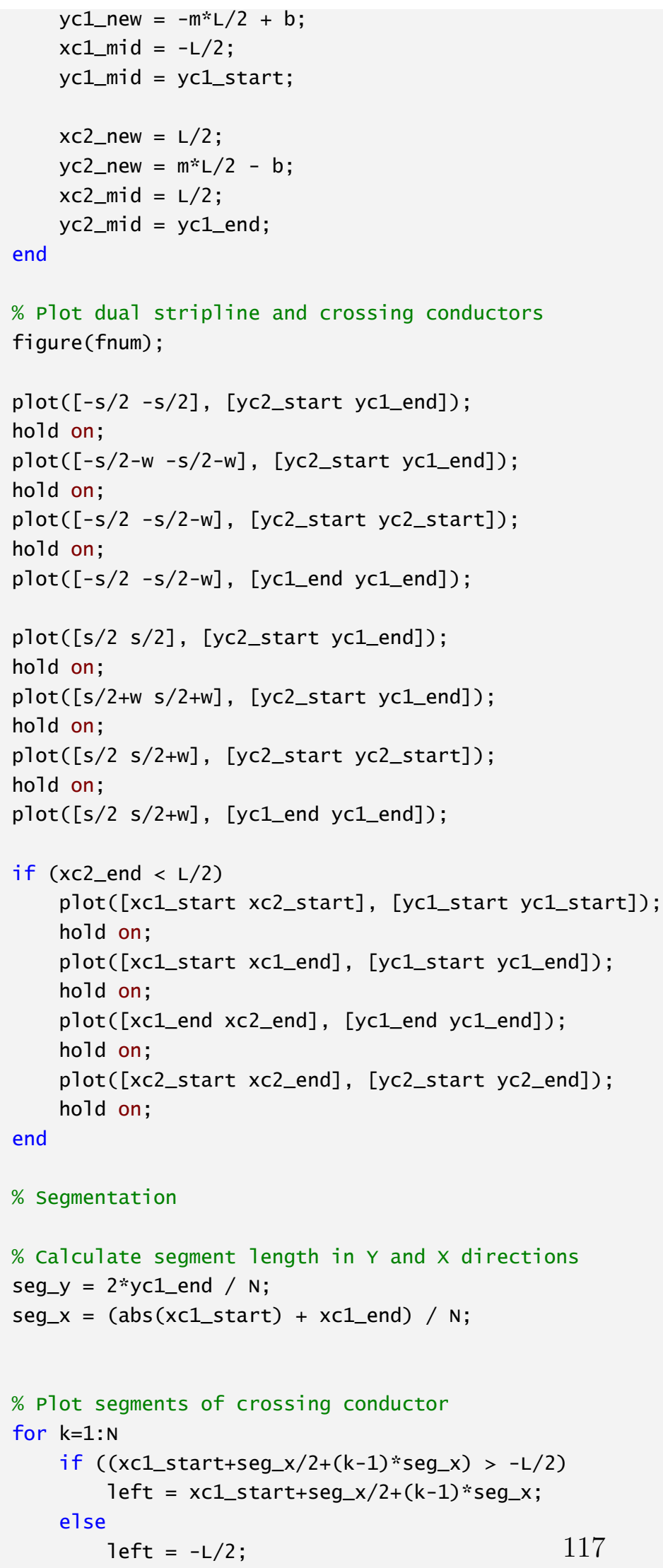




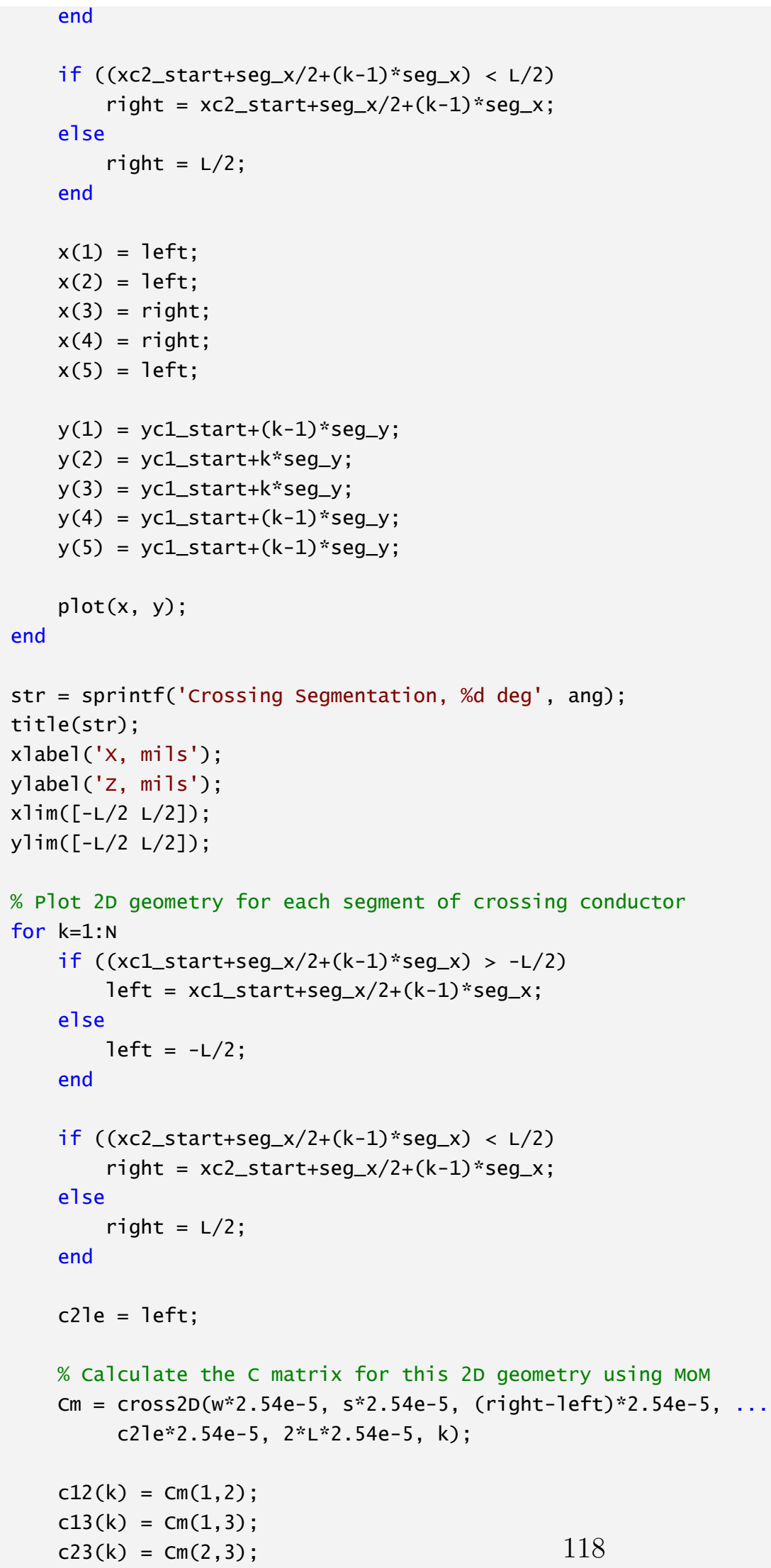




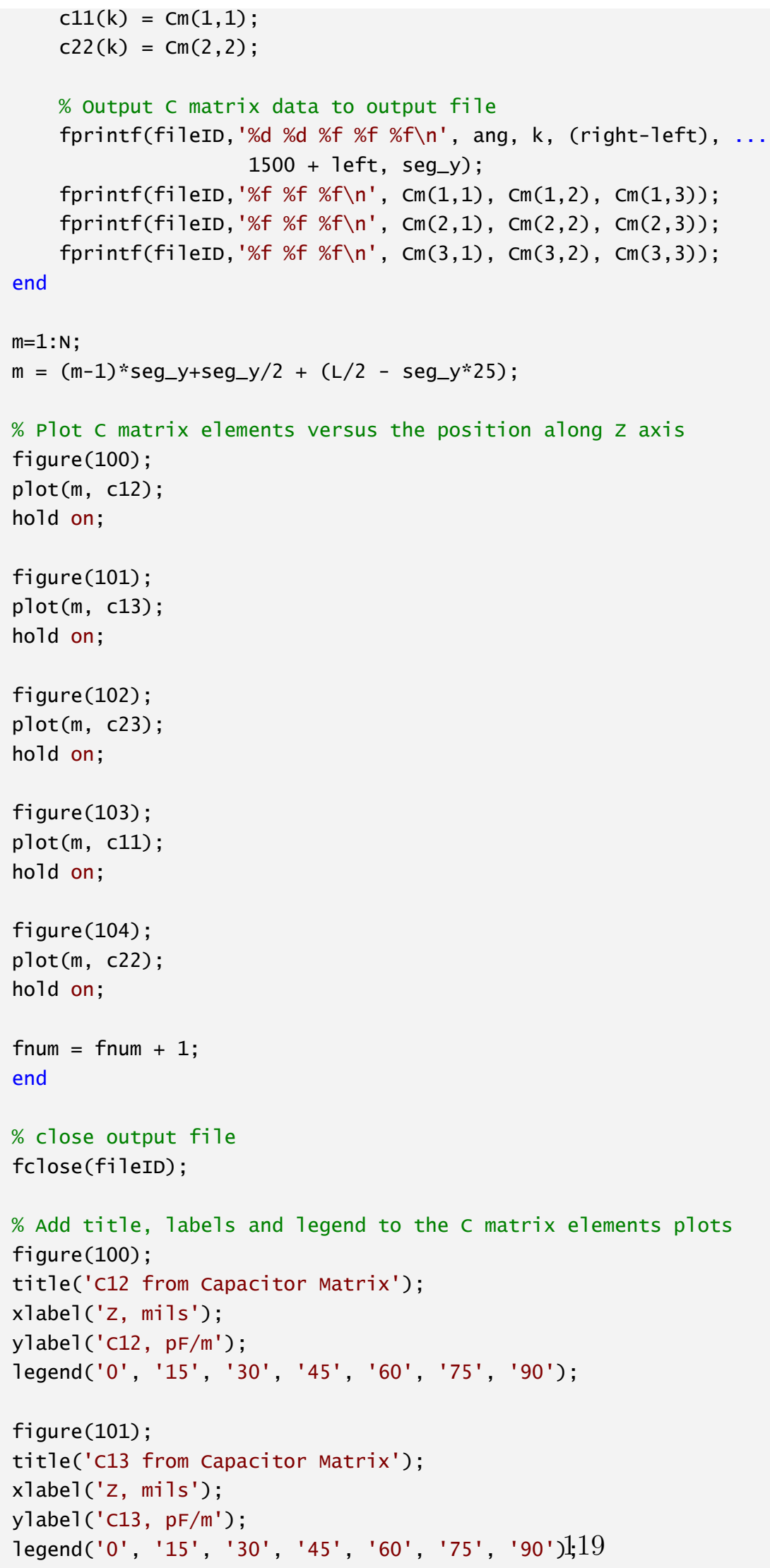


figure (102);

title('C23 from Capacitor Matrix');

xlabel('z, mils');

ylabel ('C23, pF/m');

legend('0', '15', '30', '45', '60', '75', '90');

figure(103);

title('C11 from Capacitor Matrix');

xlabe1 ('z, mils');

ylabe1 ('C11, pF/m');

legend('0', '15', '30', '45', '60', '75', '90');

figure(104);

title('c22 from Capacitor Matrix');

xlabel ('z, mils');

ylabel ('C22, pF/m') ;

legend('0', '15', '30', '45', '60', '75', '90');

Published with MATLAB ${ }^{\circledR}$ R2019b 
$\%$ version: 2.0

\% created: March 4, 2018

\% Revised: Feb 16, 2020

$\%$ Description: Method of Moments

\% Author: Peter Bliznyuk-Kvitko

\% Function cross2D

$\%$ Input parameters

$\% \quad$ w - width of dual stripline conductor in $\mathrm{m}$

$\% \quad s$ - spacing between dual stripline conductors in $\mathrm{m}$

$\%$ wc - width of the crossing conductor in XY plane in $\mathrm{m}$

$\% \quad c 21$ e - center to left edge of the crossing conductor on $x$ axis

$\%$ in $\mathrm{m}$

$\% \quad \mathrm{~L}$ - Length of the reference conductor in $\mathrm{m}$

$\% \quad k$ - slice number

$\%$ output parameter:

\% Cmatrix - Capacitance Matrix

function cmatrix $=\operatorname{cross} 2 \mathrm{D}(w, s, w c, c 21 \mathrm{e}, \mathrm{L}, \mathrm{k})$;

$\mathrm{t}=0.7 * 2.54 \mathrm{e}-5 ; \%$ thickness of dual stripline sssconductor

h1 $=5 * 2.54 \mathrm{e}-5 ; \%$ height between bottom to dual stripline conductors

$\mathrm{h} 2=5 * 2.54 \mathrm{e}-5 ; \%$ height between dual stripline and crossing conductors

h3 $=5 * 2.54 \mathrm{e}-5 ; \%$ height between crossing and top conductors

tc $=0.7 * 2.54 \mathrm{e}-5 ; \%$ thickness of crossing conductor

$\mathrm{N} 1=100 ; \%$ number of segments for top conductor

$\mathrm{N} 2=100 ; \%$ number of segments for bottom conductor

$\mathrm{N} 3=30$; $\%$ number of segments for 1eft conductor of dual stripline

$\mathrm{N} 4=30 ; \%$ number of segments for right conductor of dual stripline

$\mathrm{N} 5=100 ; \%$ number of segments for crossing conductor

$\%$ Top conductor segment coordinates

for $\mathrm{q}=1: \mathrm{N} 1$

$x t 1(q)=(q-1) * L / N 1 ;$

$z t 1(q)=h 1+t+h 2+t c+h 3 ;$

$x \operatorname{t2}(q)=q * L / N 1$

$z t 2(q)=h 1+t+h 2+t c+h 3 ;$

end

$\%$ Bottom conductor segment coordinates

for $q=1: N 2$

$x b 1(q)=(q-1) * L / N 2 ;$

$z b 1(q)=0$;

$\operatorname{xb} 2(q)=q * L / N 2 ;$

$z b 2(q)=0$;

end

$\%$ Left conductor of dual stripline segment coordinates

for $q=1: N 3$

121 


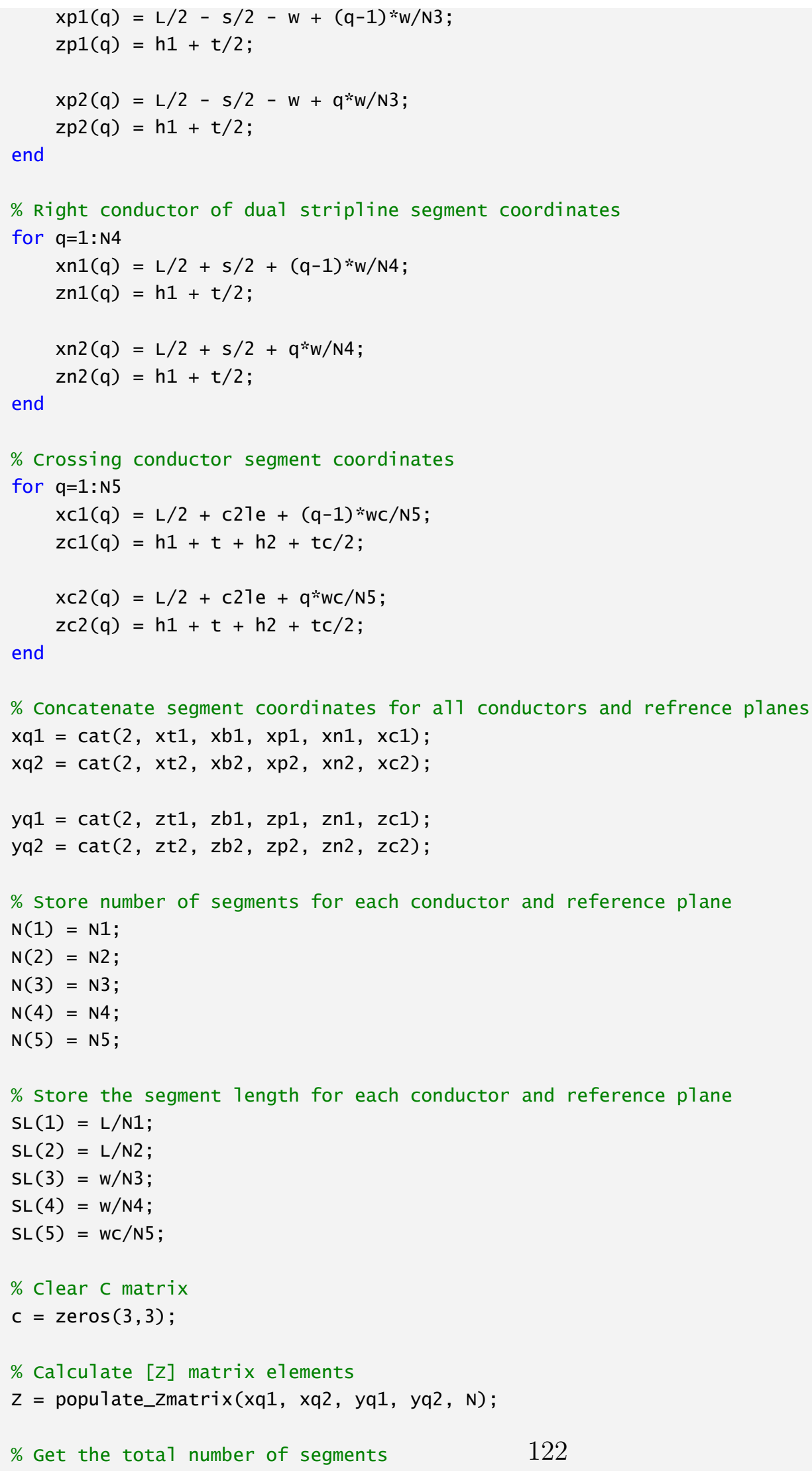




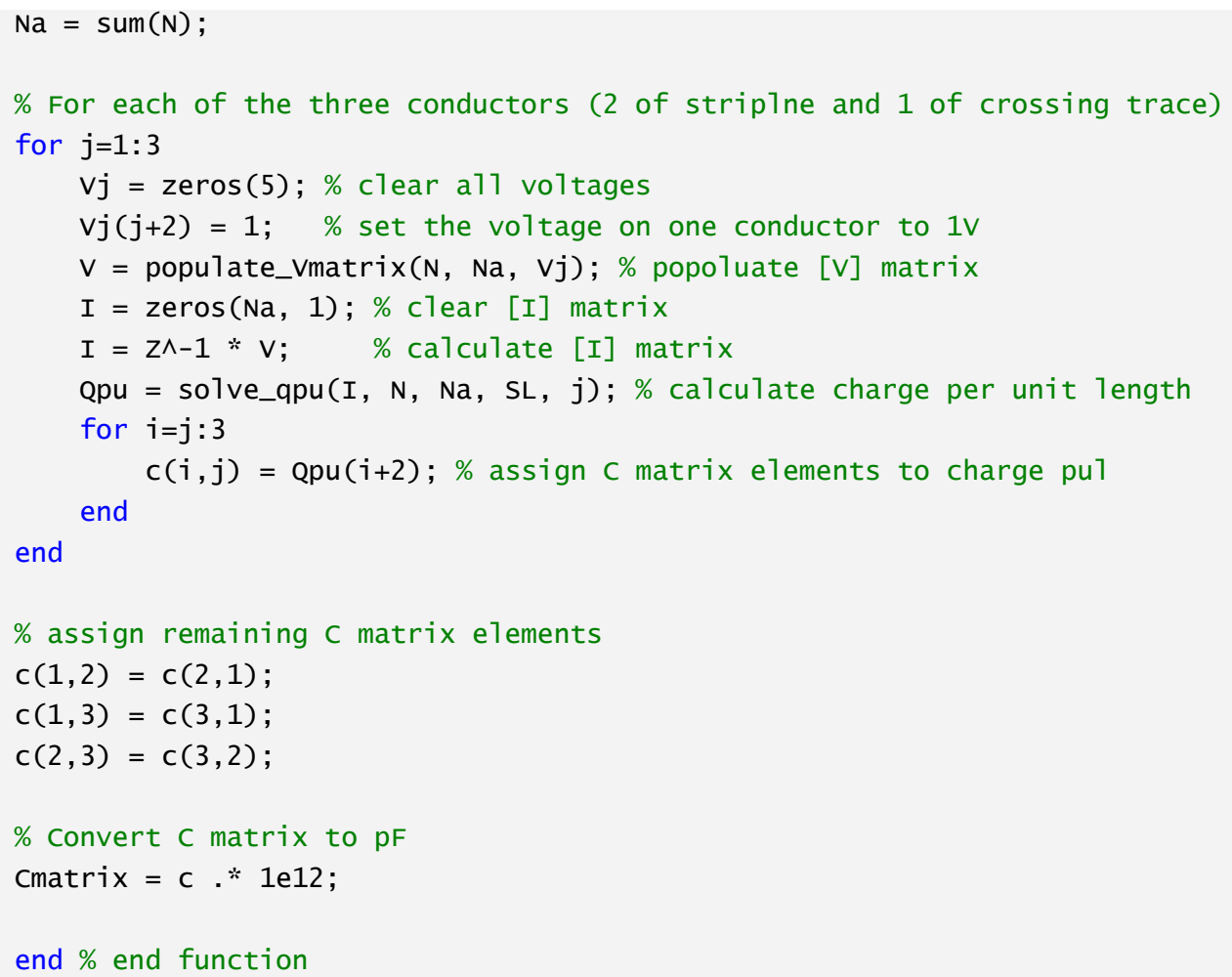

Published with MATLAB ${ }^{\circledR}$ R2019b 


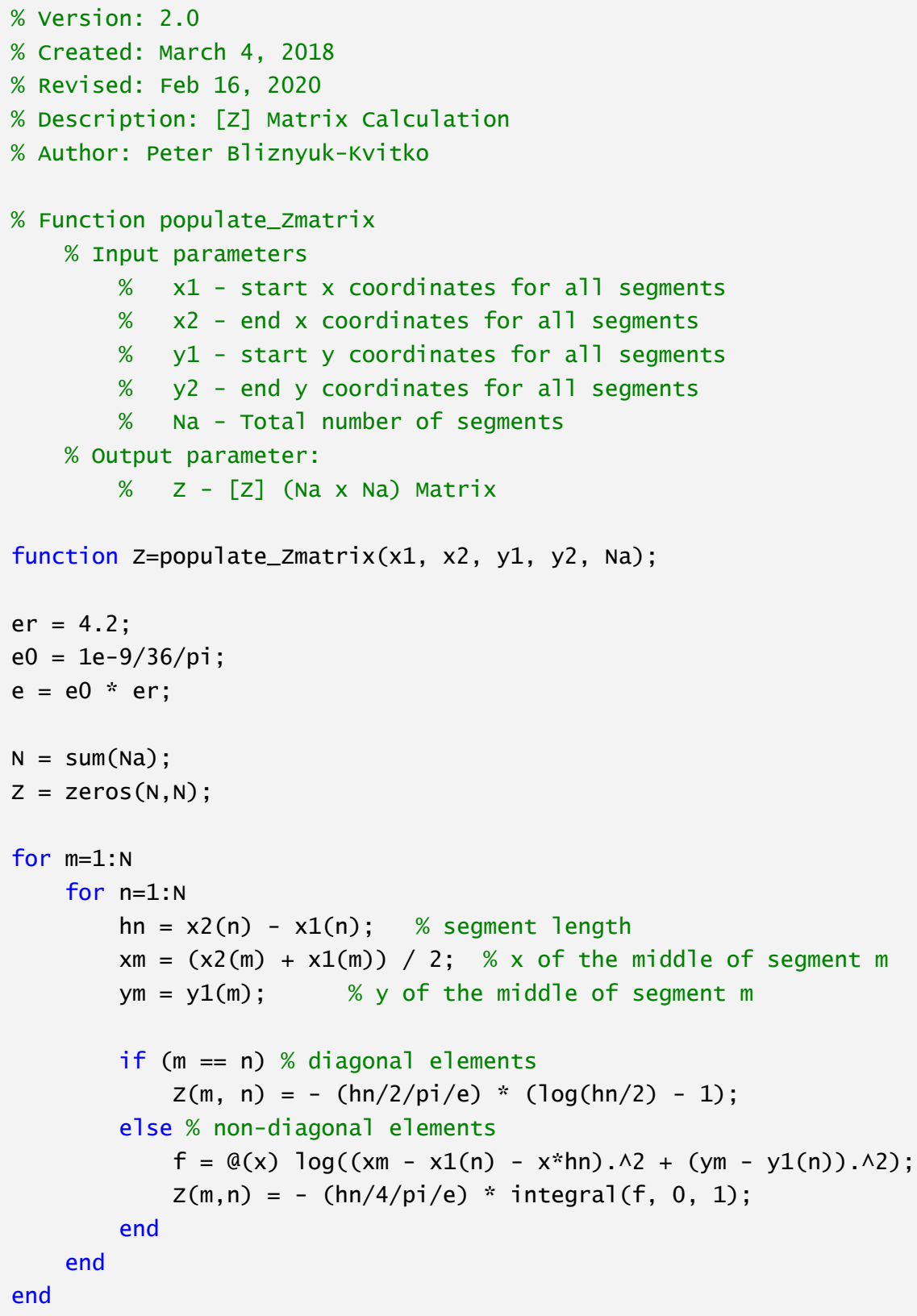

Published with MATLAB ${ }^{\circledR}$ R2019b 


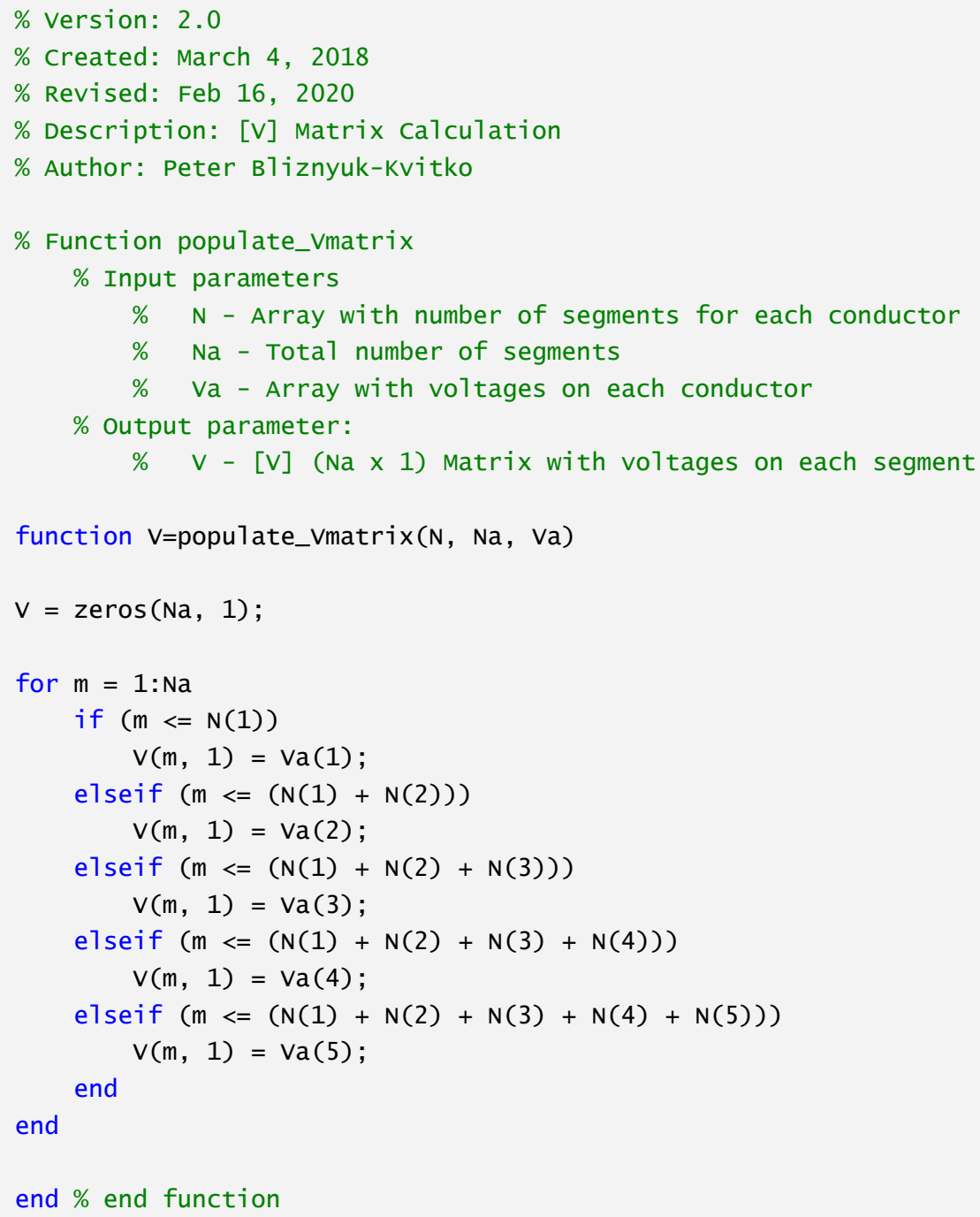

Published with MATLAB ${ }^{\circledR}$ R2019b 
\% version: 2.0

\% created: March 4, 2018

\% Revised: Feb 16, 2020

\% Description: Charge per unit length Calculation

\% Author: Peter Bliznyuk-Kvitko

\% Function solve_qpu

$\%$ Input parameters

$\%$ Io - [I] matrix with an coefficients

$\% \quad \mathrm{~N}$ - Array with number of segments for each conductor

$\% \quad \mathrm{Na}$ - Total number of segments

$\% \quad \mathrm{SL}$ - Array with segment lengths for each conductor

$\% \quad j-$ column of the capacitance matrix

$\%$ output parameter:

\% Q - Array with charge per unit 1ength for each conductor

function Q=solve_qpu(Io, N, Na, SL, j);

$\mathrm{Q}=\operatorname{zeros}(5,1) ;$

if $(j==1)$

$\mathrm{Q}(3,1)=\operatorname{sum}(\operatorname{Io}(\mathrm{N}(1)+\mathrm{N}(2)+1: \mathrm{Na}-\mathrm{N}(5)-\mathrm{N}(4)) * \mathrm{SL}(3)) ;$

$\mathrm{Q}(4,1)=\operatorname{sum}(\operatorname{Io}(\mathrm{N}(1)+\mathrm{N}(2)+\mathrm{N}(3)+1: \mathrm{Na}-\mathrm{N}(5)) * \mathrm{SL}(4)) ;$

$\mathrm{Q}(5,1)=\operatorname{sum}(\operatorname{Io}(\mathrm{N}(1)+\mathrm{N}(2)+\mathrm{N}(3)+\mathrm{N}(4)+1: N a) * \mathrm{SL}(5)) ;$

elseif $(j==2)$

$\mathrm{Q}(4,1)=\operatorname{sum}(\operatorname{Io}(\mathrm{N}(1)+\mathrm{N}(2)+\mathrm{N}(3)+1: \mathrm{Na}-\mathrm{N}(5)) * \mathrm{SL}(4)) ;$

$Q(5,1)=\operatorname{sum}(\operatorname{Io}(N(1)+N(2)+N(3)+N(4)+1: N a) * S L(5))$;

e1se

$Q(5,1)=\operatorname{sum}(\operatorname{Io}(N(1)+N(2)+N(3)+N(4)+1: N a) * S L(5)) ;$

end

Published with MATLAB ${ }^{\circledR}$ R2019b 
Appendix B

Matlab Code for Calculation of

Characteristic Impedance for

Lossless Case 


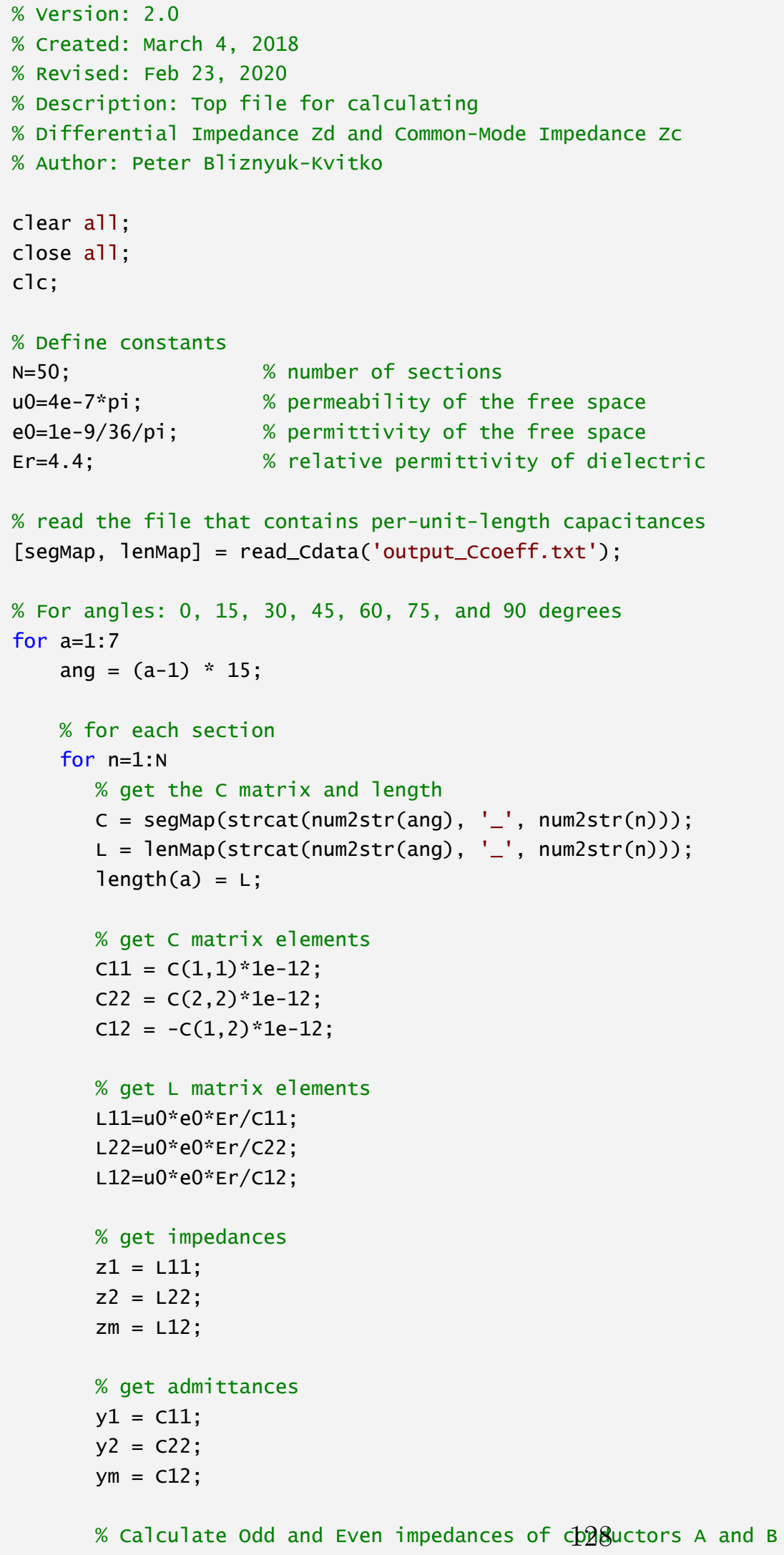




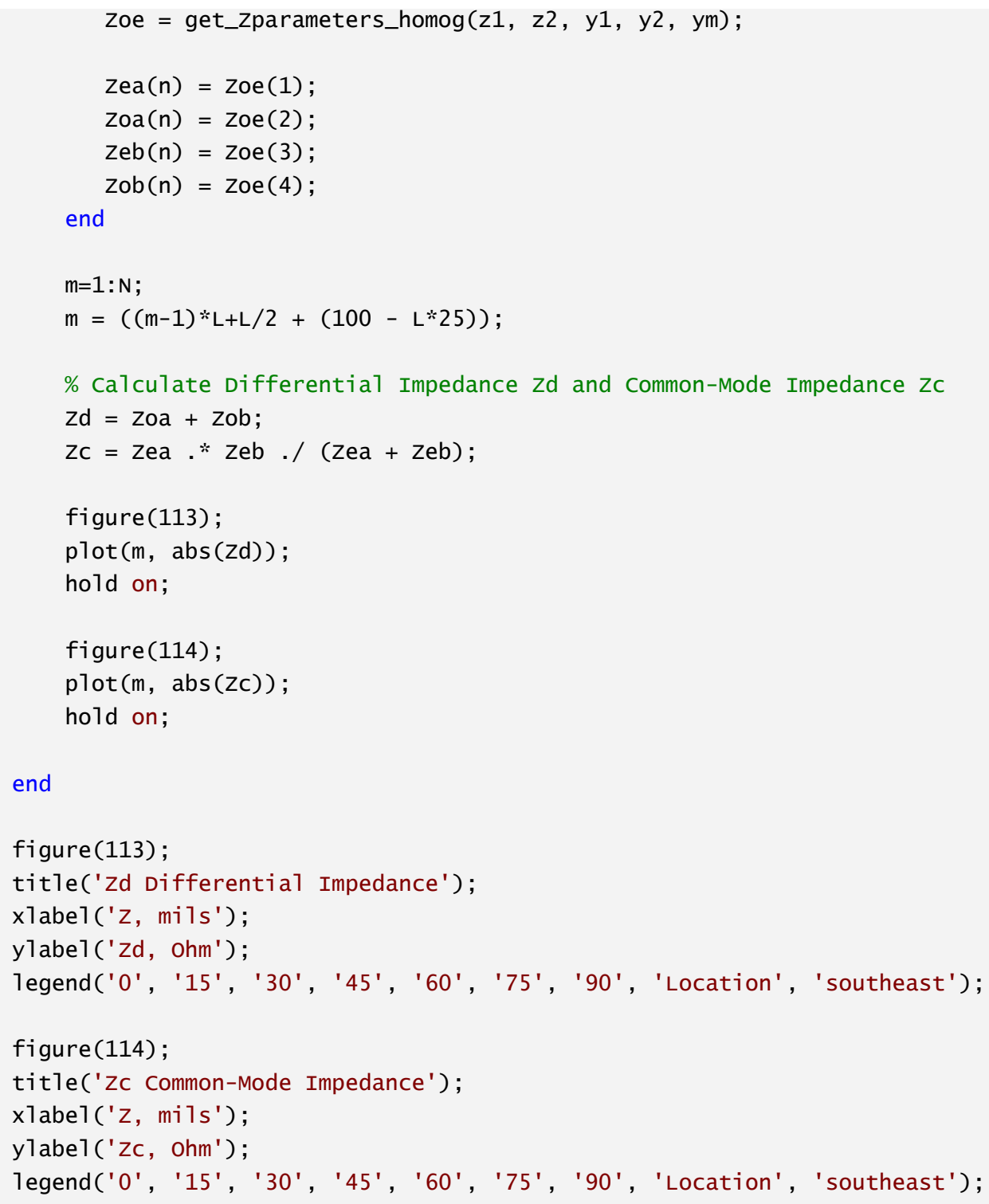

Published with MATLAB ${ }^{\circledR}$ R2019b 


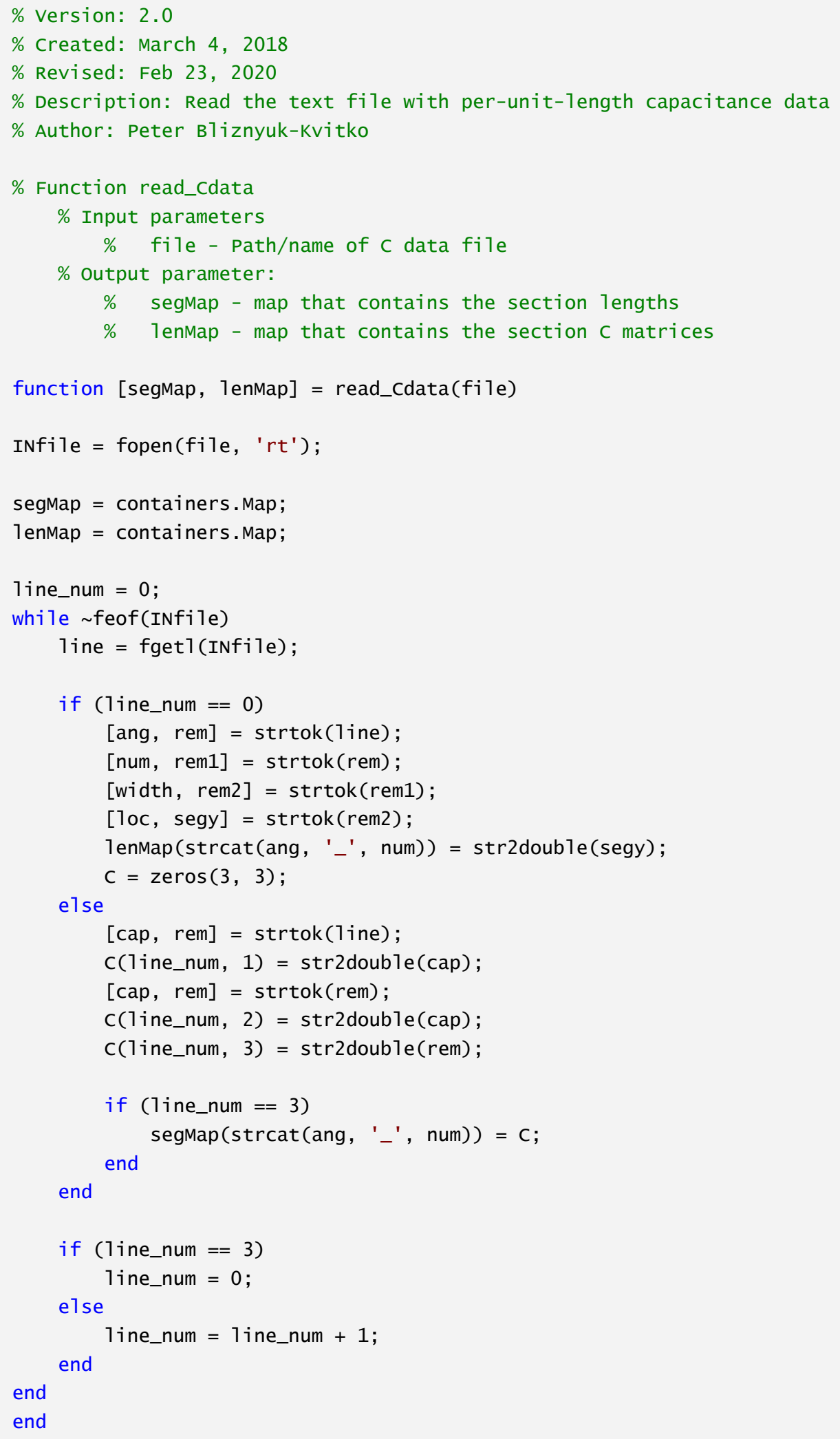

Published with MATLAB ${ }^{\circledR}$ R2019b 


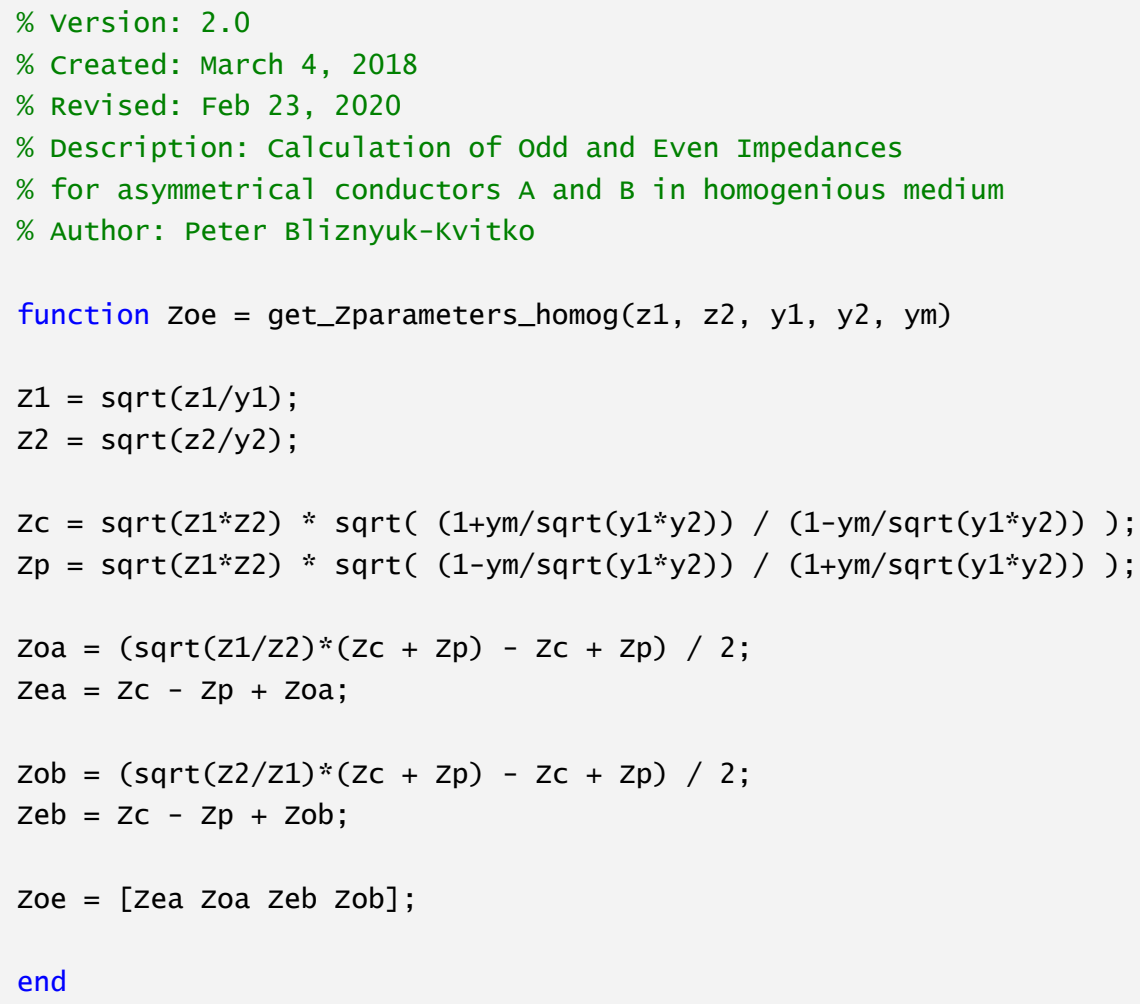

Published with MATLAB ${ }^{\circledR}$ R2019b 
Appendix C

\section{Matlab Code for Calculation of \\ $Z$ parameters}


\% version: 2.0

\% created: March 4, 2018

\% Revised: March 3, 2020

\% Description: $\mathrm{Z}$ parameter calculation for $\mathrm{N}$ crossings

$\%$ at specified angle

\% Author: Peter Bliznyuk-Kvitko

clear a11;

close a11;

clc;

$\mathrm{u} 0=4 \mathrm{e}-7 * \mathrm{pi} ; \quad \%$ permeability of the free space

e0 $=1 \mathrm{e}-9 / 36 / \mathrm{pi} ; \quad \%$ permittivity of the free space

$\mathrm{Er}=4.4$; relative permittivity of dielectric

Ur $=1 ; \quad \%$ magnetic permeability

tand $=0.02 ; \quad \%$ loss tangent

$w d=5 * 2.54 \mathrm{e}-5 ; \quad \%$ width of stripline trace in XY plane, $m$

wdc $=11.313708 * 2.54 \mathrm{e}-5 ; \%$ width of crossing trace in $\mathrm{XY}$ plane, $\mathrm{m}$

th $=0.1 * 2.54 \mathrm{e}-5 ; \%$ thickness of the trace, $m$

Len $=2.941564 * 2.54 \mathrm{e}-5 ; \%$ length of one section in $z$ direction

$\mathrm{nc}=3 ; \quad \%$ number of conductors

$\mathrm{N}=51 ; \quad \%$ number of 45 degree trace crossings

Ns $=50 ; \quad \%$ number of sections

$\mathrm{Nf}=1501 ; \quad \%$ number of frequency points

$f=1$ ogspace $(8.0,10.0, \mathrm{Nf}) ; \%$ frequency range from $100 \mathrm{MHz}$ to $10 \mathrm{GHz}$

$\mathrm{w}=2 * \mathrm{pi} * \mathrm{f} ; \quad \%$ angular frequency

e $=$ Er*e0; $\quad \%$ electrical permittivity of dielectric

$u=u r * u 0 ; \quad \%$ magnetic permeability of dielectric

sigma $=\mathrm{w} * \mathrm{e} *$ tand; $\%$ effective conductivity

ang $=45 ; \quad \%$ crossing trace angle

\% Read the Capacitance Matrices for a11 the sections

[segmap, 1enmap] = read_cdata ('output_ccoeff.txt');

for $i=1: N f \%$ for each frequency point

for $n=1:$ Ns \% for each section

\% Get C Matrix for the 45 degree ang1e, section \# 51-n

$C=\operatorname{segMap}(\operatorname{strcat}($ num2str(ang), '_', num2str(51-n)));

\% Convert capacitance from pF to Farads

$\mathrm{c}=\mathrm{C} * 1 \mathrm{e}-12$;

\% Calculate PUL Inductance Matrix

$\mathrm{L}=\mathrm{u} * \mathrm{e} * \mathrm{C} \wedge-1$

$\%$ find matrix $\mathrm{U}$ whose columns are eigenvectors of $\mathrm{C}$

$\%$ and thetta_sq whose values are eigenva3Bes of $C$ 


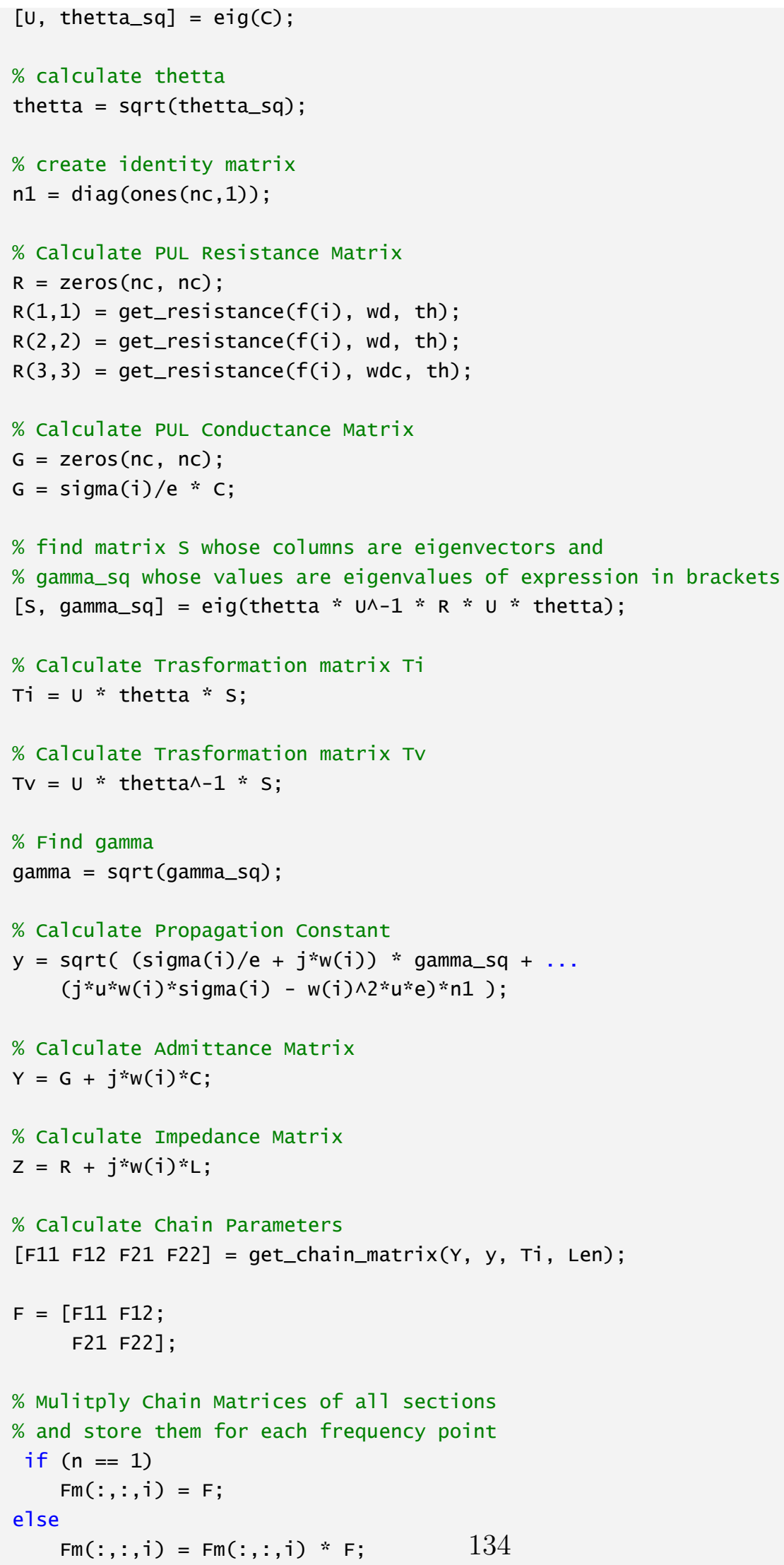




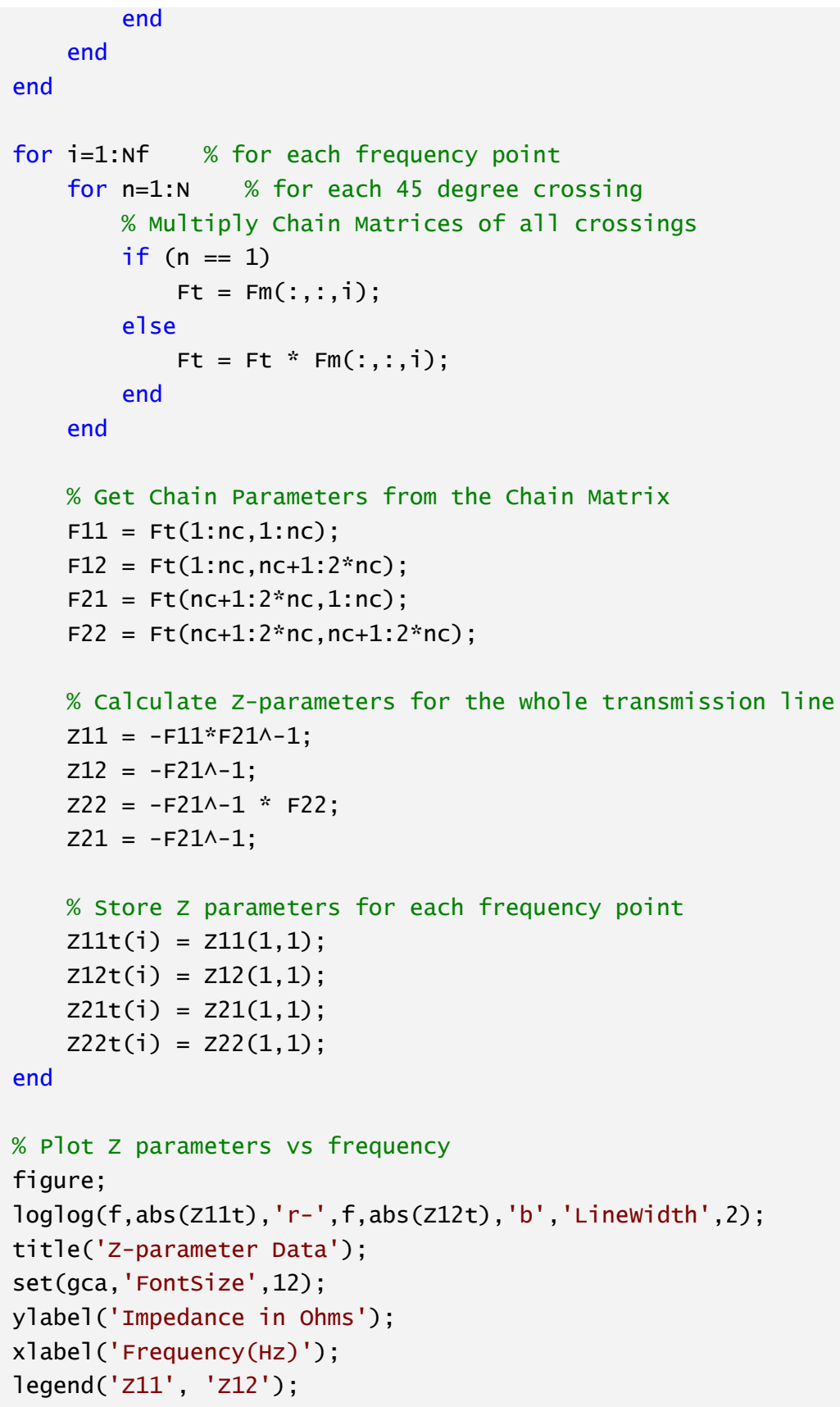

Published with MATLAB ${ }^{\circledR}$ R2019b 


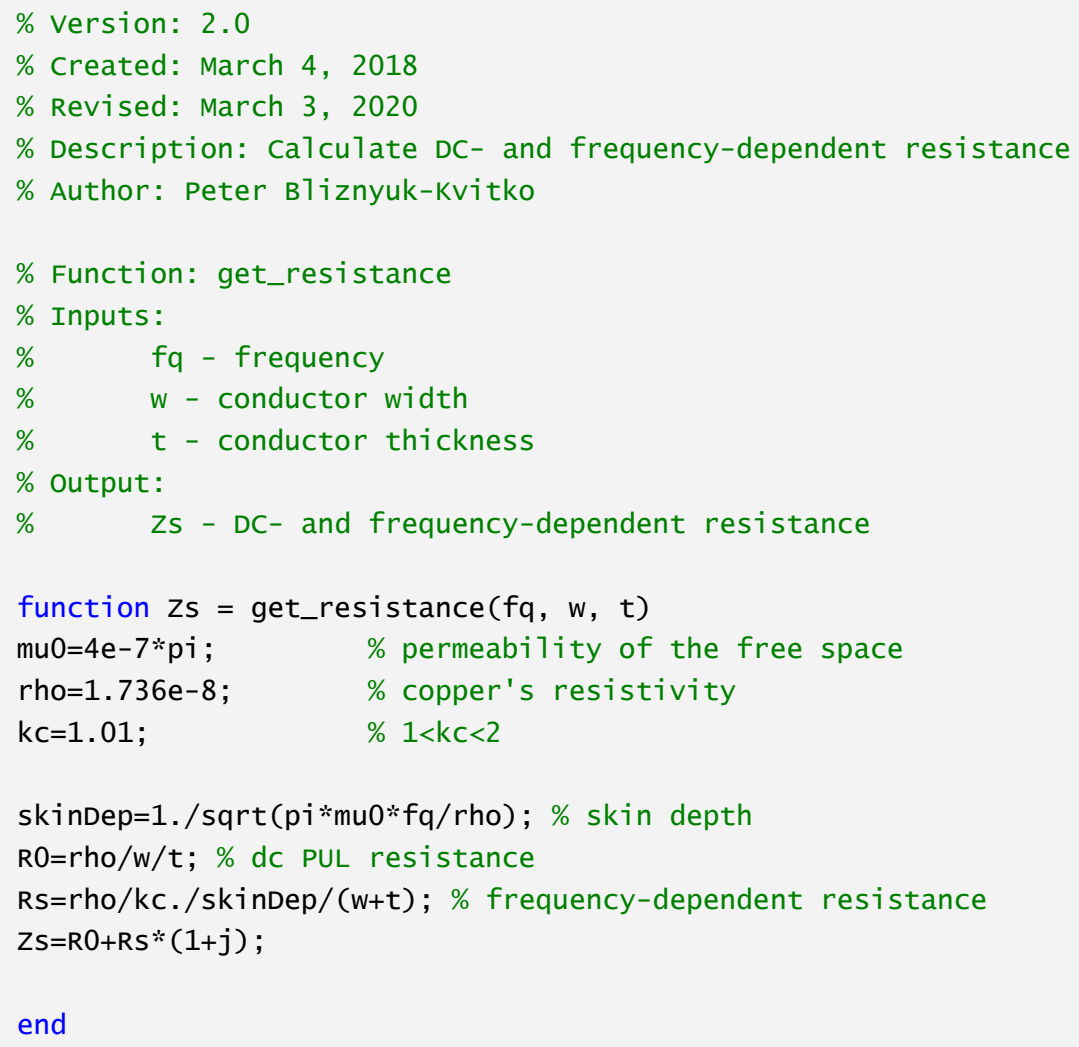

Published with MATLAB ${ }^{\circledR}$ R2019b 


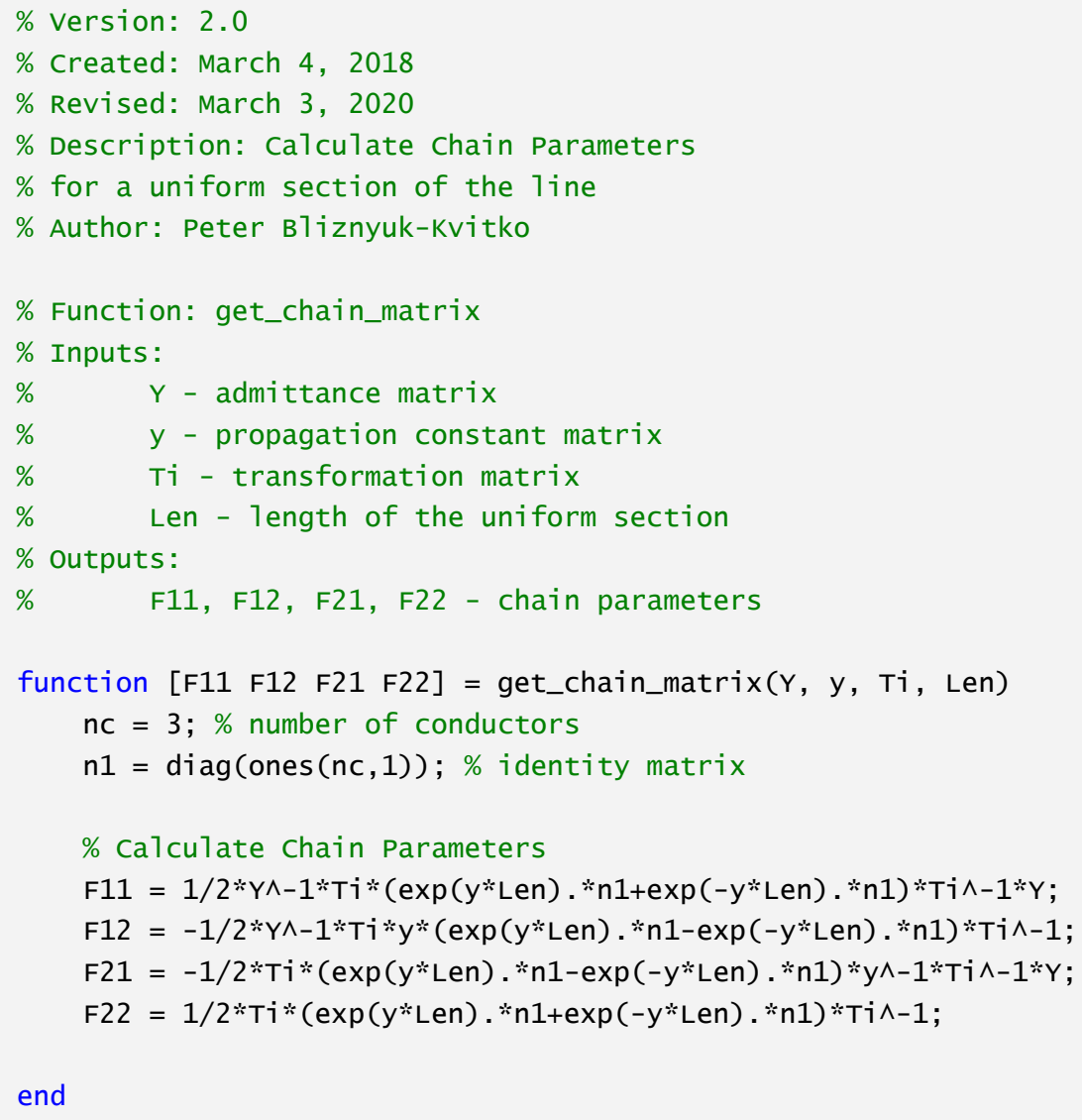

Published with MATLAB ${ }^{\circledR}$ R2019b 